Supporting information

\title{
Stereoselective Preparation of Distant Stereocenters \\ $(1,5)$ within Acyclic Molecules
}

\author{
Anthony Cohen, Jean Chagneau and Ilan Marek* \\ Schulich Faculty of Chemistry, Technion-Israel Institute of Technology, \\ Technion City, Haifa, 32000 (Israel)
}

E-mail: chilanm@technion.ac.il 


\section{Table of Contents}

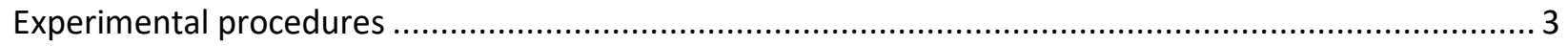

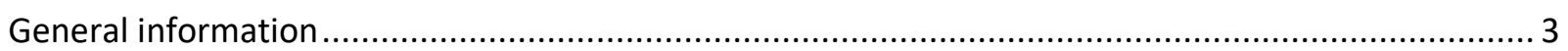

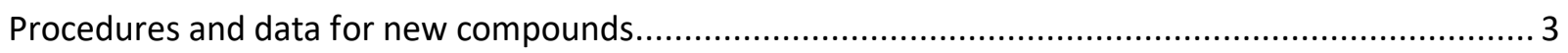

Procedures for the preparation of cyclopropenes .................................................................... 3

Procedures for the preparation of alkenyl halides................................................................ 4

Procedure for the vinylmetalation of cyclopropenes (Scheme 2) ............................................. 4

General procedure for the tandem Heck arylation - ring opening with arylboronic acids (Scheme 3) 8

Procedure for the preparation of alkenyltriflates .................................................................. 15

General procedure for the tandem Heck alkenylation - ring opening with alkenyltriflates (Scheme 4)

Procedure for the Hydrogenation of skipped diene $\mathbf{3 m}$......................................................... 19

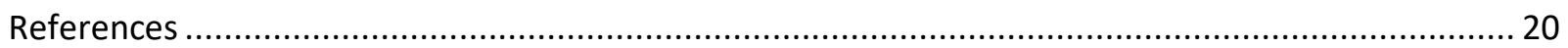

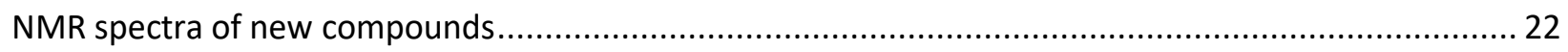




\section{Experimental procedures}

\section{General information}

Unless stated otherwise, reactions were conducted in flame-dried glassware under a positive pressure of argon. Ether and THF were dried from Pure-Solv ${ }^{\circledR}$ Purification System (Innovative Technology@). $\left[\mathrm{Pd}(\mathrm{OTS})_{2}(\mathrm{MeCN})_{2}\right]$ was freshly prepared and stored under a dry atmosphere. ${ }^{1}$ All other commercially obtained reagents were used as received. Thin-layer chromatography (TLC) was conducted with Merck silica gel 60 F254 pre-coated plates $(0.25 \mathrm{~mm})$, and visualized by exposure to UV light $(254 \mathrm{~nm})$ or stained with anisaldehyde, phosphomolybdic acid, or potassium permanganate solutions. Column chromatography was performed using Fluka silica gel $60 \AA$ (40-63mm, 230-400 mesh). NMR spectra were recorded on Bruker spectrometers (AVIII400) and are reported relative to deuterated solvent signals. Chemical shifts are reported in parts per million (ppm) with respect to the residual solvent signal $\mathrm{CDCl}_{3}$ $\left({ }^{1} \mathrm{H}\right.$ NMR: $\delta=7.26 ;{ }^{13} \mathrm{C}$ NMR: $\left.\delta=77.16\right)$. Peak multiplicities are reported as follows: $s=$ singlet, $b s=$ broad singlet, $d=$ doublet, $t=$ triplet, $d d=$ doublet of doublets, $t d=$ triplet of doublets, $m=$ multiplet. Highresolution mass spectra (HRMS) were obtained by the mass spectrometry facility at the Technion. Reactions were monitored by gas chromatography spectrometry (GC) using an Agilent Technologies 7820A GC with an Agilent Technologies 19091J-413 (30 m ×0.3 mm) column. Enantiomeric excesses were determined by chiral HPLC using Agilent $\bigodot 1100$ Series line and CHIRALPAK ${ }^{\circledR} A Y-H(0.46 \mathrm{~cm} \emptyset \times 25 \mathrm{~cm})$ and CHIRALPAK $^{\circledR}$ AZ-H $(0.46 \mathrm{~cm} \emptyset \times 25 \mathrm{~cm})$.

\section{Procedures and data for new compounds}

\section{Procedures for the preparation of cyclopropenes}

Cyclopropenyl carbinols were prepared through the rhodium-catalyzed decomposition of diazoesters in the presence of terminal alkynes, followed by desilylation and reduction of the ester using DIBAL. All cyclopropenes ${ }^{2,3,4}$ were synthesized according to previously reported protocols. The spectral data of all known compounds $(I-a, I-b, I-c)$ were in good agreement with literature reports.
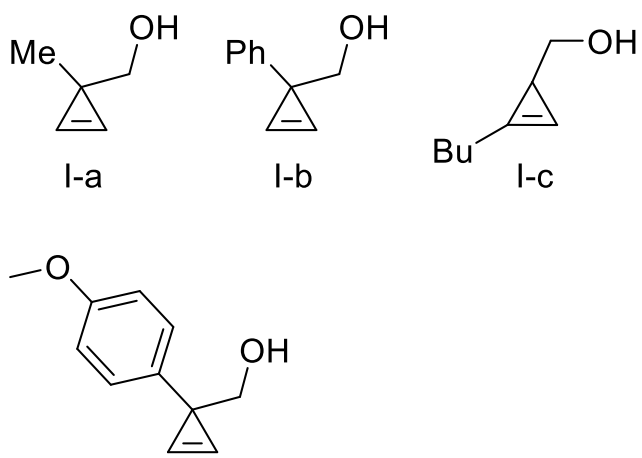

l-d 
(1-(4-methoxyphenyl)cycloprop-2-en-1-yl)methanol. ${ }^{1} \mathrm{H}$ NMR $\left(400 \mathrm{MHz}, \mathrm{CDCl}_{3}\right) \delta 7.35(\mathrm{~s}, 1 \mathrm{H}), 7.15(\mathrm{~d}, \mathrm{~J}=$ $8.8 \mathrm{~Hz}, 1 \mathrm{H}), 6.85(\mathrm{~d}, \mathrm{~J}=8.8 \mathrm{~Hz}, 1 \mathrm{H}), 4.04(\mathrm{~s}, 1 \mathrm{H}), 3.79(\mathrm{~s}, 2 \mathrm{H}) .{ }^{13} \mathrm{C} \mathrm{NMR}\left(101 \mathrm{MHz}, \mathrm{CDCl}_{3}\right) \delta$ 157.9, 137.9, $130.3,127.4,113.9,113.8,113.7,68.2,55.4,28.7,11.1$. HRMS m/z: (APCI) $[\mathrm{M}+\mathrm{H}]^{+}$, calculated for $\mathrm{C}_{11} \mathrm{H}_{13} \mathrm{O}_{2}$; 177.0896; found 177.0910 .

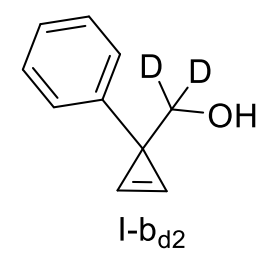

(1-phenylcycloprop-2-en-1-yl)methan-1d2-ol. ${ }^{2}{ }^{1} \mathrm{H}$ NMR $\left(400 \mathrm{MHz}, \mathrm{CDCl}_{3}\right) \delta 7.24-7.15(\mathrm{~m}, 1 \mathrm{H}), 7.14-$ 7.05 (m, 1H). ${ }^{13} \mathrm{C} \mathrm{NMR}\left(101 \mathrm{MHz}, \mathrm{CDCl}_{3}\right) \delta$ 145.9, 128.3, 126.4, 125.9, 113.0. HRMS m/z: (APCI) [M-OH] ${ }^{+}$, calculated for $\mathrm{C}_{10} \mathrm{H}_{7} \mathrm{D}_{2} ; 131.0824$; found 131.0836 .

\section{Procedures for the preparation of alkenyl halides}

$(Z)$ - and (E)-1-bromoprop-1-ene were used as received from commercial sources. (Z)-1-iodohex-1-ene, ${ }^{5}$ $(E)$-1-bromohex-1-ene, ${ }^{6}(E)$-1-iodo-2-methylhex-1-ene ${ }^{7}$ were prepared following literature reports and analytic data were in full agreement with the reports.

\section{Procedure for the vinylmetalation of cyclopropenes (Scheme 2)}

In a flamed-dried three-neck flask under argon atmosphere, alkenyl halide (1 equiv, scale of 2-20 mmol) was dissolved in dry $\mathrm{Et}_{2} \mathrm{O}(0.2 \mathrm{M})$. The solution was cooled to $-78^{\circ} \mathrm{C}$ using an acetone/dry ice cooling bath, and tert-BuLi (1.7 M, 2 equiv) was added dropwise while maintaining the temperature below $-60^{\circ} \mathrm{C}$. The cooling bath was then removed and the solution was let to warm up to $0{ }^{\circ} \mathrm{C}$. The cooling bath was then replaced and upon reaching a temperature of $-40{ }^{\circ} \mathrm{C}$, copper iodide $(10 \mathrm{~mol} \%)$ was added to the reaction mixture and was stirred for 5 minutes. A solution of cyclopropene carbinol in $\mathrm{Et}_{2} \mathrm{O}(1 \mathrm{M}, 0.5$ equiv, scale of 1-10 mmol) was then added and the solution was warmed up to $0{ }^{\circ} \mathrm{C}$ over a period of one hour by placing an ice bath. Following the complete conversion of the cyclopropene (conversion monitored by TLC and/or GC), the reaction was quenched with an aqueous saturated solution of ammonium chloride and the aqueous phase was extracted with $\mathrm{Et}_{2} \mathrm{O}$ three times. The combined organic phases were dried over anhydrous $\mathrm{MgSO}_{4}$ and concentrated under reduced pressure. The resulting crude mixture was purified by column chromatography.<smiles>C/C=C\[C@H]1C[C@@]1(CO)c1ccccc1</smiles>

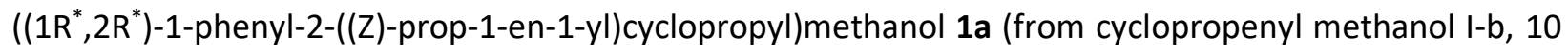
mmol, $72 \%$ yield): ${ }^{1} \mathrm{H}$ NMR $\left(400 \mathrm{MHz}, \mathrm{CDCl}_{3}\right) \delta 7.34-7.30(\mathrm{~m}, 2 \mathrm{H}), 7.26$ (dd, J = 10.3, $4.9 \mathrm{~Hz}, 2 \mathrm{H}$ ), 7.16 (dd, $\mathrm{J}=10.8,4.6 \mathrm{~Hz}, 1 \mathrm{H}), 5.65-5.56(\mathrm{~m}, 1 \mathrm{H}), 5.27(\mathrm{ddd}, \mathrm{J}=10.6,9.0,1.7 \mathrm{~Hz}, 1 \mathrm{H}), 3.84(\mathrm{~d}, \mathrm{~J}=11.9 \mathrm{~Hz}, 1 \mathrm{H}), 3.67$ 
(d, J = 11.9 Hz, 1H), $1.95(\mathrm{dd}, \mathrm{J}=14.7,8.6 \mathrm{~Hz}, 1 \mathrm{H}), 1.73(\mathrm{dd}, \mathrm{J}=6.8,1.6 \mathrm{~Hz}, 3 \mathrm{H}), 1.31$ (dd, J = 8.5, $4.9 \mathrm{~Hz}$, $1 \mathrm{H}), 1.18(\mathrm{~s}, 1 \mathrm{H}), 0.91-0.88(\mathrm{~m}, 1 \mathrm{H}) .{ }^{13} \mathrm{C} N M R\left(101 \mathrm{MHz}, \mathrm{CDCl}_{3}\right) \delta 143.8,128.8,128.8,128.5,127.1,126.7$, 67.8, 34.6, 23.0, 20.3, 13.4. HRMS m/z: (APCI) $[\mathrm{M}+\mathrm{H}]^{+}$, calculated for $\mathrm{C}_{13} \mathrm{H}_{17} \mathrm{O} ; 189.1274$; found 189.1277.<smiles>[2H][C@@]1([13C]([2H])([2H])O)C[C@@H]1/C=C\C</smiles>

$\left(\left(1 R^{*}, 2 R^{*}\right)-1-p h e n y l-2-((Z)-p r o p-1-e n-1-y l) c y c l o p r o p y l\right) m e t h a n-d 2-o l ~ 1 a_{d 2}$ (from cyclopropenyl methanol I$\mathrm{b}_{\mathrm{d} 2}, 1 \mathrm{mmol}, 75 \%$ yield): ${ }^{1} \mathrm{H}$ NMR $\left(400 \mathrm{MHz}, \mathrm{CDCl}_{3}\right) \delta 7.32$ (d, J = 8.0 Hz, 2H), 7.25 (t, J = 7.4 Hz, 2H), $7.19-$ $7.14(\mathrm{~m}, 1 \mathrm{H}), 5.60(\mathrm{dq}, \mathrm{J}=13.6,6.8 \mathrm{~Hz}, 1 \mathrm{H}), 5.26(\mathrm{t}, \mathrm{J}=9.8 \mathrm{~Hz}, 1 \mathrm{H}), 1.95(\mathrm{dd}, \mathrm{J}=14.8,8.5 \mathrm{~Hz}, 1 \mathrm{H}), 1.73(\mathrm{~d}$, $\mathrm{J}=6.8 \mathrm{~Hz}, 3 \mathrm{H}), 1.41(\mathrm{~s}, 1 \mathrm{H}), 1.31(\mathrm{dd}, \mathrm{J}=8.5,4.9 \mathrm{~Hz}, 1 \mathrm{H}), 0.89(\mathrm{t}, \mathrm{J}=5.3 \mathrm{~Hz}, 1 \mathrm{H}) .{ }^{13} \mathrm{C} \mathrm{NMR}\left(101 \mathrm{MHz}, \mathrm{CDCl}_{3}\right)$ $\delta 143.7,128.9,128.7,128.5,127.1,126.7,67.1,34.4,23.0,20.3,13.4 . \mathrm{HRMS} \mathrm{m} / \mathrm{z}$ : (APCl) [M-OH] ${ }^{+}$, calculated for $\mathrm{C}_{13} \mathrm{H}_{13} \mathrm{D}_{2} ; 173.1299$; found 173.1292 .<smiles>C/C=C/[C@@H]1C[C@@]1(CO)c1ccccc1</smiles>

((1R* $\left.2 R^{*}\right)$-1-phenyl-2-((E)-prop-1-en-1-yl)cyclopropyl)methanol 1b (from cyclopropenyl methanol I-b, 5 mmol, 75\% yield): ${ }^{1} \mathrm{H}$ NMR $\left(400 \mathrm{MHz}, \mathrm{CDCl}_{3}\right) \delta 7.29-7.19(\mathrm{~m}, 4 \mathrm{H}), 7.15-7.10(\mathrm{~m}, 1 \mathrm{H}), 5.66-5.57(\mathrm{~m}$, $1 \mathrm{H}), 5.39$ (ddd, J = 15.2, 7.8, 1.5 Hz, $1 \mathrm{H}$ ), $3.78(\mathrm{dd}, \mathrm{J}=11.7,4.6 \mathrm{~Hz}, 1 \mathrm{H}), 3.63(\mathrm{~d}, \mathrm{~J}=11.7 \mathrm{~Hz}, 1 \mathrm{H}), 1.76$ (dd, $\mathrm{J}=14.4,8.0 \mathrm{~Hz}, 1 \mathrm{H}), 1.65(\mathrm{dd}, \mathrm{J}=6.5,1.0 \mathrm{~Hz}, 3 \mathrm{H}), 1.16(\mathrm{dd}, \mathrm{J}=8.5,5.0 \mathrm{~Hz}, 1 \mathrm{H}), 0.89-0.84(\mathrm{~m}, 1 \mathrm{H}) .{ }^{13} \mathrm{C}$ $\operatorname{NMR}\left(101 \mathrm{MHz}, \mathrm{CDCl}_{3}\right) \delta 143.8,128.9,128.6,128.5,127.5,126.6,67.2,33.9,27.6,18.7,18.2$. HRMS m/z: (APCI) $[\mathrm{M}-\mathrm{OH}]^{+}$, calculated for $\mathrm{C}_{13} \mathrm{H}_{15} ; 171.1168$; found 171.1158 .<smiles>C/C=C\[C@@H]1C[C@]1(C)CO</smiles>

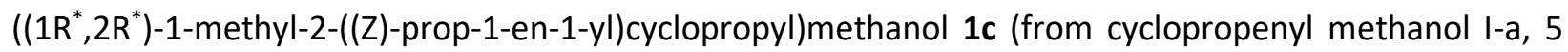
mmol, 76\% yield): ${ }^{1} \mathrm{H}$ NMR $\left(400 \mathrm{MHz}, \mathrm{CDCl}_{3}\right) \delta 5.59-5.50(\mathrm{~m}, 1 \mathrm{H}), 5.20-5.13(\mathrm{~m}, 1 \mathrm{H}), 3.65(\mathrm{~d}, \mathrm{~J}=11.6$ $\mathrm{Hz}, 1 \mathrm{H}), 3.43(\mathrm{~d}, \mathrm{~J}=11.6 \mathrm{~Hz}, 1 \mathrm{H}), 1.74(\mathrm{dd}, \mathrm{J}=6.8,1.7 \mathrm{~Hz}, 3 \mathrm{H}), 1.49(\mathrm{td}, \mathrm{J}=8.9,5.7 \mathrm{~Hz}, 1 \mathrm{H}), 1.22(\mathrm{~s}, 3 \mathrm{H})$, $0.81(\mathrm{dd}, \mathrm{J}=8.2,4.6 \mathrm{~Hz}, 1 \mathrm{H}), 0.53(\mathrm{t}, \mathrm{J}=5.0 \mathrm{~Hz}, 1 \mathrm{H}) .{ }^{13} \mathrm{C} \mathrm{NMR}\left(101 \mathrm{MHz}, \mathrm{CDCl}_{3}\right) \delta$ 129.6, 125.9, 68.1, 65.9, $34.2,25.3,22.4,22.2,22.2,20.6,15.3,14.1,13.3$. HRMS m/z: (APCl) $[\mathrm{M}-\mathrm{OH}]^{+}$, calculated for $\mathrm{C}_{8} \mathrm{H}_{13}$; 109.1012; found 109.0989 .<smiles>C/C=C/[C@@H]1C[C@@]1(C)CO</smiles>

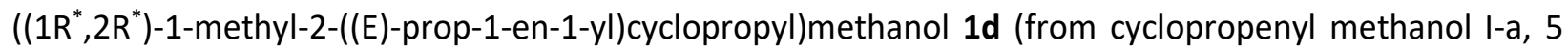
mmol, 66\% yield): ${ }^{1} \mathrm{H}$ NMR (400 MHz, $\left.\mathrm{CDCl}_{3}\right) \delta 5.41(\mathrm{dq}, \mathrm{J}=12.9,6.5 \mathrm{~Hz}, 1 \mathrm{H}), 5.14-5.05(\mathrm{~m}, 1 \mathrm{H}$ ), 3.45 (dd, $\mathrm{J}=11.5,8.0 \mathrm{~Hz}, 1 \mathrm{H}), 3.26-3.22(\mathrm{~m}, 1 \mathrm{H}), 1.49(\mathrm{dd}, \mathrm{J}=6.5,1.5 \mathrm{~Hz}, 3 \mathrm{H}), 0.99(\mathrm{~s}, 3 \mathrm{H}), 0.49(\mathrm{dd}, \mathrm{J}=8.2,4.8 \mathrm{~Hz}$, $1 \mathrm{H}), 0.33(\mathrm{t}, \mathrm{J}=5.1 \mathrm{~Hz}, 1 \mathrm{H}) .{ }^{13} \mathrm{C}$ NMR $\left(101 \mathrm{MHz}, \mathrm{CDCl}_{3}\right) \delta 129.6,126.4,67.6,65.9,34.2,30.3,26.7,24.9$, 
22.4, 22.1, 19.7, 18.8, 18.1, 15.3, 14.1. HRMS m/z: (APCI) [M-OH] $]^{+}$, calculated for $\mathrm{C}_{8} \mathrm{H}_{13}$; 109.1012; found 109.0989 .<smiles>OC[C@]1([C@@H]2C[C@@H]2Br)C[C@@H]1Br</smiles>

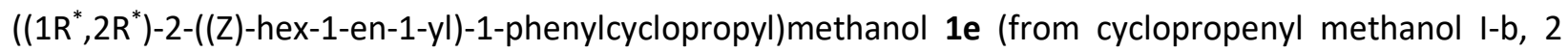
mmol, 68\% yield): ${ }^{1} \mathrm{H}$ NMR $\left(400 \mathrm{MHz}, \mathrm{CDCl}_{3}\right) \delta 7.32-7.28(\mathrm{~m}, 2 \mathrm{H}), 7.25(\mathrm{dd}, \mathrm{J}=10.2,4.9 \mathrm{~Hz}, 2 \mathrm{H}), 7.18-$ $7.12(\mathrm{~m}, 1 \mathrm{H}), 5.51(\mathrm{dtd}, \mathrm{J}=10.7,7.3,0.8 \mathrm{~Hz}, 1 \mathrm{H}), 5.22(\mathrm{dd}, \mathrm{J}=10.7,9.2 \mathrm{~Hz}, 1 \mathrm{H}), 3.80(\mathrm{~d}, \mathrm{~J}=11.9 \mathrm{~Hz}, 1 \mathrm{H})$, $3.67(\mathrm{~d}, \mathrm{~J}=11.9 \mathrm{~Hz}, 1 \mathrm{H}), 2.15(\mathrm{q}, \mathrm{J}=7.2 \mathrm{~Hz}, 2 \mathrm{H}), 1.93(\mathrm{td}, \mathrm{J}=8.8,6.4 \mathrm{~Hz}, 1 \mathrm{H}), 1.31(\mathrm{td}, \mathrm{J}=8.7,3.9 \mathrm{~Hz}, 4 \mathrm{H})$, $0.86(\mathrm{dt}, \mathrm{J}=14.2,6.2 \mathrm{~Hz}, 5 \mathrm{H}) .{ }^{13} \mathrm{C}$ NMR $\left(101 \mathrm{MHz}, \mathrm{CDCl}_{3}\right) \delta 143.8,133.2,128.8,128.5,127.8,126.7,67.8$, 34.6, 31.8, 27.6, 23.2, 22.5, 20.4, 14.1. HRMS m/z: (APCI) $[\mathrm{M}+\mathrm{H}]^{+}$, calculated for $\mathrm{C}_{16} \mathrm{H}_{23} \mathrm{O} ; 231.1743$; found 231.1749 .<smiles>OC[C@]1(P)C[C@@H]1C=CBr</smiles>

((1R,2R)-2-((E)-hex-1-en-1-yl)-1-phenylcyclopropyl)methanol 1f (from cyclopropenyl methanol I-b, 2 mmol, 75\% yield): ${ }^{1} \mathrm{H}$ NMR $\left(400 \mathrm{MHz}, \mathrm{CDCl}_{3}\right) \delta 7.20-7.11(\mathrm{~m}, 4 \mathrm{H}), 7.08-7.01(\mathrm{~m}, 1 \mathrm{H}), 5.56-5.47(\mathrm{~m}$, $1 \mathrm{H}), 5.29(\mathrm{dd}, \mathrm{J}=15.2,7.7 \mathrm{~Hz}, 1 \mathrm{H}), 3.70(\mathrm{~d}, \mathrm{~J}=11.8 \mathrm{~Hz}, 1 \mathrm{H}), 3.56(\mathrm{~d}, \mathrm{~J}=11.8 \mathrm{~Hz}, 1 \mathrm{H}), 1.90(\mathrm{q}, \mathrm{J}=6.7 \mathrm{~Hz}$, $2 \mathrm{H}), 1.68(\mathrm{dd}, \mathrm{J}=14.2,7.9 \mathrm{~Hz}, 1 \mathrm{H}), 1.20-1.16(\mathrm{~m}, 3 \mathrm{H}), 1.10-1.06(\mathrm{~m}, 2 \mathrm{H}), 0.82-0.78(\mathrm{~m}, 1 \mathrm{H}), 0.76-$ $0.72(\mathrm{~m}, 4 \mathrm{H}) .{ }^{13} \mathrm{C}$ NMR $\left(101 \mathrm{MHz}, \mathrm{CDCl}_{3}\right) \delta 143.8,133.3,128.6,128.5,127.6,126.5,67.2,33.9,32.4,31.8$, 27.6, 22.3, 18.8, 14.0. HRMS m/z: (APCI) $[\mathrm{M}+\mathrm{H}]^{+}$, calculated for $\mathrm{C}_{16} \mathrm{H}_{23} \mathrm{O} ; 231.1743$; found 231.1746.

Enantioenriched 1f was prepared according to a known procedure ${ }^{8}$ Its enantiopurity was determined by HPLC: separated on Daicel Chemical CHIRALPAK ${ }^{\circledR} \mathrm{OX}-\mathrm{H}(0.46 \mathrm{~cm} \emptyset \times 25 \mathrm{~cm})$. Separation conditions: eluent 99:1 hexane:isopropanol , flow rate $: 0.5 \mathrm{ml} / \mathrm{min}, 23{ }^{\circ} \mathrm{C} . R_{t}($ major $)=23.155 \mathrm{~min} ; R_{t}($ minor $)=24.382 \mathrm{~min}$.
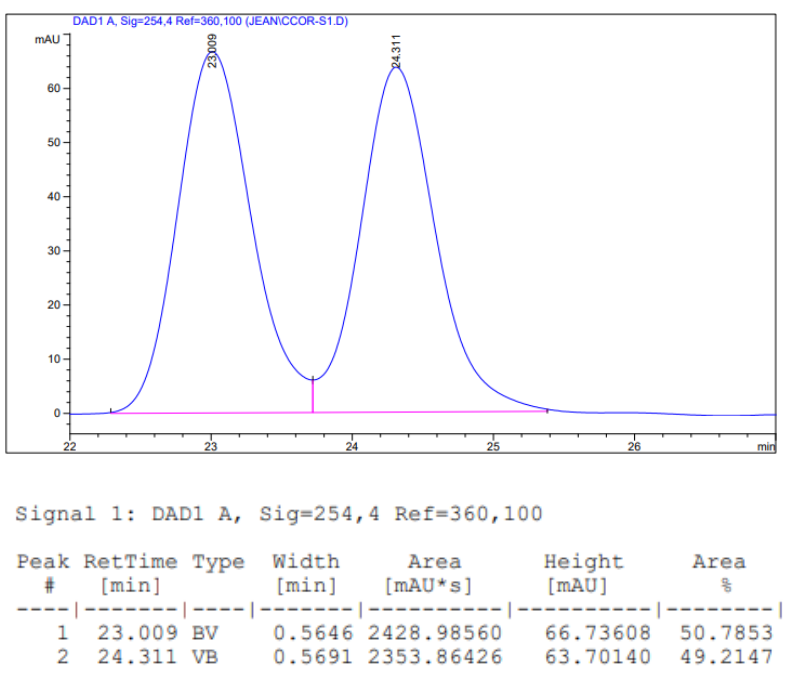


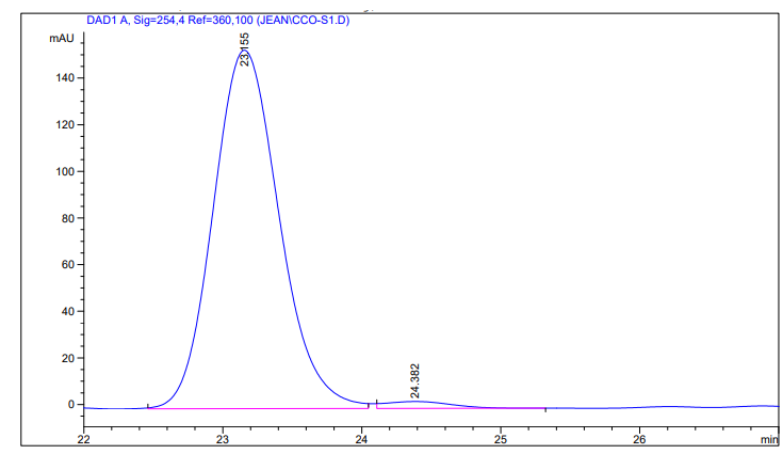

Signal 1: DAD1 A, Sig=254,4 Ref=360,100

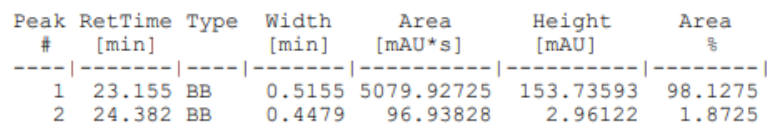<smiles>CC(Br)=C[C@H]1C[C@@]1(CO)c1ccccc1</smiles>

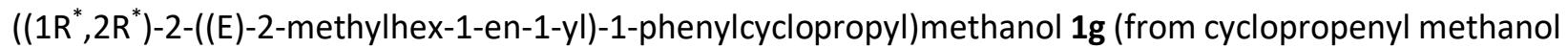
I-b, $2 \mathrm{mmol}, 71 \%$ yield): ${ }^{1} \mathrm{H}$ NMR $\left(400 \mathrm{MHz}, \mathrm{CDCl}_{3}\right) \delta 7.39$ (dd, J = 8.0, $\left.0.9 \mathrm{~Hz}, 2 \mathrm{H}\right), 7.33$ (dd, J = 10.3, 4.9 $\mathrm{Hz}, 2 \mathrm{H}), 7.25-7.20(\mathrm{~m}, 1 \mathrm{H}), 5.09(\mathrm{~d}, \mathrm{~J}=8.2 \mathrm{~Hz}, 1 \mathrm{H}), 3.90(\mathrm{dd}, \mathrm{J}=11.9,8.5 \mathrm{~Hz}, 1 \mathrm{H}), 3.75(\mathrm{dd}, \mathrm{J}=11.9,4.5$ $\mathrm{Hz}, 1 \mathrm{H}), 2.05(\mathrm{t}, \mathrm{J}=7.5 \mathrm{~Hz}, 2 \mathrm{H}), 1.91(\mathrm{dd}, \mathrm{J}=14.4,8.4 \mathrm{~Hz}, 1 \mathrm{H}), 1.79(\mathrm{~s}, 3 \mathrm{H}), 1.36(\mathrm{ddt}, \mathrm{J}=28.2,22.1,7.5 \mathrm{~Hz}$, $6 \mathrm{H}), 0.95-0.89(\mathrm{~m}, 4 \mathrm{H}) .{ }^{13} \mathrm{C} \mathrm{NMR}\left(101 \mathrm{MHz}, \mathrm{CDCl}_{3}\right) \delta 144.0,139.8,128.6,128.5,126.5,122.4,68.0,39.4$, $34.3,30.2,23.9,22.4,20.5,16.8,14.0$. HRMS m/z: (APCI) $[\mathrm{M}-\mathrm{OH}]^{+}$, calculated for $\mathrm{C}_{17} \mathrm{H}_{23} ; 227.1800$; found 227.1811.<smiles>CC(Br)=C[C@H]1C[C@@]1(C)CO</smiles>

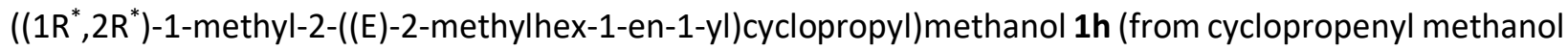
I-a, $1 \mathrm{mmol}, 70 \%$ yield): ${ }^{1} \mathrm{H}$ NMR (400 MHz, $\left.\mathrm{CDCl}_{3}\right) \delta 4.86$ (dd, J = 8.4, $\left.1.2 \mathrm{~Hz}, 1 \mathrm{H}\right), 3.55(\mathrm{~d}, \mathrm{~J}=11.6 \mathrm{~Hz}, 1 \mathrm{H})$, $3.35(\mathrm{~d}, \mathrm{~J}=11.6 \mathrm{~Hz}, 1 \mathrm{H}), 1.94-1.88(\mathrm{~m}, 2 \mathrm{H}), 1.65(\mathrm{~d}, \mathrm{~J}=1.1 \mathrm{~Hz}, 3 \mathrm{H}), 1.34-1.16(\mathrm{~m}, 5 \mathrm{H}), 1.13(\mathrm{~s}, 2 \mathrm{H}), 0.82$ (dd, J = 9.1, 5.3 Hz, 3H), 0.69 (dd, J = 8.2, $4.5 \mathrm{~Hz}, 1 \mathrm{H}), 0.41(\mathrm{t}, \mathrm{J}=5.0 \mathrm{~Hz}, 1 \mathrm{H}) .{ }^{13} \mathrm{C} \mathrm{NMR}\left(101 \mathrm{MHz}, \mathrm{CDCl}_{3}\right) \delta$ $138.46,123.15,68.25,39.30,30.24,24.99,22.97,22.38,22.22,20.45,16.54,14.00$. HRMS m/z: (APCI) [M$\mathrm{OH}]^{+}$, calculated for $\mathrm{C}_{12} \mathrm{H}_{21} ; 165.1643$; found 165.1638 .<smiles>COC/C=C\[C@@H]1C[C@@]1(CO)c1ccc(OC)cc1</smiles>

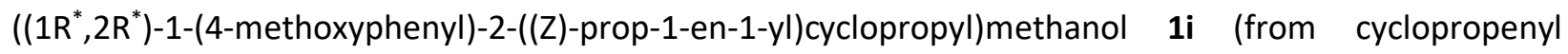
methanol I-d, $5 \mathrm{mmol}, 80 \%$ yield): ${ }^{1} \mathrm{H}$ NMR $\left(400 \mathrm{MHz}, \mathrm{CDCl}_{3}\right) \delta 7.35-7.29(\mathrm{~m}, 2 \mathrm{H}), 6.90-6.84(\mathrm{~m}, 2 \mathrm{H})$, 
$5.66(\mathrm{dqd}, \mathrm{J}=10.7,6.8,1.1 \mathrm{~Hz}, 1 \mathrm{H}), 5.36-5.29(\mathrm{~m}, 1 \mathrm{H}), 3.85(\mathrm{dd}, \mathrm{J}=11.8,7.9 \mathrm{~Hz}, 1 \mathrm{H}), 3.80(\mathrm{~s}, 3 \mathrm{H}), 3.70$ (dd, J = 11.8, $4.6 \mathrm{~Hz}, 1 \mathrm{H}), 1.97(\mathrm{dd}, \mathrm{J}=14.5,8.6 \mathrm{~Hz}, 1 \mathrm{H}), 1.80(\mathrm{dd}, \mathrm{J}=6.8,1.7 \mathrm{~Hz}, 3 \mathrm{H}$ ), $1.33(\mathrm{dd}, \mathrm{J}=8.5,4.8$ $\mathrm{Hz}, 1 \mathrm{H}), 0.95-0.90$ (m, 1H). ${ }^{13} \mathrm{C}$ NMR $\left(101 \mathrm{MHz} \mathrm{CDCl}_{3}\right) \delta 158.4,135.9,130.0,129.0,126.8,113.9,68.0$, 55.3, 34.0, 22.8, 20.2, 13.4. HRMS m/z: (APCI) [M-OH] ${ }^{+}$, calculated for $\mathrm{C}_{14} \mathrm{H}_{17} \mathrm{O} ; 201.1274$; found 201.1292.

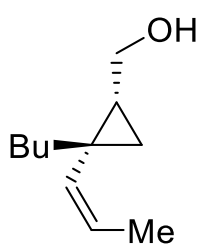

$\left(\left(1 R^{*}, 2 R^{*}\right)-2-\right.$ butyl-2-((Z)-prop-1-en-1-yl)cyclopropyl)methanol 1j (from cyclopropenyl methanol I-c, 2 mmol, 78\% yield): ${ }^{1} \mathrm{H} \mathrm{NMR}\left(400 \mathrm{MHz}, \mathrm{CDCl}_{3}\right) \delta 5.57(\mathrm{dq}, \mathrm{J}=10.8,6.8 \mathrm{~Hz}, 1 \mathrm{H}), 5.47(\mathrm{~d}, \mathrm{~J}=10.8 \mathrm{~Hz}, 1 \mathrm{H}), 3.54$ $-3.43(\mathrm{~m}, 2 \mathrm{H}), 1.69(\mathrm{~d}, \mathrm{~J}=6.8 \mathrm{~Hz}, 3 \mathrm{H}), 1.35-1.22(\mathrm{~m}, 6 \mathrm{H}), 0.97-0.92(\mathrm{~m}, 1 \mathrm{H}), 0.84(\mathrm{t}, \mathrm{J}=7.1 \mathrm{~Hz}, 3 \mathrm{H}), 0.73$ (dd, J = 8.4, $4.5 \mathrm{~Hz}, 1 \mathrm{H}), 0.42(\mathrm{t}, \mathrm{J}=4.8 \mathrm{~Hz}, 1 \mathrm{H}) .{ }^{13} \mathrm{C} \mathrm{NMR}\left(101 \mathrm{MHz}, \mathrm{CDCl}_{3}\right) \delta$ 130.0, 129.3, 64.2, 40.0, 29.4, 25.7, 24.2, 22.9, 17.9, 14.3, 14.1. HRMS m/z: (APCI) $[\mathrm{M}-\mathrm{OH}]^{+}$, calculated for $\mathrm{C}_{11} \mathrm{H}_{19}$; 151.1481; found 151.1490 .

\section{General procedure for the tandem Heck arylation - ring opening with arylboronic acids (Scheme 3)}

In a dry one-neck flask under $\mathrm{O}_{2}$ (balloon, $\left.1 \mathrm{~atm}\right)$, were added 5-(2-pyridyl)-1,3-oxazole (9 mol\%), $\left[\mathrm{Pd}(\mathrm{MeCN})_{2}(\mathrm{OTs})_{2}\right](7.5 \mathrm{~mol} \%), \mathrm{Cu}(\mathrm{OTf})_{2}(5 \mathrm{~mol} \%)$, molecular sieves $3 \AA$ ( $100 \% \mathrm{w} / \mathrm{w}$ substrate) and vinylcyclopropyl carbinol 1 (1 equiv., $0.2 \mathrm{mmol}$ for all reactions, unless otherwise noticed) in DMA (8 $\mathrm{mL} / \mathrm{mmol}$ substrate). The corresponding arylboronic acid ( 3 equiv) was then rapidly poured into the stirring reaction mixture at $55^{\circ} \mathrm{C}$ for $18 \mathrm{~h}$. After completion of the reaction, the reaction mixture was diluted with brine and EtOAc. The phases were separated and the aqueous phase was successively washed three times with EtOAc. The combined organic phases were then dried over anhydrous $\mathrm{MgSO}_{4}$ and concentrated under reduced pressure. In the relevant cases, a reduction of the aldehyde was performed: the crude mixture was dissolved in $5 \mathrm{ml}$ of methanol and $\mathrm{NaBH}_{4}$ (2equiv) was added at $0{ }^{\circ} \mathrm{C}$. The mixture was stirred for an hour at room temperature and quenched with an aqueous solution of $\mathrm{HCl} 1 \mathrm{M}(10 \mathrm{ml})$. The reaction mixture was diluted with brine and EtOAc. The phases were separated and the aqueous phase was successively extracted three times with EtOAc. The combined organic phases were then dried over anhydrous $\mathrm{MgSO}_{4}$ and concentrated under reduced pressure. Purification of the crude reaction mixture by column chromatography afforded the desired product $\mathbf{2}$.<smiles>Cc1ccc(C(C)(C)/C=C/CC(O)c2ccccc2)cc1</smiles>

$\left(2 S^{*}, 6 R^{*}, E\right)-2-p h e n y l-6-(p-t o l y l)$ hept-4-en-1-ol $2 \mathrm{e}\left(63 \%\right.$ yield): ${ }^{1} \mathrm{H}$ NMR $\left(400 \mathrm{MHz}, \mathrm{CDCl}_{3}\right) \delta 7.25$ (t, J = 7.5 $\mathrm{Hz}, 1 \mathrm{H}), 7.19-7.17(\mathrm{~m}, 1 \mathrm{H}), 7.13(\mathrm{~d}, \mathrm{~J}=7.8 \mathrm{~Hz}, 1 \mathrm{H}), 6.99(\mathrm{~d}, \mathrm{~J}=7.6 \mathrm{~Hz}, 1 \mathrm{H}), 6.90(\mathrm{~d}, \mathrm{~J}=7.5 \mathrm{~Hz}, 1 \mathrm{H}), 5.50$ $(d d, J=15.3,6.9 \mathrm{~Hz}, 1 \mathrm{H}), 5.33-5.24(\mathrm{~m}, 1 \mathrm{H}), 3.74(\mathrm{dd}, \mathrm{J}=10.8,5.6 \mathrm{~Hz}, 1 \mathrm{H}), 3.70-3.64(\mathrm{~m}, 1 \mathrm{H}), 3.25(\mathrm{p}, \mathrm{J}$ $=6.9 \mathrm{~Hz}, 1 \mathrm{H}), 2.85-2.74(\mathrm{~m}, 1 \mathrm{H}), 2.35(\mathrm{dt}, \mathrm{J}=14.4,7.3 \mathrm{~Hz}, 1 \mathrm{H}), 2.27(\mathrm{t}, \mathrm{J}=7.0 \mathrm{~Hz}, 1 \mathrm{H}), 2.24(\mathrm{~s}, 1 \mathrm{H}), 1.19$ 
(d, J = 6.5 Hz, 2H). ${ }^{13} \mathrm{C}$ NMR (101 MHz, CDCl3) $\delta 143.0,142.1,137.5,135.4,129.0,128.7,128.1,127.0$, $126.8,126.3,66.9,48.6,41.7,35.6,21.4,21.0$. HRMS m/z: (APCI) $[\mathrm{M}+\mathrm{H}]^{+}$, calculated for $\mathrm{C}_{20} \mathrm{H}_{25} \mathrm{O} ; 281.1900$; found 281.1890 .

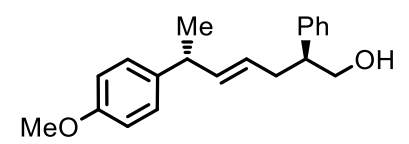

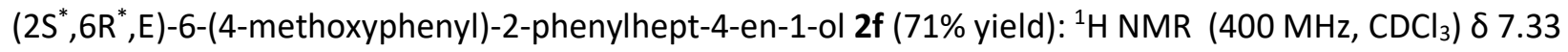
$(\mathrm{t}, \mathrm{J}=7.5 \mathrm{~Hz}, 1 \mathrm{H}), 7.26-7.22(\mathrm{~m}, 1 \mathrm{H}), 7.20(\mathrm{~d}, \mathrm{~J}=7.2 \mathrm{~Hz}, 1 \mathrm{H}), 6.99(\mathrm{~d}, \mathrm{~J}=8.6 \mathrm{~Hz}, 1 \mathrm{H}), 6.80(\mathrm{~d}, \mathrm{~J}=8.6 \mathrm{~Hz}$, $1 \mathrm{H}), 5.55(\mathrm{dd}, \mathrm{J}=15.3,6.9 \mathrm{~Hz}, 1 \mathrm{H}), 5.39-5.30(\mathrm{~m}, 1 \mathrm{H}), 3.85-3.72(\mathrm{~m}, 3 \mathrm{H}), 3.78(\mathrm{~s}, 1 \mathrm{H}), 3.31(\mathrm{p}, \mathrm{J}=7.0 \mathrm{~Hz}$, $1 \mathrm{H}), 2.91-2.83(\mathrm{~m}, 1 \mathrm{H}), 2.43(\mathrm{dt}, \mathrm{J}=14.2,7.2 \mathrm{~Hz}, 1 \mathrm{H}), 2.33(\mathrm{dt}, \mathrm{J}=13.9,7.1 \mathrm{~Hz}, 1 \mathrm{H}), 1.25(\mathrm{~d}, \mathrm{~J}=6.9 \mathrm{~Hz}$, 2H). ${ }^{13} \mathrm{C}$ NMR $\left(101 \mathrm{MHz}, \mathrm{CDCl}_{3}\right) \delta 157.8,142.1,138.1,137.6,128.7,128.1,128.0,126.8,126.2,113.7,66.9$, 55.3, 48.6, 41.2, 35.5, 21.4. HRMS m/z: (APCI) $[\mathrm{M}+\mathrm{H}]^{+}$, calculated for $\mathrm{C}_{20} \mathrm{H}_{25} \mathrm{O}_{2} ; 297.1849$; found 297.1842 .<smiles>C[C@H](/C=C/CC(O)c1ccccc1)c1ccc(Cl)cc1</smiles>

$\left(2 \mathrm{~S}^{*}, 6 \mathrm{R}^{*}, \mathrm{E}\right)-6$-(4-chlorophenyl)-2-phenylhept-4-en-1-ol $2 \mathrm{~g}$ (65\% yield): ${ }^{1} \mathrm{H} \mathrm{NMR}\left(400 \mathrm{MHz}, \mathrm{CDCl}_{3}\right) \delta 7.32$ $(\mathrm{t}, \mathrm{J}=7.3 \mathrm{~Hz}, 1 \mathrm{H}), 7.27-7.25(\mathrm{~m}, 1 \mathrm{H}), 7.21-7.18(\mathrm{~m}, 3 \mathrm{H}), 6.96(\mathrm{~d}, \mathrm{~J}=8.4 \mathrm{~Hz}, 1 \mathrm{H}), 5.50$ (dd, J = 15.3, 6.9 $\mathrm{Hz}, 1 \mathrm{H}), 5.38-5.30(\mathrm{~m}, 1 \mathrm{H}), 3.78(\mathrm{qd}, \mathrm{J}=10.9,6.7 \mathrm{~Hz}, 2 \mathrm{H}), 3.32(\mathrm{p}, \mathrm{J}=6.8 \mathrm{~Hz}, 1 \mathrm{H}), 2.92-2.83(\mathrm{~m}, 1 \mathrm{H})$, $2.45(\mathrm{dt}, \mathrm{J}=13.9,6.9 \mathrm{~Hz}, 1 \mathrm{H}), 2.38-2.28(\mathrm{~m}, 1 \mathrm{H}), 1.25(\mathrm{~d}, \mathrm{~J}=7.0 \mathrm{~Hz}, 3 \mathrm{H}) .{ }^{13} \mathrm{C} \mathrm{NMR}\left(101 \mathrm{MHz}, \mathrm{CDCl}_{3}\right) \delta$ $144.4,141.9,136.8,131.6,128.7,128.5,128.4,128.1,127.0,126.8,66.9,48.6,41.5,35.4,21.2$. HRMS $\mathrm{m} / \mathrm{z}$ : (APCI) $[\mathrm{M}+\mathrm{H}]^{+}$, calculated for $\mathrm{C}_{19} \mathrm{H}_{22} \mathrm{ClO} ; 301.1354$; found 301.1331 .

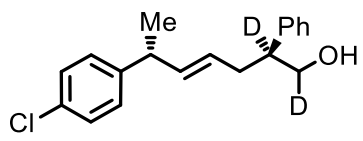

$\left(2 \mathrm{~S}^{*}, 6 \mathrm{R}^{*}, \mathrm{E}\right)-6-(4-c h l o r o p h e n y l)-2-$ phenylhept-4-en-1-ol $2 \mathrm{~g}_{\mathrm{d} 2}$ (65\% yield): ${ }^{1} \mathrm{H}$ NMR (400 MHz, $\left.\mathrm{CDCl}_{3}\right) \delta 7.32$ $(\mathrm{d}, \mathrm{J}=7.6 \mathrm{~Hz}, 3 \mathrm{H}), 7.26(\mathrm{t}, \mathrm{J}=3.6 \mathrm{~Hz}, 2 \mathrm{H}), 7.21-7.18(\mathrm{~m}, 4 \mathrm{H}), 6.96(\mathrm{~d}, \mathrm{~J}=8.4 \mathrm{~Hz}, 2 \mathrm{H}), 5.50$ (dd, J = 15.3, 6.9 $\mathrm{Hz}, 1 \mathrm{H}), 5.34(\mathrm{dt}, \mathrm{J}=14.2,7.0 \mathrm{~Hz}, 1 \mathrm{H}), 3.82-3.69(\mathrm{~m}, 1 \mathrm{H}), 3.32(\mathrm{t}, \mathrm{J}=7.0 \mathrm{~Hz}, 1 \mathrm{H}), 2.44(\mathrm{dd}, \mathrm{J}=14.0,7.2 \mathrm{~Hz}$, $1 \mathrm{H}), 2.38-2.26(\mathrm{~m}, 1 \mathrm{H}), 1.26-1.23(\mathrm{~m}, 3 \mathrm{H}) .{ }^{13} \mathrm{C} \mathrm{NMR}\left(101 \mathrm{MHz}, \mathrm{CDCl}_{3}\right) \delta$ 144.4, 141.8, 136.8, 131.6, 128.7, $128.5,128.4,128.1,127.0,126.8,41.5,35.3,29.7,21.2$. HRMS $\mathrm{m} / \mathrm{z}:(\mathrm{APCl})[\mathrm{M}+\mathrm{H}]^{+}$, calculated for $\mathrm{C}_{19} \mathrm{H}_{19} \mathrm{ClD}_{2} \mathrm{NaO} ; 325.1299$; found 325.1325 .<smiles>C[C@H](/C=C/CC(O)c1ccccc1)c1ccc2ccccc2c1</smiles>

(2S*,6R*,E)-6-(naphthalen-2-yl)-2-phenylhept-4-en-1-ol $2 \mathrm{~h}$ (56\% yield): ${ }^{1} \mathrm{H}$ NMR (400 MHz, CDCl3) $\delta 7.81$ $-7.69(\mathrm{~m}, 3 \mathrm{H}), 7.53(\mathrm{~s}, 1 \mathrm{H}), 7.47-7.39(\mathrm{~m}, 2 \mathrm{H}), 7.32(\mathrm{t}, \mathrm{J}=7.3 \mathrm{~Hz}, 2 \mathrm{H}), 7.22$ (ddd, J = 6.9, 4.9, 3.0 Hz, 3H), $5.66(\mathrm{dd}, \mathrm{J}=15.3,6.8 \mathrm{~Hz}, 1 \mathrm{H}), 5.49-5.35(\mathrm{~m}, 1 \mathrm{H}), 3.79(\mathrm{ddd}, \mathrm{J}=18.5,10.8,6.7 \mathrm{~Hz}, 2 \mathrm{H}), 3.60-3.46(\mathrm{~m}$, $1 \mathrm{H}), 2.95-2.83(\mathrm{~m}, 1 \mathrm{H}), 2.46(\mathrm{dt}, \mathrm{J}=14.2,7.2 \mathrm{~Hz}, 1 \mathrm{H}), 2.36(\mathrm{dt}, \mathrm{J}=14.2,7.2 \mathrm{~Hz}, 1 \mathrm{H}), 1.37(\mathrm{~d}, \mathrm{~J}=7.0 \mathrm{~Hz}$, $3 \mathrm{H}) .{ }^{13} \mathrm{C}$ NMR $(101 \mathrm{MHz}, \mathrm{CDCl} 3) \delta 143.5,142.0,137.2,133.6,132.1,128.7,128.1,127.9,127.7,127.6$, 
126.9, 126.8, 126.3, 125.9, 125.26, 124.9, 66.9, 48.7, 42.3, 35.5, 21.3. HRMS m/z: (APCI) [M+H]+, calculated for $\mathrm{C} 23 \mathrm{H} 25 \mathrm{O}$; 317.1900; found 317.1920.

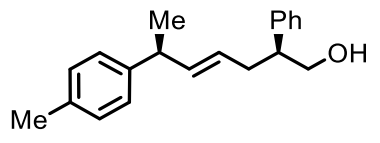

$\left(2 \mathrm{~S}^{*}, 6 \mathrm{~S}^{*}, \mathrm{E}\right)-2-$ phenyl-6-(p-tolyl)hept-4-en-1-ol 2a (62\% yield): ${ }^{1} \mathrm{H} \mathrm{NMR}\left(400 \mathrm{MHz}, \mathrm{CDCl}_{3}\right) \delta 7.35$ (t, J = 3.0 $\mathrm{Hz}, 1 \mathrm{H}), 7.34-7.31(\mathrm{~m}, 2 \mathrm{H}), 7.21(\mathrm{dd}, \mathrm{J}=5.2,3.2 \mathrm{~Hz}, 2 \mathrm{H}), 7.06(\mathrm{~d}, \mathrm{~J}=7.8 \mathrm{~Hz}, 2 \mathrm{H}), 6.94(\mathrm{~d}, \mathrm{~J}=8.0 \mathrm{~Hz}, 2 \mathrm{H})$, 5.56 (ddt, J = 15.3, 6.6, $1.1 \mathrm{~Hz}, 1 \mathrm{H}$ ), 5.36 (dtd, J = 8.2, 7.0, 1.2 Hz, 1H), 3.77 (ddd, J = 18.5, 10.9, 6.8 Hz, 2H), $3.37-3.28(\mathrm{~m}, 1 \mathrm{H}), 2.93-2.83(\mathrm{~m}, 1 \mathrm{H}), 2.44(\mathrm{dt}, \mathrm{J}=13.9,7.0 \mathrm{~Hz}, 1 \mathrm{H}), 2.34(\mathrm{dd}, \mathrm{J}=8.4,5.6 \mathrm{~Hz}, 2 \mathrm{H}), 2.31$ (s, 3H), 1.22 (d, J = $7.0 \mathrm{~Hz}, 3 \mathrm{H}) .{ }^{13} \mathrm{C}$ NMR $\left(101 \mathrm{MHz}, \mathrm{CDCl}_{3}\right) \delta 143.1,142.0,137.4,135.43,129.0,128.7$, 128.2, 127.0, 126.8, 126.2, 67.0, 48.7, 41.7, 35.6, 29.8, 21.4, 21.0. HRMS m/z: (APCI) $[\mathrm{M}+\mathrm{H}]^{+}$, calculated for $\mathrm{C}_{20} \mathrm{H}_{25} \mathrm{O} ; 281.1900$; found 281.1876 .<smiles>COc1ccc(C(C)(C)/C=C/CC(O)c2ccccc2)cc1</smiles>

$\left(2 \mathrm{~S}^{*}, 6 \mathrm{~S}^{*}, \mathrm{E}\right)-6$-(4-methoxyphenyl)-2-phenylhept-4-en-1-ol $\mathbf{2 b}$ (69\% yield): ${ }^{1} \mathrm{H} \mathrm{NMR}\left(400 \mathrm{MHz}, \mathrm{CDCl}_{3}\right) \delta 7.36$ $-7.31(\mathrm{~m}, 1 \mathrm{H}), 7.28-7.24(\mathrm{~m}, 1 \mathrm{H}), 7.21(\mathrm{dd}, \mathrm{J}=5.2,3.2 \mathrm{~Hz}, 1 \mathrm{H}), 6.98-6.94(\mathrm{~m}, 1 \mathrm{H}), 6.81-6.76(\mathrm{~m}, 1 \mathrm{H})$, 5.55 (ddt, J = 15.3, 6.5, $1.1 \mathrm{~Hz}, 1 \mathrm{H}$ ), 5.35 (dtd, J = 8.3, 7.0, $1.2 \mathrm{~Hz}, 1 \mathrm{H}$ ), $3.81(\mathrm{dd}, \mathrm{J}=7.2,3.3 \mathrm{~Hz}, 1 \mathrm{H}), 3.78(\mathrm{~s}$, $1 \mathrm{H}), 3.75(\mathrm{dd}, \mathrm{J}=10.8,7.7 \mathrm{~Hz}, 1 \mathrm{H}), 3.31(\mathrm{p}, \mathrm{J}=7.0 \mathrm{~Hz}, 1 \mathrm{H}), 2.92-2.83(\mathrm{~m}, 1 \mathrm{H}), 2.45(\mathrm{dt}, \mathrm{J}=13.9,6.9 \mathrm{~Hz}$, $1 \mathrm{H}), 2.32(\mathrm{dt}, \mathrm{J}=20.9,7.3 \mathrm{~Hz}, 1 \mathrm{H}), 1.21(\mathrm{~d}, \mathrm{~J}=7.0 \mathrm{~Hz}, 1 \mathrm{H}) .{ }^{13} \mathrm{C} \mathrm{NMR}\left(101 \mathrm{MHz}, \mathrm{CDCl}_{3}\right) \delta 157.8,142.0,138.3$, $137.5,128.7,128.2,128.0,126.8,126.1,113.7,67.0,55.3,48.7,41.2,35.6,21.4 . H R M S m / z:(A P C l) ~[M+H]^{+}$, calculated for $\mathrm{C}_{20} \mathrm{H}_{25} \mathrm{O}_{2} ; 297.1849$; found 297.1838.<smiles>CC(/C=C/CC(O)c1ccccc1)c1ccc(Cl)cc1</smiles>

(2S*,6S*,E)-6-(4-chlorophenyl)-2-phenylhept-4-en-1-ol 2c (61\% yield): ${ }^{1} \mathrm{H}$ NMR (400 MHz, $\left.\mathrm{CDCl}_{3}\right) \delta 7.36$ $7.31(\mathrm{~m}, 3 \mathrm{H}), 7.28(\mathrm{t}, \mathrm{J}=1.4 \mathrm{~Hz}, 1 \mathrm{H}), 7.21-7.17(\mathrm{~m}, 4 \mathrm{H}), 6.95-6.91(\mathrm{~m}, 2 \mathrm{H}), 5.51(\mathrm{ddt}, \mathrm{J}=15.3,6.3,1.1$ $\mathrm{Hz}, 1 \mathrm{H}), 5.35$ (dtd, J = 8.2, 7.0, $1.2 \mathrm{~Hz}, 1 \mathrm{H}), 3.77(\mathrm{qd}, \mathrm{J}=10.9,6.8 \mathrm{~Hz}, 2 \mathrm{H}), 3.32(\mathrm{p}, \mathrm{J}=7.0 \mathrm{~Hz}, 1 \mathrm{H}), 2.93-$ $2.83(\mathrm{~m}, 1 \mathrm{H}), 2.46(\mathrm{dt}, \mathrm{J}=13.4,6.7 \mathrm{~Hz}, 1 \mathrm{H}), 2.32(\mathrm{dt}, \mathrm{J}=14.4,6.8 \mathrm{~Hz}, 1 \mathrm{H}), 1.20(\mathrm{~d}, \mathrm{~J}=7.0 \mathrm{~Hz}, 2 \mathrm{H}) .{ }^{13} \mathrm{C} \mathrm{NMR}$ $\left(101 \mathrm{MHz} \mathrm{CDCl}_{3}\right) \delta 144.6,141.9,136.6,131.6,128.7,128.5,128.4,128.2,126.83,67.0,48.7,41.4,35.5$, 21.2. HRMS m/z: (APCl) $[\mathrm{M}+\mathrm{H}]^{+}$, calculated for $\mathrm{C}_{19} \mathrm{H}_{22} \mathrm{OCl}$; 301.1354; found 301.1344.<smiles>C[C@H](/C=C/CC(O)c1ccccc1)c1ccc2ccccc2c1</smiles>

(2S*,6S*,E)-6-(naphthalen-2-yl)-2-phenylhept-4-en-1-ol 2d (53\% yield): ${ }^{1} \mathrm{H}$ NMR (400 MHz, CDCl3) $\delta 7.76$ (ddd, J = 18.7, 10.0, 7.9 Hz, 3H), 7.51 (s, 1H), 7.46- $7.39(\mathrm{~m}, 2 \mathrm{H}), 7.34$ (dd, J = 10.0, $4.5 \mathrm{~Hz}, 2 \mathrm{H}), 7.29-7.24$ $(\mathrm{m}, 2 \mathrm{H}), 7.23-7.17(\mathrm{~m}, 3 \mathrm{H}), 5.65(\mathrm{dd}, \mathrm{J}=15.3,6.6 \mathrm{~Hz}, 1 \mathrm{H}), 5.48-5.37(\mathrm{~m}, 1 \mathrm{H}), 3.78(\mathrm{ddd}, \mathrm{J}=18.5,10.9$, $6.7 \mathrm{~Hz}, 2 \mathrm{H}), 3.53(\mathrm{p}, \mathrm{J}=6.9 \mathrm{~Hz}, 1 \mathrm{H}), 2.96-2.84(\mathrm{~m}, 1 \mathrm{H}), 2.47(\mathrm{dt}, \mathrm{J}=13.9,6.9 \mathrm{~Hz}, 1 \mathrm{H}), 2.41-2.31(\mathrm{~m}, 1 \mathrm{H})$, $1.33(\mathrm{~d}, \mathrm{~J}=7.0 \mathrm{~Hz}, 3 \mathrm{H}) .{ }^{13} \mathrm{C} \mathrm{NMR}\left(101 \mathrm{MHz}, \mathrm{CDCl}_{3}\right) \delta 143.68,142.10,137.16,133.72,132.25,128.79$, 
$128.28,127.99,127.76,127.69,126.93,126.86,126.38,125.96,125.35,125.05,67.10,48.77,42.35$, 35.67, 21.33. HRMS m/z: (APCI) [M+H]+, calculated for $\mathrm{C} 23 \mathrm{H} 250$; 317.1900; found 317.1908.<smiles>CC(=O)c1ccc(C(C)(C)CCC(CO)c2ccccc2)cc1</smiles>

Methyl 4-((2R* $\left.\left.6 \mathrm{~S}^{*}, \mathrm{E}\right)-7-h y d r o x y-6-p h e n y l h e p t-3-e n-2-y l\right)$ benzoate $2 \mathrm{i}$ (38\% yield): ${ }^{1} \mathrm{H}$ NMR (400 MHz, $\mathrm{CDCl} 3) \delta 7.95-7.87(\mathrm{~m}, 2 \mathrm{H}), 7.35-7.29(\mathrm{~m}, 2 \mathrm{H}), 7.26-7.22(\mathrm{~m}, 1 \mathrm{H}), 7.22-7.16(\mathrm{~m}, 2 \mathrm{H}), 7.11(\mathrm{~d}, \mathrm{~J}=8.2$ $\mathrm{Hz}, 2 \mathrm{H}), 5.53(\mathrm{dd}, \mathrm{J}=15.3,6.9 \mathrm{~Hz}, 1 \mathrm{H}), 5.36(\mathrm{dt}, \mathrm{J}=7.1,3.9 \mathrm{~Hz}, 1 \mathrm{H}), 3.90(\mathrm{~s}, 3 \mathrm{H}), 3.77(\mathrm{tt}, \mathrm{J}=15.7,7.8 \mathrm{~Hz}$, $2 \mathrm{H}), 3.40(\mathrm{p}, \mathrm{J}=6.9 \mathrm{~Hz}, 1 \mathrm{H}), 2.94-2.80(\mathrm{~m}, 1 \mathrm{H}), 2.45(\mathrm{dt}, \mathrm{J}=13.8,6.9 \mathrm{~Hz}, 1 \mathrm{H}), 2.39-2.28(\mathrm{~m}, 1 \mathrm{H}), 1.28(\mathrm{~d}$, $\mathrm{J}=7.0 \mathrm{~Hz}, 3 \mathrm{H}) .{ }^{13} \mathrm{C}$ NMR $\left(101 \mathrm{MHz}, \mathrm{CDCl}_{3}\right) \delta 167.19,151.47,141.85,136.36,129.72,128.70,128.13$, $127.90,127.38,127.16,126.86,66.91,52.02,48.60,42.19,35.40,21.13$.<smiles>COc1ccc([C@](C)(O)/C=C/CC(O)c2ccccc2)cc1OC</smiles>

$\left(2 \mathrm{~S}^{*}, 6 \mathrm{R}^{*}, \mathrm{E}\right)-6-(3,4-d i m e t h o x y p h e n y l)-2-p h e n y l h e p t-4-e n-1-o l ~ 2 \mathrm{j}\left(40 \%\right.$ yield): ${ }^{1} \mathrm{H} \mathrm{NMR}\left(400 \mathrm{MHz}, \mathrm{CDCl}_{3}\right) \delta$ $7.32(\mathrm{t}, \mathrm{J}=7.3 \mathrm{~Hz}, 1 \mathrm{H}), 7.20(\mathrm{~d}, \mathrm{~J}=7.6 \mathrm{~Hz}, 1 \mathrm{H}), 6.77(\mathrm{~d}, \mathrm{~J}=8.2 \mathrm{~Hz}, 1 \mathrm{H}), 6.64(\mathrm{~d}, \mathrm{~J}=7.3 \mathrm{~Hz}, 1 \mathrm{H}), 5.59(\mathrm{dd}, \mathrm{J}=$ 15.4, $6.7 \mathrm{~Hz}, 1 \mathrm{H}), 5.41-5.32(\mathrm{~m}, 1 \mathrm{H}), 3.85(\mathrm{~s}, 1 \mathrm{H}), 3.84(\mathrm{~s}, 1 \mathrm{H}), 3.78-3.72(\mathrm{~m}, 1 \mathrm{H}), 3.35-3.28(\mathrm{~m}, 1 \mathrm{H})$, $2.93-2.81(\mathrm{~m}, 1 \mathrm{H}), 2.40$ (ddt, J = 34.4, 13.9, 7.0 Hz, 1H), 1.27 (d, J = 7.1 Hz, 3H). ${ }^{13} \mathrm{C} \mathrm{NMR}\left(101 \mathrm{MHz}, \mathrm{CDCl}_{3}\right)$ $\delta 142.2,138.8,137.5,128.7,128.1,126.8,126.4,122.9,118.8,111.2,110.6,66.9,56.0,48.6,41.8,35.5$, 21.5. HRMS m/z: (APCl) $[\mathrm{M}+\mathrm{H}]^{+}$, calculated for $\mathrm{C}_{21} \mathrm{H}_{27} \mathrm{O}_{3} ; 327.1955$; found 327.1964 .<smiles>C[C@H](/C=C/CC(O)c1ccc(C(F)(F)F)cc1)c1ccccc1</smiles>

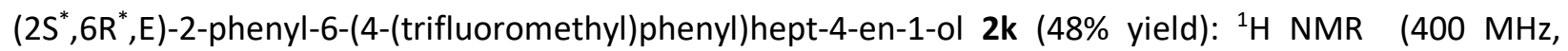
$\left.\mathrm{CDCl}_{3}\right) \delta 7.41(\mathrm{~d}, \mathrm{~J}=8.1 \mathrm{~Hz}, 2 \mathrm{H}), 7.24(\mathrm{t}, \mathrm{J}=7.3 \mathrm{~Hz}, 2 \mathrm{H}), 7.18-7.14(\mathrm{~m}, 1 \mathrm{H}), 7.13-7.09(\mathrm{~m}, 2 \mathrm{H}), 7.07(\mathrm{~d}, \mathrm{~J}$ $=8.2 \mathrm{~Hz}, 2 \mathrm{H}), 5.44(\mathrm{dd}, \mathrm{J}=15.3,6.9 \mathrm{~Hz}, 1 \mathrm{H}), 5.29(\mathrm{dtd}, \mathrm{J}=7.9,7.0,0.9 \mathrm{~Hz}, 1 \mathrm{H}), 3.71(\mathrm{qd}, \mathrm{J}=10.9,6.7 \mathrm{~Hz}$, $2 \mathrm{H}), 3.33(\mathrm{p}, \mathrm{J}=7.0 \mathrm{~Hz}, 1 \mathrm{H}), 2.84-2.76(\mathrm{~m}, 1 \mathrm{H}), 2.39(\mathrm{dt}, \mathrm{J}=13.7,6.8 \mathrm{~Hz}, 1 \mathrm{H}), 2.30-2.22(\mathrm{~m}, 1 \mathrm{H}), 1.21(\mathrm{~d}$, $\mathrm{J}=7.0 \mathrm{~Hz}, 3 \mathrm{H}) .{ }^{13} \mathrm{C} \mathrm{NMR}\left(101 \mathrm{MHz}, \mathrm{CDCl}_{3}\right) \delta 150.1,141.8,136.3,128.7,128.1,127.5,127.4,126.87,125.2$, 125.2, 67.0, 48.6, 42.0, 35.4, 21.1. HRMS m/z: (APCI) $[\mathrm{M}+\mathrm{H}]^{+}$, calculated for $\mathrm{C}_{20} \mathrm{H}_{22} \mathrm{~F}_{3} \mathrm{O} ; 335.1617$; found 335.1636 .<smiles>OCC(O)c1ccccc1</smiles>

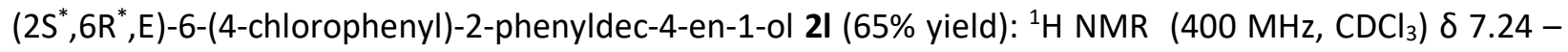
$7.21(\mathrm{~m}, 2 \mathrm{H}), 7.17-7.08(\mathrm{~m}, 5 \mathrm{H}), 6.91-6.87(\mathrm{~m}, 2 \mathrm{H}), 5.39(\mathrm{dd}, \mathrm{J}=15.3,7.8 \mathrm{~Hz}, 1 \mathrm{H}), 5.28-5.19(\mathrm{~m}, 1 \mathrm{H})$, $3.69(\mathrm{qd}, \mathrm{J}=10.9,6.7 \mathrm{~Hz}, 2 \mathrm{H}), 3.02(\mathrm{q}, \mathrm{J}=7.5 \mathrm{~Hz}, 1 \mathrm{H}), 2.81-2.73(\mathrm{~m}, 1 \mathrm{H}), 2.35(\mathrm{dt}, \mathrm{J}=14.0,7.0 \mathrm{~Hz}, 1 \mathrm{H})$, $2.23(\mathrm{dt}, \mathrm{J}=13.7,6.9 \mathrm{~Hz}, 1 \mathrm{H}), 1.49(\mathrm{dd}, \mathrm{J}=15.2,7.6 \mathrm{~Hz}, 3 \mathrm{H}), 1.22-1.16(\mathrm{~m}, 7 \mathrm{H}), 0.77(\mathrm{t}, \mathrm{J}=7.2 \mathrm{~Hz}, 4 \mathrm{H}) .{ }^{13} \mathrm{C}$ NMR $\left(101 \mathrm{MHz}, \mathrm{CDCl}_{3}\right) \delta 143.7,141.9,135.9,131.5,128.8,128.7,128.4,128.1,127.8,126.8,66.9,48.6$, 
48.0, 35.5, 35.5, 29.8, 29.7, 22.6, 14.0. HRMS m/z: (APCl) $[\mathrm{M}+\mathrm{H}]^{+}$, calculated for $\mathrm{C}_{22} \mathrm{H}_{28} \mathrm{ClO}$; 343.1823; found 343.1841 .<smiles>OCC(O)c1ccccc1</smiles>

$\left(2 \mathrm{~S}^{*}, 6 \mathrm{~S}^{*}, \mathrm{E}\right)-6$-(4-chlorophenyl)-2-phenyldec-4-en-1-ol $2 \mathrm{~m}$ (61\% yield): ${ }^{1} \mathrm{H} \mathrm{NMR}\left(400 \mathrm{MHz}, \mathrm{CDCl}_{3}\right) \delta 7.25-$ $7.22(\mathrm{~m}, 1 \mathrm{H}), 7.17-7.09(\mathrm{~m}, 2 \mathrm{H}), 6.91-6.87(\mathrm{~m}, 1 \mathrm{H}), 5.37(\mathrm{dd}, \mathrm{J}=15.3,7.6 \mathrm{~Hz}, 1 \mathrm{H}), 5.29-5.20(\mathrm{~m}, 1 \mathrm{H})$, $3.72-3.63(\mathrm{~m}, 1 \mathrm{H}), 3.00(\mathrm{q}, \mathrm{J}=7.4 \mathrm{~Hz}, 1 \mathrm{H}), 2.77(\mathrm{dd}, \mathrm{J}=14.7,7.0 \mathrm{~Hz}, 1 \mathrm{H}), 2.36(\mathrm{dt}, \mathrm{J}=13.3,6.6 \mathrm{~Hz}, 1 \mathrm{H})$, $2.28-2.20(\mathrm{~m}, 1 \mathrm{H}), 1.44(\mathrm{ddd}, \mathrm{J}=13.6,9.1,2.3 \mathrm{~Hz}, 1 \mathrm{H}), 1.18(\mathrm{~s}, 1 \mathrm{H}), 0.74(\mathrm{t}, \mathrm{J}=7.3 \mathrm{~Hz}, 2 \mathrm{H}) .{ }^{13} \mathrm{C}$ NMR $(101$ $\left.\mathrm{MHz}_{2} \mathrm{CDCl}_{3}\right) \delta 143.8,141.8,135.8,131.5,128.8,128.7,128.4,128.2,127.6,126.8,67.0,48.6,48.0,35.5$, 35.4, 29.75, 29.6, 22.6, 14.0. HRMS m/z: (APCl) $[\mathrm{M}+\mathrm{H}]^{+}$, calculated for $\mathrm{C}_{22} \mathrm{H}_{28} \mathrm{ClO}$; 343.1823; found 343.1839.<smiles>COc1ccc(C(Br)/C=C/CC(O)c2ccccc2)cc1</smiles>

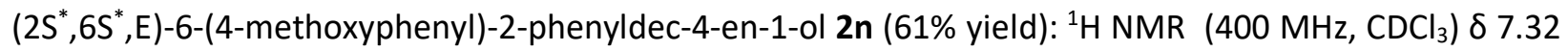
$(\mathrm{t}, \mathrm{J}=7.3 \mathrm{~Hz}, 2 \mathrm{H}), 7.23(\mathrm{~d}, \mathrm{~J}=7.4 \mathrm{~Hz}, 1 \mathrm{H}), 7.21-7.17(\mathrm{~m}, 2 \mathrm{H}), 7.01-6.96(\mathrm{~m}, 2 \mathrm{H}), 6.82-6.78(\mathrm{~m}, 2 \mathrm{H}), 5.48$ $(\mathrm{dd}, \mathrm{J}=15.2,7.7 \mathrm{~Hz}, 1 \mathrm{H}), 5.31(\mathrm{dt}, \mathrm{J}=10.7,6.9 \mathrm{~Hz}, 1 \mathrm{H}), 3.81(\mathrm{dd}, \mathrm{J}=5.8,3.2 \mathrm{~Hz}, 1 \mathrm{H}), 3.78(\mathrm{~s}, 2 \mathrm{H}), 3.77-$ $3.69(\mathrm{~m}, 1 \mathrm{H}), 3.05(\mathrm{dt}, \mathrm{J}=12.6,6.3 \mathrm{~Hz}, 1 \mathrm{H}), 2.90-2.82(\mathrm{~m}, 1 \mathrm{H}), 2.42(\mathrm{dt}, \mathrm{J}=13.5,6.7 \mathrm{~Hz}, 1 \mathrm{H}), 2.36-2.27$ $(\mathrm{m}, 1 \mathrm{H}), 1.52(\mathrm{dd}, \mathrm{J}=15.4,7.7 \mathrm{~Hz}, 2 \mathrm{H}), 1.26(\mathrm{~s}, 4 \mathrm{H}), 0.82(\mathrm{t}, \mathrm{J}=7.3 \mathrm{~Hz}, 3 \mathrm{H}) .{ }^{13} \mathrm{C} \mathrm{NMR}\left(101 \mathrm{MHz}, \mathrm{CDCl}_{3}\right) \delta$ 157.8, 142.0, 137.5, 136.7, 128.6, 128.3, 128.2, 126.8 126.8, 113.7, 67.1, 55.3, 48.7, 47.8, 35.6, 29.8, 29.6, 22.6, 14.0. HRMS m/z: (APCI) [M-H] $]^{+}$, calculated for $\mathrm{C}_{23} \mathrm{H}_{29} \mathrm{O}_{2} ; 337.2162$; found 337.2157.<smiles>COc1ccc(C(C=CC=CC(C)(C#N)c2ccc(OC)cc2)CO)cc1</smiles>

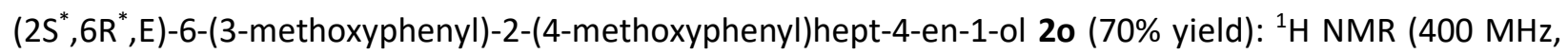
$\left.\mathrm{CDCl}_{3}\right) \delta 7.18(\mathrm{t}, \mathrm{J}=8.0 \mathrm{~Hz}, 1 \mathrm{H}), 7.14-7.10(\mathrm{~m}, 2 \mathrm{H}), 6.88-6.83(\mathrm{~m}, 2 \mathrm{H}), 6.74-6.67(\mathrm{~m}, 3 \mathrm{H}), 5.58(\mathrm{dd}, \mathrm{J}=$ $15.3,6.9 \mathrm{~Hz}, 1 \mathrm{H}), 5.42-5.32(\mathrm{~m}, 1 \mathrm{H}), 3.80(\mathrm{~s}, 3 \mathrm{H}), 3.78(\mathrm{~s}, 3 \mathrm{H}), 3.70(\mathrm{dd}, \mathrm{J}=10.8,7.8 \mathrm{~Hz}, 1 \mathrm{H}), 3.34(\mathrm{p}, \mathrm{J}=$ $6.9 \mathrm{~Hz}, 1 \mathrm{H}), 2.87-2.77(\mathrm{~m}, 1 \mathrm{H}), 2.40(\mathrm{dt}, \mathrm{J}=14.3,7.2 \mathrm{~Hz}, 1 \mathrm{H}), 2.29(\mathrm{dt}, \mathrm{J}=14.1,7.1 \mathrm{~Hz}, 1 \mathrm{H}), 1.28(\mathrm{~d}, \mathrm{~J}=7.0$ $\mathrm{Hz}, 3 \mathrm{H}) .{ }^{13} \mathrm{C}$ NMR $\left(101 \mathrm{MHz}, \mathrm{CDCl}_{3}\right) \delta 159.6,158.4,147.8,137.0,133.9,129.3,129.0,126.7,119.6,114.1$, 113.1, 111.0, 67.0, 55.3, 55.2, 47.8, 42.2, 35.7, 29.7, 21.3. HRMS m/z: (APCI) $[\mathrm{M}-\mathrm{OH}]^{+}$, calculated for $\mathrm{C}_{21} \mathrm{H}_{25} \mathrm{O}_{2} ; 309.1849$; found 309.1863 . 


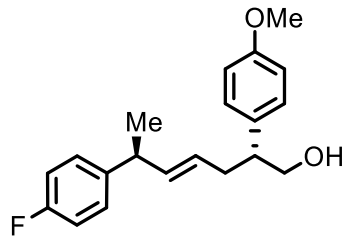

$\left(2 \mathrm{~S}^{*}, 6 \mathrm{R}^{*}, \mathrm{E}\right)-6$-(4-fluorophenyl)-2-(4-methoxyphenyl)hept-4-en-1-ol $2 \mathrm{p}$ (51\% yield): ${ }^{1} \mathrm{H}$ NMR (400 MHz, $\left.\mathrm{CDCl}_{3}\right) \delta 7.13-7.08(\mathrm{~m}, 2 \mathrm{H}), 7.02-6.97(\mathrm{~m}, 2 \mathrm{H}), 6.94-6.89(\mathrm{~m}, 2 \mathrm{H}), 6.87-6.85(\mathrm{~m}, 2 \mathrm{H}), 5.50(\mathrm{dd}, \mathrm{J}=$ $15.3,6.9 \mathrm{~Hz}, 1 \mathrm{H}), 5.38-5.28(\mathrm{~m}, 1 \mathrm{H}), 3.80(\mathrm{~s}, 3 \mathrm{H}), 3.76(\mathrm{~d}, \mathrm{~J}=5.7 \mathrm{~Hz}, 1 \mathrm{H}), 3.70(\mathrm{dd}, \mathrm{J}=10.8,7.8 \mathrm{~Hz}, 1 \mathrm{H})$, $3.33(\mathrm{p}, \mathrm{J}=7.1 \mathrm{~Hz}, 1 \mathrm{H}), 2.86-2.76(\mathrm{~m}, 1 \mathrm{H}), 2.41(\mathrm{dt}, \mathrm{J}=13.8,6.9 \mathrm{~Hz}, 1 \mathrm{H}), 2.33-2.23(\mathrm{~m}, 1 \mathrm{H}), 1.26(\mathrm{~d}, \mathrm{~J}=$ $2.6 \mathrm{~Hz}, 3 \mathrm{H}) .{ }^{13} \mathrm{C}$ NMR $\left(101 \mathrm{MHz}, \mathrm{CDCl}_{3}\right) \delta 162.4,158.5,141.6,137.0,133.8,129.1,128.5,128.4,126.8$, 115.0, 114.8, 114.1, 67.0, 55.3, 47.8, 41.4, 35.6, 29.7, 21.4. HRMS m/z: (APCI) [M-OH] ${ }^{+}$, calculated for $\mathrm{C}_{20} \mathrm{H}_{22} \mathrm{FO} ; 297.1649$; found 297.1618.

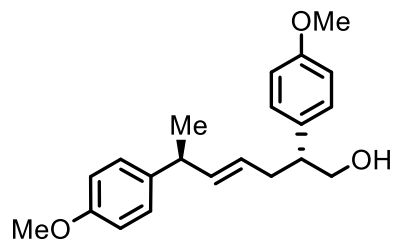

$\left(2 \mathrm{~S}^{*}, 6 \mathrm{R}^{*}, \mathrm{E}\right)-2,6$-bis(4-methoxyphenyl)hept-4-en-1-ol 2q (72\% yield): ${ }^{1} \mathrm{H} \mathrm{NMR}$ (400 MHz, $\left.\mathrm{CDCl}_{3}\right) \delta 7.13-$ $7.09(\mathrm{~m}, 2 \mathrm{H}), 7.00-6.95(\mathrm{~m}, 2 \mathrm{H}), 6.89-6.85(\mathrm{~m}, 2 \mathrm{H}), 6.82-6.78(\mathrm{~m}, 2 \mathrm{H}), 5.53(\mathrm{dd}, \mathrm{J}=15.3,6.9 \mathrm{~Hz}, 1 \mathrm{H})$, $5.38-5.29(\mathrm{~m}, 1 \mathrm{H}), 3.80(\mathrm{~s}, 3 \mathrm{H}), 3.79(\mathrm{~s}, 1 \mathrm{H}), 3.78(\mathrm{~s}, 3 \mathrm{H}), 3.73-3.66(\mathrm{~m}, 1 \mathrm{H}), 3.35-3.27(\mathrm{~m}, 1 \mathrm{H}), 2.86-$ $2.77(\mathrm{~m}, 1 \mathrm{H}), 2.40(\mathrm{dt}, \mathrm{J}=14.2,7.2 \mathrm{~Hz}, 1 \mathrm{H}), 2.33-2.24(\mathrm{~m}, 1 \mathrm{H}), 1.26(\mathrm{~d}, \mathrm{~J}=3.0 \mathrm{~Hz}, 3 \mathrm{H}) .{ }^{13} \mathrm{C} \mathrm{NMR}(101 \mathrm{MHz}$, $\left.\mathrm{CDCl}_{3}\right) \delta 158.40,157.8,138.2,137.6,133.9,129.0,128.0,126.3,114.1,113.7,67.0,55.3,47.8,41.3$, 35.7, 29.7, 21.4. HRMS m/z: (APCI) [M-OH] ${ }^{+}$, calculated for $\mathrm{C}_{21} \mathrm{H}_{25} \mathrm{O}_{2} ; 309.1849$; found 309.1840 .<smiles>[Y6]C(C=O)C/C=C/[C@H](C)c1ccc(Cl)cc1</smiles>

(2R* $\left.{ }^{*}, 6 \mathrm{~S}^{*}, \mathrm{E}\right)-6$-(4-chlorophenyl)-2-methylhept-4-enal $2 \mathrm{r}$ (56\% yield): ${ }^{1} \mathrm{H} \mathrm{NMR}\left(400 \mathrm{MHz}, \mathrm{CDCl}_{3}\right) \delta 9.57$ (d, J $=1.2 \mathrm{~Hz}, 1 \mathrm{H}), 7.21-7.17(\mathrm{~m}, 2 \mathrm{H}), 7.04(\mathrm{~d}, \mathrm{~J}=8.4 \mathrm{~Hz}, 2 \mathrm{H}), 5.56(\mathrm{dd}, \mathrm{J}=15.2,6.7 \mathrm{~Hz}, 1 \mathrm{H}), 5.31(\mathrm{dt}, \mathrm{J}=15.1$, $3.6 \mathrm{~Hz}, 1 \mathrm{H}), 3.34(\mathrm{p}, \mathrm{J}=6.9 \mathrm{~Hz}, 1 \mathrm{H}), 2.41-2.30(\mathrm{~m}, 2 \mathrm{H}), 2.12-2.01(\mathrm{~m}, 1 \mathrm{H}), 1.24(\mathrm{~d}, \mathrm{~J}=7.0 \mathrm{~Hz}, 3 \mathrm{H}), 1.02$ $(\mathrm{d}, \mathrm{J}=6.8 \mathrm{~Hz}, 3 \mathrm{H}) .{ }^{13} \mathrm{C} \mathrm{NMR}\left(101 \mathrm{MHz}, \mathrm{CDCl}_{3}\right) \delta 204.7,144.3,137.6,131.8,128.5,125.6,46.3,41.7,33.5$, 21.3, 13.1. HRMS m/z: (APCl) [M-H] ${ }^{+}$, calculated for $\mathrm{C}_{14} \mathrm{H}_{16} \mathrm{ClO} ; 235.0884$; found 235.0876 .

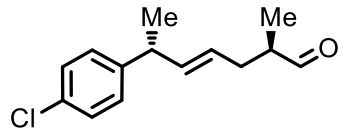

$\left(2 \mathrm{R}^{*}, 6 \mathrm{R}^{*}, \mathrm{E}\right)-6-(4-c h l o r o p h e n y l)-2-m e t h y l h e p t-4-e n a l ~ 2 s ~\left(53 \%\right.$ yield): ${ }^{1} \mathrm{H} \mathrm{NMR}\left(400 \mathrm{MHz}, \mathrm{CDCl}_{3}\right) \delta 9.57$ (d, J $=1.5 \mathrm{~Hz}, 1 \mathrm{H}), 7.20-7.16(\mathrm{~m}, 4 \mathrm{H}), 7.06-7.02(\mathrm{~m}, 3 \mathrm{H}), 5.56(\mathrm{ddt}, \mathrm{J}=7.8,6.7,1.2 \mathrm{~Hz}, 1 \mathrm{H}), 5.32$ (dtd, J = 15.2, 7.0, 1.3 Hz, 1H), $3.34(p, J=6.9 \mathrm{~Hz}, 1 \mathrm{H}), 2.40-2.31(\mathrm{~m}, 3 \mathrm{H}), 2.12-2.02(\mathrm{~m}, 1 \mathrm{H}), 1.24(\mathrm{~d}, \mathrm{~J}=7.0 \mathrm{~Hz}, 3 \mathrm{H})$, 
$1.18(\mathrm{~s}, 1 \mathrm{H}), 1.01$ (d, J = 6.9 Hz, 3H). ${ }^{13} \mathrm{C} N M R\left(101 \mathrm{MHz}, \mathrm{CDCl}_{3}\right) \delta 204.8,144.4,137.6,131.8,128.5,125.6$, 46.3, 41.7, 33.6, 21.3, 13.1. HRMS m/z: (APCl) [M-H] $]^{+}$, calculated for $\mathrm{C}_{14} \mathrm{H}_{16} \mathrm{ClO} ; 235.0884$; found 235.0893.<smiles></smiles>

$\left(2 \mathrm{R}^{*}, 6 \mathrm{~S}^{*}, \mathrm{E}\right)-6-(4-m e t h o x y p h e n y l)-2-m e t h y l h e p t-4-e n a l ~ 2 t ~\left(60 \%\right.$ yield): ${ }^{1} \mathrm{H} \mathrm{NMR}\left(400 \mathrm{MHz}, \mathrm{CDCl}_{3}\right) \delta 9.64$ (d, $\mathrm{J}=1.4 \mathrm{~Hz}, 1 \mathrm{H}), 7.13-7.08(\mathrm{~m}, 2 \mathrm{H}), 6.87-6.83(\mathrm{~m}, 2 \mathrm{H}), 5.66(\mathrm{dd}, \mathrm{J}=15.2,6.7 \mathrm{~Hz}, 1 \mathrm{H}), 5.42-5.33(\mathrm{~m}, 1 \mathrm{H})$, $3.79(\mathrm{~s}, 3 \mathrm{H}), 3.39(\mathrm{p}, \mathrm{J}=7.1 \mathrm{~Hz}, 1 \mathrm{H}), 2.42(\mathrm{qd}, \mathrm{J}=13.1,6.7 \mathrm{~Hz}, 2 \mathrm{H}), 2.18-2.07(\mathrm{~m}, 1 \mathrm{H}), 1.31(\mathrm{~d}, \mathrm{~J}=7.0 \mathrm{~Hz}$, 3H), 1.09 (d, J = 6.9 Hz, 3H). ${ }^{13} \mathrm{C} \mathrm{NMR} \mathrm{(101} \mathrm{MHz,} \mathrm{CDCl} 3$ ) $\delta$ 205.0, 158.0, 138.6, 138.1, 128.2, 124.9, 113.9, $77.5,77.2,76.8,55.4,46.5,41.5,33.7,29.8,21.6,13.2$. HRMS m/z: (APCI) $[\mathrm{M}+\mathrm{H}]^{+}$, calculated for $\mathrm{C}_{15} \mathrm{H}_{21} \mathrm{O} 2$; 233.1536; found 233.1514 .<smiles>COc1ccc(C(C)(C)CCC(C)C=O)cc1</smiles>

$\left(2 \mathrm{R}^{*}, 6 \mathrm{R}^{*}, \mathrm{E}\right)-6-(4-m e t h o x y p h e n y l)-2-m e t h y l h e p t-4-e n a l ~ 2 \mathrm{u}\left(60 \%\right.$ yield): ${ }^{1} \mathrm{H} \mathrm{NMR}\left(400 \mathrm{MHz}, \mathrm{CDCl}_{3}\right) \delta 9.64$ $(\mathrm{d}, \mathrm{J}=1.5 \mathrm{~Hz}, 1 \mathrm{H}), 7.13-7.08(\mathrm{~m}, 3 \mathrm{H}), 6.86-6.82(\mathrm{~m}, 3 \mathrm{H}), 5.66$ (ddd, J = 16.3, 6.8, $1.2 \mathrm{~Hz}, 1 \mathrm{H}$ ), 5.38 (dtd, $\mathrm{J}=15.2,6.9,1.3 \mathrm{~Hz}, 1 \mathrm{H}), 3.79(\mathrm{~s}, 3 \mathrm{H}), 3.39(\mathrm{p}, \mathrm{J}=7.0 \mathrm{~Hz}, 1 \mathrm{H}), 2.46-2.38(\mathrm{~m}, 3 \mathrm{H}), 2.18-2.09(\mathrm{~m}, 1 \mathrm{H}), 1.31$ $(\mathrm{d}, \mathrm{J}=7.0 \mathrm{~Hz}, 3 \mathrm{H}), 1.08$ (d, J = 6.8 Hz, 3H). ${ }^{13} \mathrm{C} \mathrm{NMR} \mathrm{(101} \mathrm{MHz,} \mathrm{CDCl} 3$ ) $\delta$ 204.9, 157.9, 138.5, 138.0, 128.0, $124.8,113.8,55.3,46.3,41.4,33.6,21.5,13.1$. HRMS m/z: (APCl) $[\mathrm{M}-\mathrm{H}]^{+}$, calculated for $\mathrm{C}_{15} \mathrm{H}_{19} \mathrm{O} 2$; 231.1380; found 231.1406.

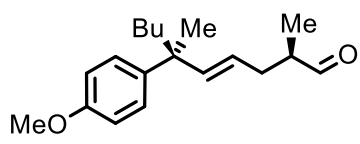

$\left(2 \mathrm{R}^{*}, 6 \mathrm{~S}^{*}, \mathrm{E}\right)-6$-(4-methoxyphenyl)-2,6-dimethyldec-4-enal $2 \mathbf{v}$ (52\% yield): ${ }^{1} \mathrm{H} \mathrm{NMR}\left(400 \mathrm{MHz}, \mathrm{CDCl}_{3}\right) \delta 9.66$ $(\mathrm{d}, \mathrm{J}=1.4 \mathrm{~Hz}, 1 \mathrm{H}), 7.21-7.16(\mathrm{~m}, 2 \mathrm{H}), 6.86-6.81(\mathrm{~m}, 2 \mathrm{H}), 5.68(\mathrm{~d}, \mathrm{~J}=15.6 \mathrm{~Hz}, 1 \mathrm{H}), 5.37-5.28(\mathrm{~m}, 1 \mathrm{H})$, $3.79(\mathrm{~s}, 3 \mathrm{H}), 2.52-2.39(\mathrm{~m}, 2 \mathrm{H}), 2.21-2.13(\mathrm{~m}, 1 \mathrm{H}), 1.77-1.62(\mathrm{~m}, 2 \mathrm{H}), 1.27-1.24(\mathrm{~m}, 4 \mathrm{H}), 1.10(\mathrm{~d}, \mathrm{~J}=$ $6.9 \mathrm{~Hz}, 3 \mathrm{H}), 0.85(\mathrm{t}, \mathrm{J}=7.3 \mathrm{~Hz}, 4 \mathrm{H}) .{ }^{13} \mathrm{C} \mathrm{NMR}\left(101 \mathrm{MHz}, \mathrm{CDCl}_{3}\right) \delta 205.0,157.5,142.6,140.1,127.6,123.0$, $113.4,55.2,46.5,43.0,41.5,34.0,26.8,25.8,23.4,14.1,13.1$. HRMS m/z: (APCI) $[\mathrm{M}+\mathrm{H}]^{+}$, calculated for $\mathrm{C}_{19} \mathrm{H}_{29} \mathrm{O} 2 ; 289.2162$; found 289.2156 .<smiles>COc1ccc(C(C)(C)C=CCC(O)c2ccccc2)cc1</smiles>

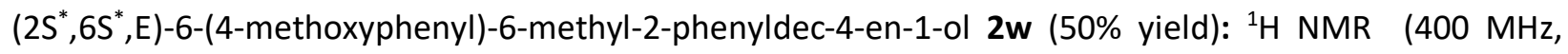
$\left.\mathrm{CDCl}_{3}\right) \delta 7.29-7.23(\mathrm{~m}, 2 \mathrm{H}), 7.17(\mathrm{t}, \mathrm{J}=2.2 \mathrm{~Hz}, 1 \mathrm{H}), 7.16-7.12(\mathrm{~m}, 2 \mathrm{H}), 6.99-6.94(\mathrm{~m}, 2 \mathrm{H}), 6.72-6.67$ $(\mathrm{m}, 2 \mathrm{H}), 5.46(\mathrm{~d}, \mathrm{~J}=15.7 \mathrm{~Hz}, 1 \mathrm{H}), 5.21(\mathrm{dt}, \mathrm{J}=15.6,7.0 \mathrm{~Hz}, 1 \mathrm{H}), 3.73(\mathrm{dd}, \mathrm{J}=6.7,4.4 \mathrm{~Hz}, 2 \mathrm{H}), 3.71(\mathrm{~s}, 3 \mathrm{H})$, $3.69(\mathrm{dd}, \mathrm{J}=6.6,4.3 \mathrm{~Hz}, 1 \mathrm{H}), 2.88-2.79(\mathrm{~m}, 1 \mathrm{H}), 2.46-2.38(\mathrm{~m}, 1 \mathrm{H}), 2.33-2.24(\mathrm{~m}, 1 \mathrm{H}), 1.55-1.41(\mathrm{~m}$, 4H), $1.15(\mathrm{~s}, 3 \mathrm{H}), 1.11(\mathrm{dd}, \mathrm{J}=14.8,7.4 \mathrm{~Hz}, 3 \mathrm{H}), 0.73(\mathrm{t}, \mathrm{J}=7.3 \mathrm{~Hz}, 3 \mathrm{H}) \cdot{ }^{13} \mathrm{C} \mathrm{NMR}\left(101 \mathrm{MHz}, \mathrm{CDCl}_{3}\right) \delta 157.3$, 
142.0, 141.5, 140.4, 128.7, 128.2, 127.6, 126.8, 124.3, 113.2, 67.1, 55.2, 48.9, 42.9, 41.5, 35.9, 26.7, 25.8, 23.4, 14.0. HRMS m/z: (APCI) [M-H] $]^{+}$, calculated for $\mathrm{C}_{24} \mathrm{H}_{31} \mathrm{O}_{2} ; 335.2369$; found 335.2392.

\section{Procedure for the preparation of alkenyltriflates}

All alkenyl triflates of aldehydes were prepared from literature reports and analytic data were in perfect agreement with the one reported. ${ }^{9}$ methyl (Z)-3-(((trifluoromethyl)sulfonyl)oxy)but-2-enoate was prepared from methyl acetoacetate according to another previous report in perfect agreement with the experimental data. ${ }^{10,11}$

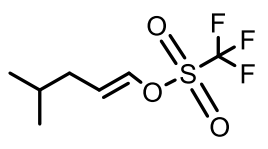

(E)-4-Methylpent-1-en-1-yl trifluoromethanesulfonate $(E: Z=6: 1):{ }^{1} \mathrm{H} \mathrm{NMR}\left(400 \mathrm{MHz}, \mathrm{CDCl}_{3}\right) \delta 6.58(\mathrm{~d}, \mathrm{~J}=$ $5.7 \mathrm{~Hz}, 6 \mathrm{H}), 5.26(\mathrm{td}, \mathrm{J}=7.7,5.8 \mathrm{~Hz}, 6 \mathrm{H}), 2.13-2.05(\mathrm{~m}, 12 \mathrm{H}), 1.70(\mathrm{dp}, \mathrm{J}=13.3,6.6 \mathrm{~Hz}, 7 \mathrm{H}), 0.93(\mathrm{~d}, \mathrm{~J}=$ $6.6 \mathrm{~Hz}, 35 \mathrm{H}) .{ }^{13} \mathrm{CNMR}\left(101 \mathrm{MHz}, \mathrm{CDCl}_{3}\right) \delta 135.7,119.4,35.5,34.2,33.0,27.9,22.4,22.1,22.0,14.1$. HRMS $\mathrm{m} / \mathrm{z}$ : (ESI) [M-H] $]^{-}$c calculated for $\mathrm{C}_{7} \mathrm{H}_{10} \mathrm{~F}_{3} \mathrm{O}_{3} \mathrm{~S} ; 231.0303$; found 231.0288 .

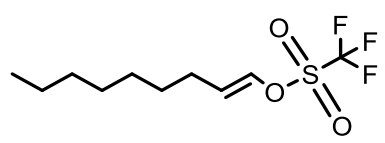

(E)-4-Methylpent-1-en-1-yl trifluoromethanesulfonate $(E: Z=4.5: 1):{ }^{1} \mathrm{H} \mathrm{NMR}\left(400 \mathrm{MHz}, \mathrm{CDCl}_{3}\right) \delta 6.52$ (d, J $=5.7 \mathrm{~Hz}, 1 \mathrm{H}), 5.25(\mathrm{td}, \mathrm{J}=7.6,5.7 \mathrm{~Hz}, 1 \mathrm{H}), 2.19(\mathrm{qd}, \mathrm{J}=7.5,1.5 \mathrm{~Hz}, 2 \mathrm{H}), 1.43-1.37(\mathrm{~m}, 2 \mathrm{H}), 1.33-1.25$ (m, 9H), 0.88 (s, 2H). ${ }^{13} \mathrm{C} \mathrm{NMR}\left(101 \mathrm{MHz}, \mathrm{CDCl}_{3}\right) \delta 135.22,123.01,120.96,120.26,117.08,77.38,77.06$, 76.74, 31.77, 29.04, 29.00, 28.58, 24.17, 22.65, 14.09. HRMS m/z: (APCl) $[\mathrm{M}+\mathrm{H}]^{+}$, calculated for $\mathrm{C}_{10} \mathrm{H}_{18} \mathrm{~F}_{3} \mathrm{O}_{3} \mathrm{~S} ; 275.0929$; found 275.0923 .

\section{General procedure for the tandem Heck alkenylation - ring opening with alkenyltriflates (Scheme 4)}

In a dry one-neck flask under $\mathrm{Ar}$, were added 5-(2-pyridyl)-1,3-oxazole (16 mol\%), $\mathrm{Pd}_{2} \mathrm{dba}_{3}$ (4 mol\%, molecular sieves $3 \AA$ ( $100 \% \mathrm{w} / \mathrm{w}$ substrate) and vinylcyclopropyl carbinol 1 (1 equiv., $0.2 \mathrm{mmol}$ for all reactions, unless otherwise noticed) in DMA ( $8 \mathrm{~mL} / \mathrm{mmol}$ substrate). The corresponding alkenyl triflate (1.5-2 equiv.) was then rapidly added and the mixture was heated to $55{ }^{\circ} \mathrm{C}$ and stirred for $18 \mathrm{~h}$. After completion of the reaction, the reaction mixture was diluted with brine and EtOAc. The phases were separated and the aqueous phase was successively extracted three times with EtOAc. The combined organic phases were then dried over anhydrous $\mathrm{MgSO}_{4}$ and concentrated under reduced pressure. The crude mixture was dissolved in $5 \mathrm{ml}$ of methanol and $\mathrm{NaBH}_{4}$ (2equiv) was added at $0{ }^{\circ} \mathrm{C}$. The mixture was stirred for an hour at room temperature and quenched with an aqueous solution of $\mathrm{HCl} 1 \mathrm{M}(10 \mathrm{ml})$. After completion of the reaction, the reaction mixture was diluted with brine and EtOAc. The phases were separated and the aqueous phase was successively extracted three times with EtOAc. The combined organic phases were then dried over anhydrous $\mathrm{MgSO}_{4}$ and concentrated under reduced pressure. Purification of the crude reaction mixture by column chromatography afforded the desired product 4. 


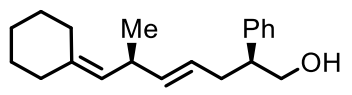

$\left(2 \mathrm{~S}^{*}, 6 \mathrm{~S}^{*}, \mathrm{E}\right)-7-c y c l o h e x y l i d e n e-6-m e t h y l-2-p h e n y l h e p t-4-e n-1-o l ~ 4 a\left(60 \%\right.$ yield): ${ }^{1} \mathrm{H} \mathrm{NMR}\left(400 \mathrm{MHz}, \mathrm{CDCl}_{3}\right.$ ) $\delta 7.32(\mathrm{t}, \mathrm{J}=7.3 \mathrm{~Hz}, 2 \mathrm{H}), 7.21(\mathrm{dd}, \mathrm{J}=8.4,7.0 \mathrm{~Hz}, 3 \mathrm{H}), 5.37$ (dd, J = 15.4, $6.1 \mathrm{~Hz}, 1 \mathrm{H}), 5.32-5.23(\mathrm{~m}, 1 \mathrm{H})$, $4.83(\mathrm{~d}, \mathrm{~J}=8.9 \mathrm{~Hz}, 1 \mathrm{H}), 3.75(\mathrm{dd}, \mathrm{J}=20.8,13.1 \mathrm{~Hz}, 2 \mathrm{H}), 3.06-2.94(\mathrm{~m}, 1 \mathrm{H}), 2.91-2.79(\mathrm{~m}, 1 \mathrm{H}), 2.44-2.26$ $(\mathrm{m}, 2 \mathrm{H}), 2.09-2.00(\mathrm{~m}, 4 \mathrm{H}), 1.54-1.43(\mathrm{~m}, 6 \mathrm{H}), 0.94(\mathrm{~d}, \mathrm{~J}=6.8 \mathrm{~Hz}, 3 \mathrm{H}) .{ }^{13} \mathrm{C} \mathrm{NMR}\left(101 \mathrm{MHz}, \mathrm{CDCl}_{3}\right) \delta 142.3$, 138.8, 137.8, 128.6, 128.1, 126.7, 125.7, 125.1, 67.0, 48.6, 37.2, 35.7, 34.4, 29.0, 28.7, 27.9, 26.9, 21.6. HRMS m/z: (APCI) $[\mathrm{M}+\mathrm{H}]^{+}$, calculated for $\mathrm{C}_{20} \mathrm{H}_{29} \mathrm{O} ; 285.2213$; found 285.2213.

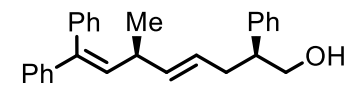

(2S* 6S*,E)-6-methyl-2,8,8-triphenylocta-4,7-dien-1-ol 4b (63\% yield): ${ }^{1} \mathrm{H}$ NMR (400 MHz, $\left.\mathrm{CDCl}_{3}\right) \delta 7.36$ $7.26(\mathrm{~m}, 6 \mathrm{H}), 7.21$ (dddd, J = 9.8, 7.9, 4.3, $1.6 \mathrm{~Hz}, 7 \mathrm{H}), 7.15-7.07(\mathrm{~m}, 2 \mathrm{H}), 5.80(\mathrm{~d}, \mathrm{~J}=10.1 \mathrm{~Hz}, 1 \mathrm{H}), 5.42$ (dd, J = 15.4, 5.9 Hz, 1H), $5.32-5.22(\mathrm{~m}, 1 \mathrm{H}), 3.78$ (ddd, J = 18.4, 10.9, 6.7 Hz, 2H), 2.87 (tt, J = 14.1, 7.0 $\mathrm{Hz}, 1 \mathrm{H}), 2.42(\mathrm{dt}, \mathrm{J}=14.0,7.0 \mathrm{~Hz}, 1 \mathrm{H}), 2.37-2.26(\mathrm{~m}, 1 \mathrm{H}), 1.01(\mathrm{~d}, \mathrm{~J}=6.8 \mathrm{~Hz}, 3 \mathrm{H}) .{ }^{13} \mathrm{C} \mathrm{NMR}(101 \mathrm{MHz}$, $\left.\mathrm{CDCl}_{3}\right) \delta 142.7,142.1,140.3,136.8,133.6,129.8,128.8,128.3,128.2,128.2,127.4,127.0,126.9,126.3$, 67.1, 48.9, 36.8, 35.7, 29.8, 21.3. HRMS m/z: (APCI) $[\mathrm{M}+\mathrm{H}]^{+}$, calculated for $\mathrm{C}_{27} \mathrm{H}_{29} \mathrm{O}$; 369.2213; found 369.2206 .

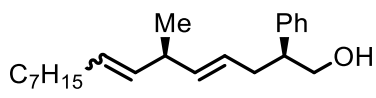

$\left(2 \mathrm{~S}^{*}, 4 \mathrm{E}, 6 \mathrm{R}^{*}\right)$-6-methyl-2-phenylpentadeca-4,7-dien-1-ol 4c (57\% yield): ${ }^{1} \mathrm{H} \mathrm{NMR}\left(400 \mathrm{MHz}, \mathrm{CDCl}_{3}\right) \delta 7.32$ $(\mathrm{t}, \mathrm{J}=7.5 \mathrm{~Hz}, 1 \mathrm{H}), 7.26-7.18(\mathrm{~m}, 1 \mathrm{H}), 5.41-5.24(\mathrm{~m}, 1 \mathrm{H}), 3.78(\mathrm{ddd}, \mathrm{J}=18.2,12.5,7.7 \mathrm{~Hz}, 1 \mathrm{H}), 2.91-2.79$ $(\mathrm{m}, 1 \mathrm{H}), 2.70(\mathrm{dd}, \mathrm{J}=11.6,6.0 \mathrm{~Hz}, 1 \mathrm{H}), 2.35(\mathrm{dtt}, \mathrm{J}=33.5,13.5,6.9 \mathrm{~Hz}, 1 \mathrm{H}), 2.05-1.90(\mathrm{~m}, 1 \mathrm{H}), 1.28(\mathrm{~d}, \mathrm{~J}$ $=15.4 \mathrm{~Hz}, 4 \mathrm{H}), 0.95(\mathrm{t}, \mathrm{J}=6.6 \mathrm{~Hz}, 1 \mathrm{H}), 0.88(\mathrm{t}, \mathrm{J}=6.7 \mathrm{~Hz}, 1 \mathrm{H}) .{ }^{13} \mathrm{C} \mathrm{NMR}\left(101 \mathrm{MHz}, \mathrm{CDCl}_{3}\right) \delta 142.2,137.3$, 137.1, 134.4, 133.8, 129.0, 128.9, 128.6, 128.1, 128.1, 126.8, 125.80, 125.5, 66.9, 66.9, 48.6, 39.3, 35.7, $34.6,32.6,31.9,29.8,29.6,29.3,29.2,29.2,29.2,27.4,22.7,21.2,20.6,14.2 . \mathrm{HRMS} \mathrm{m} / \mathrm{z}:(\mathrm{APCl})[\mathrm{M}+\mathrm{H}]^{+}$, calculated for $\mathrm{C}_{22} \mathrm{H}_{35} \mathrm{O} ; 315.2682$; found 315.2695 .

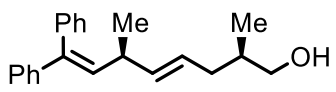

$\left(2 \mathrm{R}^{*}, 6 \mathrm{~S}^{*}, \mathrm{E}\right)-2,6$-dimethyl-8,8-diphenylocta-4,7-dien-1-ol 4d (68\% yield): ${ }^{1} \mathrm{H}$ NMR (400 MHz, $\left.\mathrm{CDCl}_{3}\right) \delta 7.32-$ $7.22(\mathrm{~m}, 1 \mathrm{H}$ ), 7.14 (ddd, J = 11.0, 10.3, $7.2 \mathrm{~Hz}, 2 \mathrm{H}$ ), 5.82 (d, J = $10.1 \mathrm{~Hz}, 1 \mathrm{H}$ ), 5.40 (dd, J = 15.4, $5.8 \mathrm{~Hz}, 1 \mathrm{H}$ ), $5.34-5.24(\mathrm{~m}, 1 \mathrm{H}), 3.40(\mathrm{ddd}, \mathrm{J}=27.0,10.6,6.2 \mathrm{~Hz}, 1 \mathrm{H}), 2.98-2.85(\mathrm{~m}, 1 \mathrm{H}), 2.08-2.00(\mathrm{~m}, 1 \mathrm{H}), 1.82(\mathrm{dt}$, $\mathrm{J}=13.9,7.1 \mathrm{~Hz}, 1 \mathrm{H}), 1.02(\mathrm{~d}, \mathrm{~J}=6.8 \mathrm{~Hz}, 1 \mathrm{H}), 0.83(\mathrm{~d}, \mathrm{~J}=6.8 \mathrm{~Hz}, 1 \mathrm{H}) .{ }^{13} \mathrm{C} \mathrm{NMR}\left(101 \mathrm{MHz}, \mathrm{CDCl}_{3}\right) \delta 142.7$, 140.4, 140.3, 136.4, 133.7, 129.9, 128.3, 128.2, 127.4, 127.1, 127.0, 126.8, 77.5, 77.2, 76.8, 68.2, 36.9, 36.7, 36.1, 29.8, 21.5, 16.6, 1.2. HRMS m/z: (APCl) $[\mathrm{M}-\mathrm{H}]^{+}$, calculated for $\mathrm{C}_{22} \mathrm{H}_{25} \mathrm{O}$; 305.1900; found 305.1921.

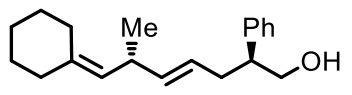


$\left(2 \mathrm{~S}^{*}, 6 \mathrm{R}^{*}, \mathrm{E}\right)-7-c y c l o h e x y l i d e n e-6-m e t h y l-2-p h e n y l h e p t-4-e n-1-o l ~ 4 e\left(63 \%\right.$ yield): ${ }^{1} \mathrm{H} \mathrm{NMR}\left(400 \mathrm{MHz}, \mathrm{CDCl}_{3}\right)$ $\delta 7.25(\mathrm{t}, \mathrm{J}=7.4 \mathrm{~Hz}, 1 \mathrm{H}), 7.17-7.12(\mathrm{~m}, 1 \mathrm{H}), 5.32(\mathrm{dd}, \mathrm{J}=15.3,6.0 \mathrm{~Hz}, 1 \mathrm{H}), 5.20(\mathrm{dt}, \mathrm{J}=6.3,4.7 \mathrm{~Hz}, 1 \mathrm{H})$, $4.75(\mathrm{~d}, \mathrm{~J}=8.8 \mathrm{~Hz}, 1 \mathrm{H}), 3.78-3.69(\mathrm{~m}, 1 \mathrm{H}), 3.69-3.61(\mathrm{~m}, 1 \mathrm{H}), 2.92(\mathrm{dd}, \mathrm{J}=14.7,6.9 \mathrm{~Hz}, 1 \mathrm{H}), 2.81-2.71$ $(\mathrm{m}, 1 \mathrm{H}), 2.36-2.18(\mathrm{~m}, 1 \mathrm{H}), 1.97(\mathrm{dd}, \mathrm{J}=12.7,6.2 \mathrm{~Hz}, 1 \mathrm{H}), 1.42(\mathrm{dd}, \mathrm{J}=8.3,5.5 \mathrm{~Hz}, 2 \mathrm{H}), 0.89(\mathrm{~d}, \mathrm{~J}=6.8 \mathrm{~Hz}$, 1H). ${ }^{13} \mathrm{C} \mathrm{NMR}\left(101 \mathrm{MHz}, \mathrm{CDCl}_{3}\right) \delta 142.3,138.8,137.8,128.6,128.1,126.7,125.6,125.1,66.9,48.7,37.2$, 35.6, 34.3, 29.1, 28.7, 27.9, 26.9, 21.6. HRMS m/z: (APCI) $[\mathrm{M}+\mathrm{H}]^{+}$, calculated for $\mathrm{C}_{20} \mathrm{H}_{29} \mathrm{O} ; 285.2213$; found 285.2255 .

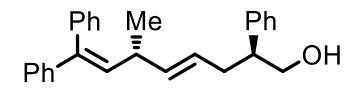

$\left(2 \mathrm{~S}^{*}, 6 \mathrm{R}^{*}, \mathrm{E}\right)-6-\mathrm{methyl}-2,8,8-$ triphenylocta-4,7-dien-1-ol $4 \mathrm{f}$ (65\% yield): ${ }^{1} \mathrm{H} \mathrm{NMR}\left(400 \mathrm{MHz}, \mathrm{CDCl}_{3}\right) \delta 7.36-$ $7.28(\mathrm{~m}, 6 \mathrm{H}), 7.23-7.18(\mathrm{~m}, 7 \mathrm{H}), 7.14-7.08(\mathrm{~m}, 2 \mathrm{H}), 5.79(\mathrm{~d}, \mathrm{~J}=10.1 \mathrm{~Hz}, 1 \mathrm{H}), 5.43(\mathrm{dd}, \mathrm{J}=15.4,6.0 \mathrm{~Hz}$, $1 \mathrm{H}), 5.28(\mathrm{dt}, \mathrm{J}=14.1,6.9 \mathrm{~Hz}, 1 \mathrm{H}), 3.78(\mathrm{ddd}, \mathrm{J}=18.4,10.8,6.6 \mathrm{~Hz}, 2 \mathrm{H}), 2.93-2.80(\mathrm{~m}, 2 \mathrm{H}), 2.41(\mathrm{dt}, \mathrm{J}=$ 14.4, 7.2 Hz, 1H), $2.36-2.28(\mathrm{~m}, 1 \mathrm{H}), 1.02(\mathrm{~d}, \mathrm{~J}=6.8 \mathrm{~Hz}, 3 \mathrm{H}) .{ }^{13} \mathrm{C} \mathrm{NMR}\left(101 \mathrm{MHz}, \mathrm{CDCl}_{3}\right) \delta$ 142.7, 142.1, 140.3, 136.8, 133.6, 129.8, 128.8, 128.3, 128.2, 128.2, 127.4, 127.0, 126.9, 126.3, 67.1, 48.9, 36.8, 35.7, 29.8, 21.3. HRMS m/z: (APCI) [M+H] $]^{+}$, calculated for $\mathrm{C}_{27} \mathrm{H}_{29} \mathrm{O}$; 369.2213; found 369.2210.<smiles>CCC=CC(C)C=CCCC(CO)c1ccccc1</smiles>

$\left(2 \mathrm{~S}^{*}, 4 \mathrm{E}, 6 \mathrm{~S}^{*}\right)$-6-methyl-2-phenylpentadeca-4,7-dien-1-ol $4 \mathrm{~g}$ (57\% yield): ${ }^{1} \mathrm{H}$ NMR (400 MHz, $\left.\mathrm{CDCl}_{3}\right) \delta 7.33$ $(\mathrm{td}, \mathrm{J}=7.5,1.5 \mathrm{~Hz}, 2 \mathrm{H}), 7.25-7.18(\mathrm{~m}, 3 \mathrm{H}), 5.52-5.07(\mathrm{~m}, 4 \mathrm{H}), 3.87-3.69(\mathrm{~m}, 2 \mathrm{H}), 3.52-3.38(\mathrm{~m}, 1 \mathrm{H})$, $3.15-3.03(\mathrm{~m}, 1 \mathrm{H}), 2.91-2.80(\mathrm{~m}, 1 \mathrm{H}), 2.62-2.47(\mathrm{~m}, 1 \mathrm{H}), 2.45-2.27(\mathrm{~m}, 1 \mathrm{H}), 2.08-1.92(\mathrm{~m}, 2 \mathrm{H}), 1.34$ $-1.23(\mathrm{~m}, 12 \mathrm{H}), 1.04-0.93(\mathrm{~m}, 3 \mathrm{H}), 0.89(\mathrm{~d}, \mathrm{~J}=6.6 \mathrm{~Hz}, 3 \mathrm{H}) .{ }^{13} \mathrm{C} \mathrm{NMR}\left(101 \mathrm{MHz}, \mathrm{CDCl}_{3}\right) \delta$ 142.2, 142.1, $137.2,137.0,136.2,134.4,134.1,133.8,129.0,128.9,128.7,128.6,128.3,128.1,128.1,128.0,126.9$, $126.8,125.9,125.5,125.1,77.4,77.1,76.7,48.6,48.6,39.3,35.6,34.6,32.6,31.9,30.6,30.2,29.9,29.8$, 29.6, 29.4, 29.3, 29.3, 29.2, 29.2, 29.2, 27.6, 27.4, 22.7, 22.0, 21.2, 20.6, 14.2. HRMS m/z: (APCI) [M+H] ${ }^{+}$, calculated for $\mathrm{C}_{22} \mathrm{H}_{35} \mathrm{O} ; 315.2682$; found 315.2686 .

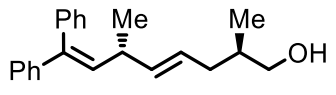

(2R $\left.{ }^{*}, 6 \mathrm{R}^{*}, \mathrm{E}\right)-2,6$-dimethyl-8,8-diphenylocta-4,7-dien-1-ol 4h (71\% yield): ${ }^{1} \mathrm{H} \mathrm{NMR}\left(400 \mathrm{MHz}, \mathrm{CDCl}_{3}\right) \delta 7.41$ $-7.30(\mathrm{~m}, 3 \mathrm{H}), 7.21$ (ddd, J = 11.4, 9.3, $6.0 \mathrm{~Hz}, 6 \mathrm{H}$ ), 5.89 (d, J = $10.1 \mathrm{~Hz}, 1 \mathrm{H}), 5.47$ (dd, J = 15.4, 5.8 Hz, 1H), $5.41-5.31(\mathrm{~m}, 1 \mathrm{H}), 3.51(\mathrm{dd}, \mathrm{J}=10.6,6.1 \mathrm{~Hz}, 1 \mathrm{H}), 3.44(\mathrm{dd}, \mathrm{J}=10.6,6.2 \mathrm{~Hz}, 1 \mathrm{H}), 3.04-2.93(\mathrm{~m}, 1 \mathrm{H}), 2.10$ $(\mathrm{dt}, \mathrm{J}=13.2,6.5 \mathrm{~Hz}, 1 \mathrm{H}), 1.91(\mathrm{dd}, \mathrm{J}=14.0,7.0 \mathrm{~Hz}, 1 \mathrm{H}), 1.69(\mathrm{dt}, \mathrm{J}=19.7,6.5 \mathrm{~Hz}, 2 \mathrm{H}), 1.09$ (d, J = $6.8 \mathrm{~Hz}$, $3 \mathrm{H}), 0.91$ (d, J = 6.8 Hz, 3H). ${ }^{13} \mathrm{C} \mathrm{NMR}\left(101 \mathrm{MHz}, \mathrm{CDCl}_{3}\right) \delta 142.7,140.4,140.3,136.5,133.8,129.9,128.3$, 128.2, 127.4, 127.1, 127.0, 126.8, 77.5, 77.2, 76.8, 68.1, 36.9, 36.7, 36.1, 29.8, 21.5, 16.6. HRMS m/z: (APCl) $[\mathrm{M}-\mathrm{H}]^{+}$, calculated for $\mathrm{C}_{22} \mathrm{H}_{25} \mathrm{O} ; 305.1900$; found 305.1924.

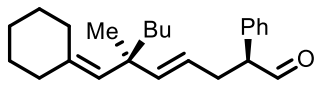


$\left(2 \mathrm{~S}^{*}, 6 \mathrm{~S}^{*}, \mathrm{E}\right)-6$-(cyclohexylidenemethyl)-6-methyl-2-phenyldec-4-enal 4i (56\% yield): ${ }^{1} \mathrm{H}$ NMR (400 MHz, $\left.\mathrm{CDCl}_{3}\right) \delta 9.71-9.68(\mathrm{~m}, 1 \mathrm{H}), 7.36(\mathrm{t}, \mathrm{J}=7.4 \mathrm{~Hz}, 2 \mathrm{H}), 7.29(\mathrm{~d}, \mathrm{~J}=6.9 \mathrm{~Hz}, 1 \mathrm{H}), 7.21-7.16(\mathrm{~m}, 2 \mathrm{H}), 5.47(\mathrm{~d}, \mathrm{~J}$ $=5.2 \mathrm{~Hz}, 1 \mathrm{H}), 5.22(\mathrm{ddd}, \mathrm{J}=15.6,13.0,6.8 \mathrm{~Hz}, 1 \mathrm{H}), 4.99(\mathrm{~s}, 1 \mathrm{H}), 3.61-3.53(\mathrm{~m}, 1 \mathrm{H}), 2.81(\mathrm{dt}, \mathrm{J}=14.0,6.8$ $\mathrm{Hz}, 1 \mathrm{H}), 2.53-2.42(\mathrm{~m}, 1 \mathrm{H}), 2.04-1.94(\mathrm{~m}, 4 \mathrm{H}), 1.48(\mathrm{~d}, \mathrm{~J}=2.9 \mathrm{~Hz}, 4 \mathrm{H}), 1.37(\mathrm{~d}, \mathrm{~J}=3.6 \mathrm{~Hz}, 2 \mathrm{H}), 1.26(\mathrm{~d}, \mathrm{~J}$ $=4.4 \mathrm{~Hz}, 6 \mathrm{H}), 1.00(\mathrm{~d}, \mathrm{~J}=1.5 \mathrm{~Hz}, 3 \mathrm{H}), 0.85(\mathrm{dt}, \mathrm{J}=7.2,3.6 \mathrm{~Hz}, 3 \mathrm{H}) .{ }^{13} \mathrm{C} \mathrm{NMR}\left(101 \mathrm{MHz}, \mathrm{CDCl}_{3}\right) \delta 200.84$, $142.51,142.47,141.22,136.25,136.20,129.16,129.09,129.08,129.04,127.65,122.05,121.94,59.57$, 43.80, 43.72, 40.26, 38.48, 33.35, 30.09, 30.07, 29.86, 29.16, 27.58, 26.89, 26.79, 26.70, 26.64, 23.58, 14.28. HRMS m/z: (APCI) $[\mathrm{M}-\mathrm{OH}]^{+}$, (for alcohol product after reduction) calculated for $\mathrm{C}_{24} \mathrm{H}_{35} ; 323.2733$; found 323.2747 .

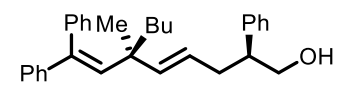

(2S* 6S*,E)-6-(2,2-diphenylvinyl)-6-methyl-2-phenyldec-4-en-1-ol 4j (61\% yield): ${ }^{1} \mathrm{H} \mathrm{NMR} \mathrm{(400} \mathrm{MHz,} \mathrm{CDCl} 3$ ) $\delta 7.33-7.27(\mathrm{~m}, 5 \mathrm{H}), 7.23-7.14(\mathrm{~m}, 8 \mathrm{H}), 7.08(\mathrm{dd}, \mathrm{J}=7.6,1.7 \mathrm{~Hz}, 2 \mathrm{H}), 5.93(\mathrm{~s}, 1 \mathrm{H}), 5.23(\mathrm{~d}, \mathrm{~J}=15.7 \mathrm{~Hz}$, $1 \mathrm{H}), 5.05(\mathrm{dt}, \mathrm{J}=15.6,6.9 \mathrm{~Hz}, 1 \mathrm{H}), 3.72(\mathrm{dt}, \mathrm{J}=18.9,8.7 \mathrm{~Hz}, 2 \mathrm{H}), 2.87-2.61(\mathrm{~m}, 2 \mathrm{H}), 2.23-2.14(\mathrm{~m}, 1 \mathrm{H})$, $2.09(\mathrm{dt}, \mathrm{J}=15.2,7.6 \mathrm{~Hz}, 1 \mathrm{H}), 1.28-1.21(\mathrm{~m}, 6 \mathrm{H}), 0.86(\mathrm{~s}, 3 \mathrm{H}), 0.83(\mathrm{t}, \mathrm{J}=7.1 \mathrm{~Hz}, 3 \mathrm{H}) .{ }^{13} \mathrm{C} \mathrm{NMR}(101 \mathrm{MHz}$, $\left.\mathrm{CDCl}_{3}\right) \delta 142.1,140.8,140.5,140.2,137.7,130.4,128.6,128.4,128.1,128.0,127.6,127.0,126.7,126.7$, 126.7, 123.5 67.1, 48.7, 43.6, 42.2, 35.7, 26.7, 25.4, 23.4, 14.1. HRMS m/z: (APCI) [M+H] ${ }^{+}$, calculated for $\mathrm{C}_{31} \mathrm{H}_{37} \mathrm{O} ; 425.2839$; found 425.2848 .

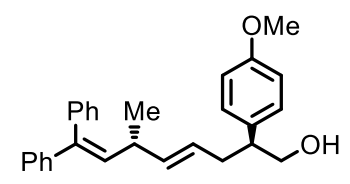

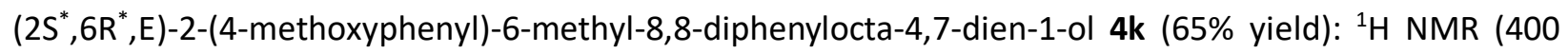
$\left.\mathrm{MHz}, \mathrm{CDCl}_{3}\right) \delta 7.38-7.27(\mathrm{~m}, 4 \mathrm{H}), 7.27-7.16(\mathrm{~m}, 6 \mathrm{H}), 7.15-7.10(\mathrm{~m}, 4 \mathrm{H}), 6.89-6.85(\mathrm{~m}, 2 \mathrm{H}), 5.81(\mathrm{~d}, \mathrm{~J}$ $=10.1 \mathrm{~Hz}, 1 \mathrm{H}$ ), $5.43(\mathrm{dd}, \mathrm{J}=15.4,6.1 \mathrm{~Hz}, 1 \mathrm{H}), 5.28$ (ddd, J = 15.2, 7.5, $1.1 \mathrm{~Hz}, 1 \mathrm{H}$ ), 3.80 (dd, J = 7.1, $5.6 \mathrm{~Hz}$, $1 \mathrm{H}), 3.77(\mathrm{~s}, 3 \mathrm{H}), 3.70(\mathrm{dd}, \mathrm{J}=10.8,7.7 \mathrm{~Hz}, 1 \mathrm{H}), 2.98-2.87(\mathrm{~m}, 1 \mathrm{H}), 2.86-2.76(\mathrm{~m}, 1 \mathrm{H}), 2.38(\mathrm{dt}, \mathrm{J}=14.3$, $7.2 \mathrm{~Hz}, 1 \mathrm{H}$ ), 2.28 (ddd, J = 14.1, 7.5, $6.7 \mathrm{~Hz}, 1 \mathrm{H}), 1.27(\mathrm{~s}, 1 \mathrm{H}), 1.04(\mathrm{~d}, \mathrm{~J}=6.8 \mathrm{~Hz}, 3 \mathrm{H}) .{ }^{13} \mathrm{C} \mathrm{NMR}(101 \mathrm{MHz}$, $\left.\mathrm{CDCl}_{3}\right) \delta 158.4,142.6,140.2,140.2,136.6,134.0,133.6,129.8,129.0,128.2,128.1,127.3,127.0,126.9$, $126.3,114.1,67.0,55.3,47.8,36.8,35.7,21.3$. HRMS m/z: (APCI) $[\mathrm{M}-\mathrm{OH}]^{+}$, calculated for $\mathrm{C}_{28} \mathrm{H}_{29} \mathrm{O}$; 381.2213; found 381.2209.

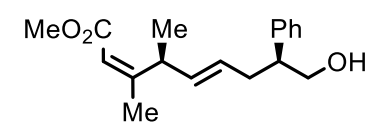

Methyl (2Z,4S* $\left.5 \mathrm{E}^{*}, 8 \mathrm{~S}\right)$-9-hydroxy-3,4-dimethyl-8-phenylnona-2,5-dienoate $4 \mathrm{I}$ (70\% yield): ${ }^{1} \mathrm{H}$ NMR (400 $\left.\mathrm{MHz}, \mathrm{CDCl}_{3}\right) \delta 7.35-7.28(\mathrm{~m}, 2 \mathrm{H}), 7.25-7.16(\mathrm{~m}, 3 \mathrm{H}), 5.64-5.60(\mathrm{~m}, 1 \mathrm{H}), 5.45-5.19(\mathrm{~m}, 1 \mathrm{H}), 3.82-3.70$ $(\mathrm{m}, 2 \mathrm{H}), 3.68(\mathrm{~s}, 3 \mathrm{H}), 2.91-2.74(\mathrm{~m}, 2 \mathrm{H}), 2.54-2.39(\mathrm{~m}, 1 \mathrm{H}), 2.37-2.27(\mathrm{~m}, 1 \mathrm{H}), 1.99(\mathrm{~d}, \mathrm{~J}=1.2 \mathrm{~Hz}, 3 \mathrm{H})$, $1.04(\mathrm{~d}, \mathrm{~J}=7.0 \mathrm{~Hz}, 3 \mathrm{H}) .{ }^{13} \mathrm{C}$ NMR $13 \mathrm{C}$ NMR $\left(101 \mathrm{MHz}, \mathrm{CDCl}_{3}\right) \delta 167.48,166.63,163.25,141.98,141.78$, $134.20,134.17,128.68,128.63,128.44,128.10,127.69,126.86,126.77,115.19,114.42,66.92,66.88$, 
50.88, 48.57, 46.29, 37.04, 35.64, 35.42, 19.88, 18.29, 17.66, 16.84. HRMS m/z: (APCI) $[\mathrm{M}+\mathrm{H}]^{+}$, calculated for $\mathrm{C}_{18} \mathrm{H}_{25} \mathrm{O}_{3} ; 289.1798$; found 289.1815 .

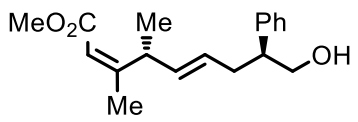

Methyl (2Z,4R* $\left.4 \mathrm{E}, 8 \mathrm{~S}^{*}\right)-9-$-hydroxy-3,4-dimethyl-8-phenylnona-2,5-dienoate $4 \mathrm{~m}$ (68\% yield): ${ }^{1} \mathrm{H}$ NMR $1 \mathrm{H}$ $\operatorname{NMR}\left(400 \mathrm{MHz}, \mathrm{CDCl}_{3}\right) \delta 7.31$ (ddd, J = 7.7, 3.1, 2.0 Hz, 2H), $7.25-7.16(\mathrm{~m}, 3 \mathrm{H}), 5.64-5.59(\mathrm{~m}, 1 \mathrm{H}), 5.42$ $-5.22(\mathrm{~m}, 2 \mathrm{H}), 3.83-3.70(\mathrm{~m}, 2 \mathrm{H}), 3.68(\mathrm{~d}, \mathrm{~J}=1.3 \mathrm{~Hz}, 3 \mathrm{H}), 2.90-2.74(\mathrm{~m}, 2 \mathrm{H}), 2.49-2.38(\mathrm{~m}, 1 \mathrm{H}), 2.35$ $-2.27(\mathrm{~m}, 1 \mathrm{H}), 1.97(\mathrm{~d}, \mathrm{~J}=1.2 \mathrm{~Hz}, 3 \mathrm{H}), 1.07(\mathrm{~d}, \mathrm{~J}=6.9 \mathrm{~Hz}, 3 \mathrm{H}) .{ }^{13} \mathrm{C} \mathrm{NMR}\left(101 \mathrm{MHz}, \mathrm{CDCl}_{3}\right) \delta$ 167.47, 166.62, 163.24, 142.03, 141.78, 134.25, 128.75, 128.69, 128.64, 128.56, 128.08, 128.02, 127.82, 126.87, 126.78, $115.19,114.34,66.87,66.84,50.87,48.57,46.34,37.10,35.66,35.41,19.97,18.44,17.99,16.87$. HRMS $\mathrm{m} / \mathrm{z}$ : (APCl) $[\mathrm{M}+\mathrm{H}]^{+}$, calculated for $\mathrm{C}_{18} \mathrm{H}_{25} \mathrm{O}_{3} ; 289.1798$; found 289.1813 .

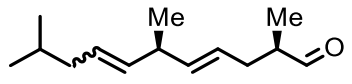

$\left(2 \mathrm{R}^{*}, 4 \mathrm{E}, 6 \mathrm{R}^{*}\right)-2,6,10$-trimethylundeca-4,7-dienal 4n (65\% yield): ${ }^{1} \mathrm{H} \mathrm{NMR} \quad\left(400 \mathrm{MHz}, \mathrm{CDCl}_{3}\right) \delta 9.64(\mathrm{dd}, \mathrm{J}=$ 3.0, $1.5 \mathrm{~Hz}, 1 \mathrm{H}), 5.50-5.17(\mathrm{~m}, 4 \mathrm{H}), 3.17-3.07(\mathrm{~m}, 1 \mathrm{H}), 2.77(\mathrm{dt}, \mathrm{J}=13.2,6.6 \mathrm{~Hz}, 1 \mathrm{H}), 2.49-2.33(\mathrm{~m}, 2 \mathrm{H})$, $2.18-2.04(\mathrm{~m}, 1 \mathrm{H}), 1.96-1.83(\mathrm{~m}, 2 \mathrm{H}), 1.58-1.54(\mathrm{~m}, 1 \mathrm{H}), 1.05$ (ddd, J = 18.3, 9.1, $5.2 \mathrm{~Hz}, 6 \mathrm{H}), 0.90-$ $0.85(\mathrm{~m}, 6 \mathrm{H}) .{ }^{13} \mathrm{C}$ NMR $\left(101 \mathrm{MHz}, \mathrm{CDCl}_{3}\right) \delta 205.0,138.1,137.8,135.4,134.2,127.8,127.6,124.4,124.1$, 77.3, 77.0, 76.7, 46.3, 41.9, 39.3, 36.5, 34.5, 33.6, 29.7, 28.6, 28.4, 22.4, 22.4, 22.2, 21.1, 20.6, 13.0, 12.9. HRMS m/z: (APCI) $[\mathrm{M}+\mathrm{H}]^{+}$, calculated for $\mathrm{C}_{14} \mathrm{H}_{25} \mathrm{O}$; 209.1900; found 209.1926.

\section{Procedure for the Hydrogenation of skipped diene 4n}

The hydrogenation of $\mathbf{4 n}$ to $\mathbf{5}$ was accomplished using a previously reported method for diimide reduction of olefins. ${ }^{12}$

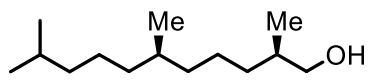

(2R* $\left.6 \mathrm{R}^{*}\right)$-2,6,10-trimethylundecan-1-ol 5 (86\% yield): ${ }^{1} \mathrm{H}$ NMR $\left(400 \mathrm{MHz}, \mathrm{CDCl}_{3}\right) \delta 3.55-3.47(\mathrm{~m}, 1 \mathrm{H}), 3.42$ (dd, J = 10.5, $6.5 \mathrm{~Hz}, 1 \mathrm{H}), 1.64-1.47(\mathrm{~m}, 3 \mathrm{H}), 1.26(\mathrm{~d}, \mathrm{~J}=9.9 \mathrm{~Hz}, 7 \mathrm{H}), 1.16-1.05(\mathrm{~m}, 5 \mathrm{H}), 0.92(\mathrm{~d}, \mathrm{~J}=6.7$ $\mathrm{Hz}, 3 \mathrm{H}), 0.86(\mathrm{t}, \mathrm{J}=6.9 \mathrm{~Hz}, 9 \mathrm{H}) .{ }^{13} \mathrm{C} \mathrm{NMR}\left(101 \mathrm{MHz}, \mathrm{CDCl}_{3}\right) \delta 77.4,77.1,76.7,68.4,39.4,37.4,37.3,35$. $33.5,32.8,29.8,28.0,24.8,24.4,22.7,22.7,19.8,19.7,16.7,16.6$. HRMS m/z: (APCI) $[M-H]^{+}$, calculated for $\mathrm{C}_{14} \mathrm{H}_{29} \mathrm{O} ; 213.2213$; found 213.2210. 


\section{References}

(1) Drent, E.; Van Broekhoven, J. A. M.; Doyle, M. J. Efficient Palladium Catalysts for the Copolymerization of Carbon Monoxide with Olefins to Produce Perfectly Alternating Polyketones. J. Organomet. Chem. 1991, 417, 235-251.

(2) Liu, X.; Fox, J. M. Enantioselective, Facially Selective Carbomagnesation of Cyclopropenes. J. Am. Chem. Soc. 2006, 128, 5600-5601.

(3) Liao, L. A.; Fox, J. M. A Copper-Catalyzed Method for the Facially Selective Addition of Grignard Reagents to Cyclopropenes. J. Am. Chem. Soc. 2002, 124, 14322-14323.

(4) Zhang, F. G.; Marek, I. Brook Rearrangement as Trigger for Carbene Generation: Synthesis of Stereodefined and Fully Substituted Cyclobutenes. J. Am. Chem. Soc. 2017, 139, 8364-8370.

(5) Brown, H. C.; Blue, C. D.; Nelson, D. J.; Bhat, N. G. Vinylic Organoboranes. 12. Synthesis of (Z)-1-Halo-1-Alkenes via Hydroboration of 1-Halo-l-Alkynes Followed by Protonolysis. J. Org. Chem. 1989, 54, 6064-6067.

(6) Müller, D.; Alexakis, A. Formation of Quaternary Stereogenic Centers by CopperCatalyzed Asymmetric Conjugate Addition Reactions of Alkenylaluminums to Trisubstituted Enones. Chem. - A Eur. J. 2013, 19, 15226-15239.

(7) Wipf, P.; Lim, S. Rapid Carboalumination of Alkynes in the Presence of Water. Angew. Chemie Int. Ed. English 1993, 32, 1068-1071.

(8) Zhang, H.; Huang, W.; Wang, T.; Meng, F. Cobalt-Catalyzed Diastereo- and Enantioselective Hydroalkenylation of Cyclopropenes with Alkenylboronic Acids. Angew. Chemie - Int. Ed. 2019, 58, 11049-11053.

(9) Wright, M. E.; Pulley, S. R. Vinyl Triflates: A Mechanistic Study on Their Formation from Carbonyl Compounds and Triflic Anhydride. J. Org. Chem. 1989, 54, 2886-2889.

(10) Chassaing, S.; Specklin, S.; Weibel, J. M.; Pale, P. Vinyl Triflates Derived from 1,3Dicarbonyl Compounds and Analogs: Access and Applications to Organic Synthesis. Tetrahedron 2012, 68, 7245-7273. 
(11) Specklin, S.; Bertus, P.; Weibel, J. M.; Pale, P. A Versatile and Highly Stereoselective Access to Vinyl Triflates Derived from 1,3-Dicarbonyl and Related Compounds. J. Org. Chem. 2008, 73, 7845-7848.

(12) Dandapani, S.; Jeske, M.; Curran, D. P. Synthesis of All 16 Stereoisomers of Pinesaw Fly Sex Pheromones - Tools and Tactics for Solving Problems in Fluorous Mixture Synthesis. J. Org. Chem. 2005, 70, 9447-9462. 


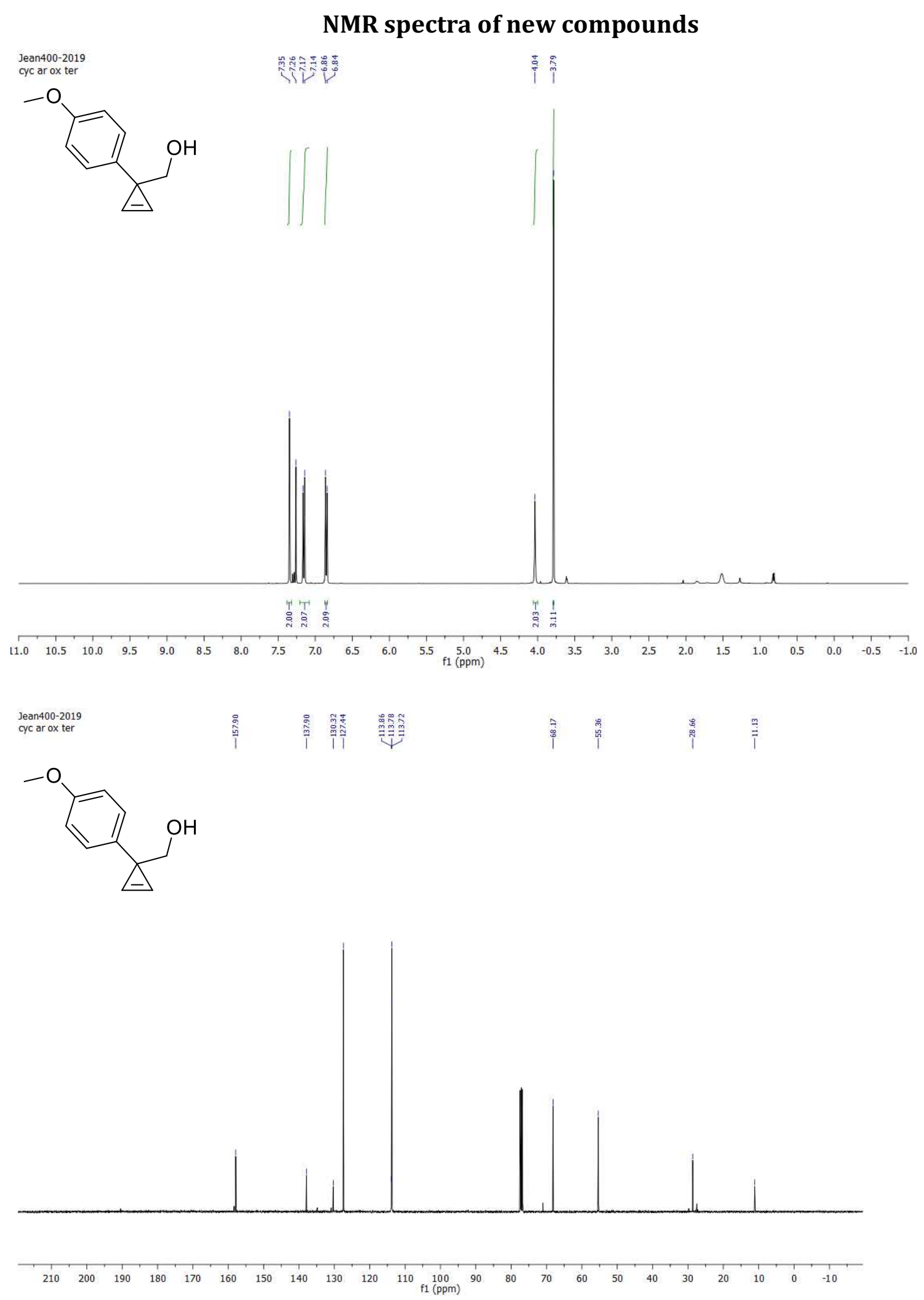


Anthony400-2019

$\Longrightarrow y_{\mathrm{OH}}^{\mathrm{D}}$
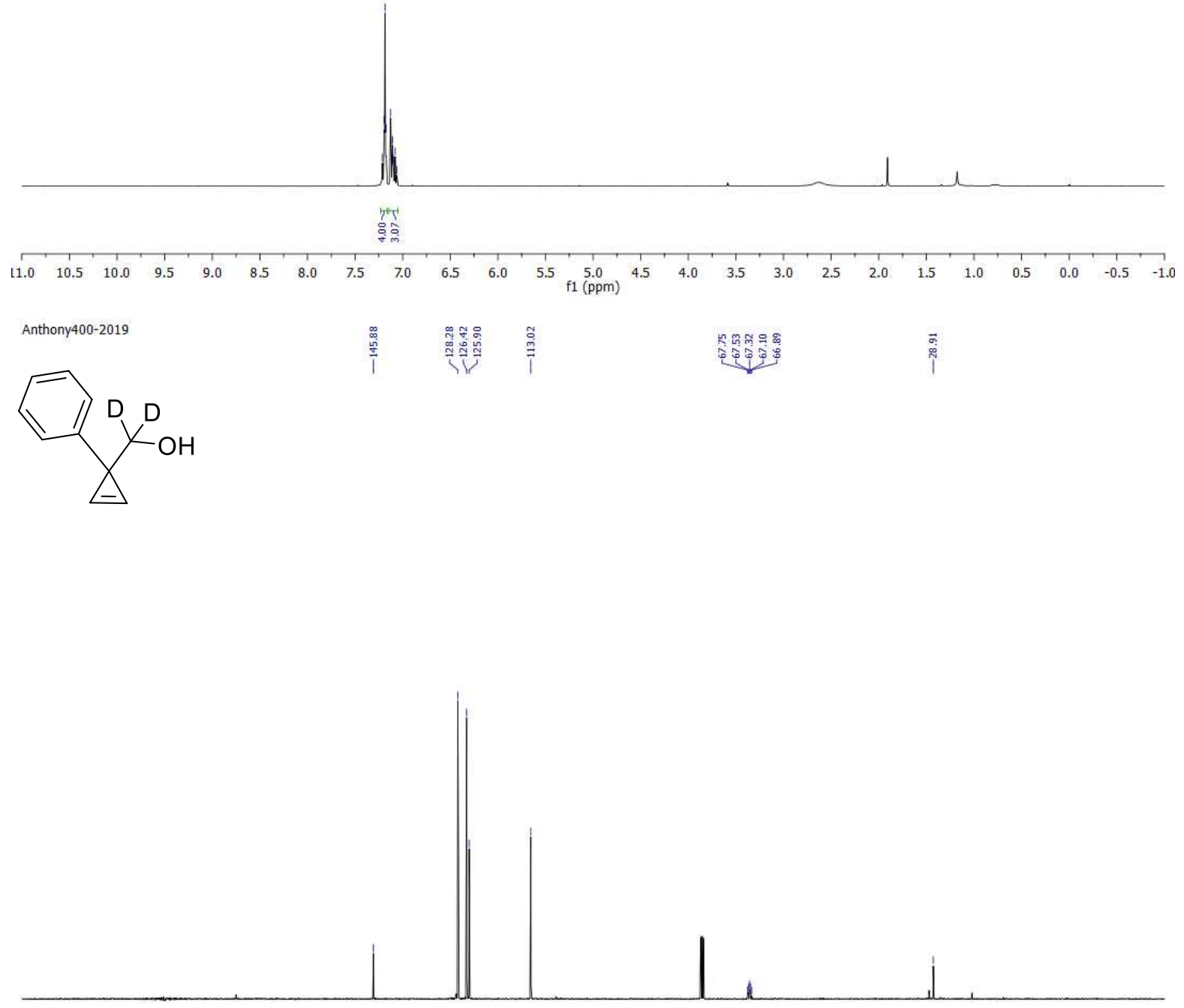

$\begin{array}{llllllllllllllllllllllll} & 210 & 200 & 190 & 180 & 170 & 160 & 150 & 140 & 130 & 120 & 110 & 100 & 90 & 80 & 70 & 60 & 50 & 40 & 30 & 20 & 10 & 0 & -10\end{array}$ 

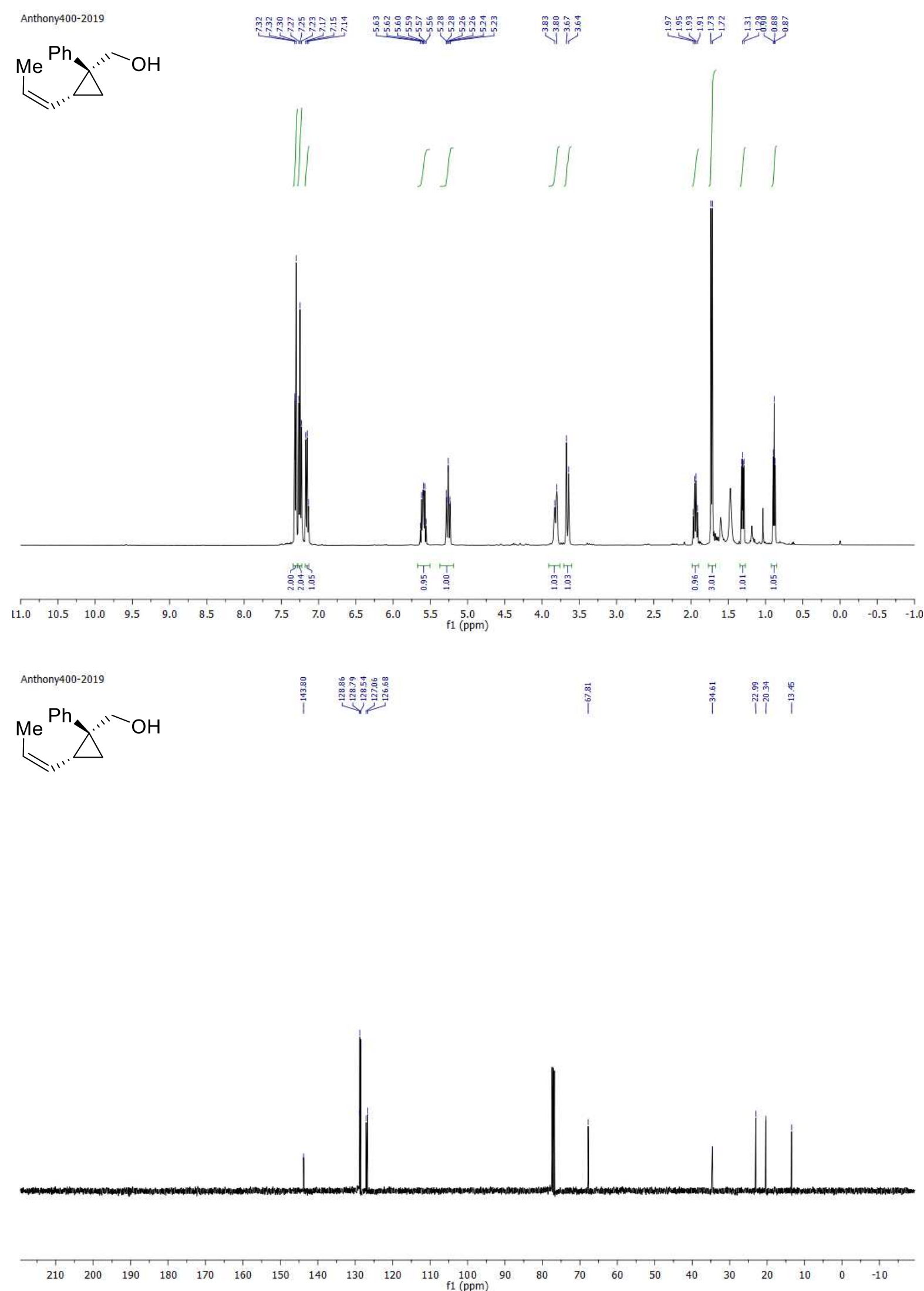
Anthony400-2019

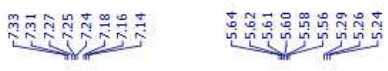

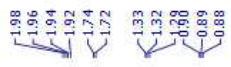
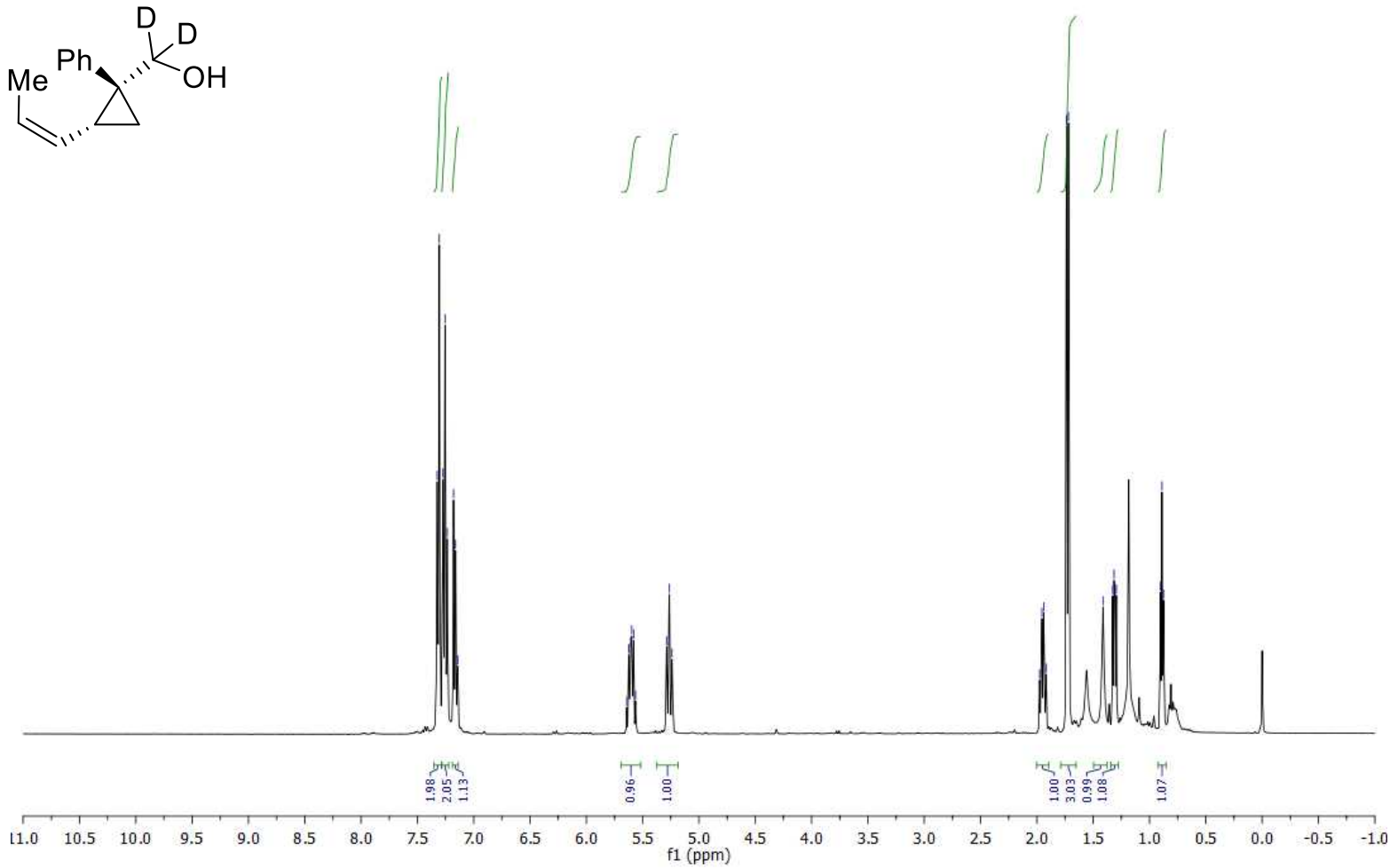

Anthony400-2019

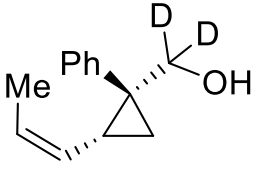

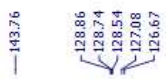

素䇰部素
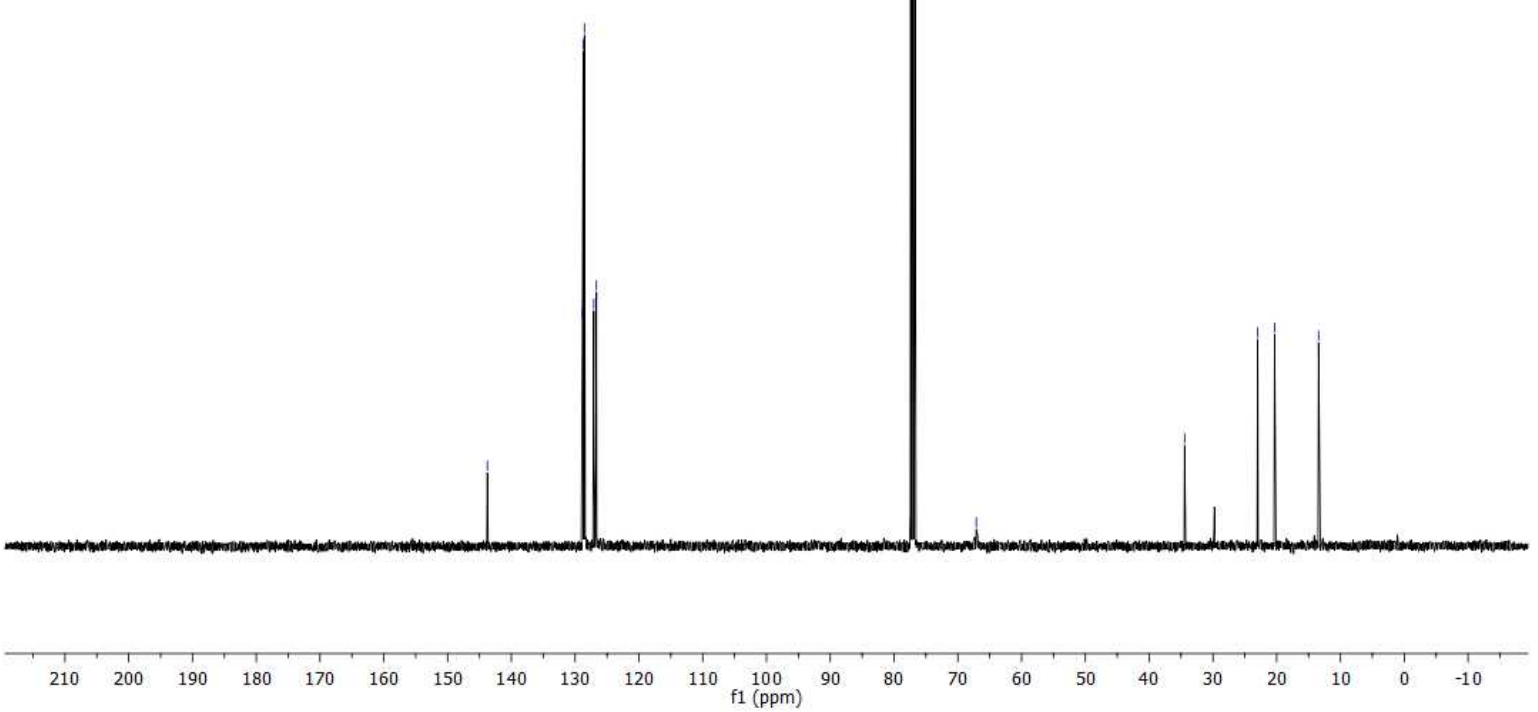

S25 

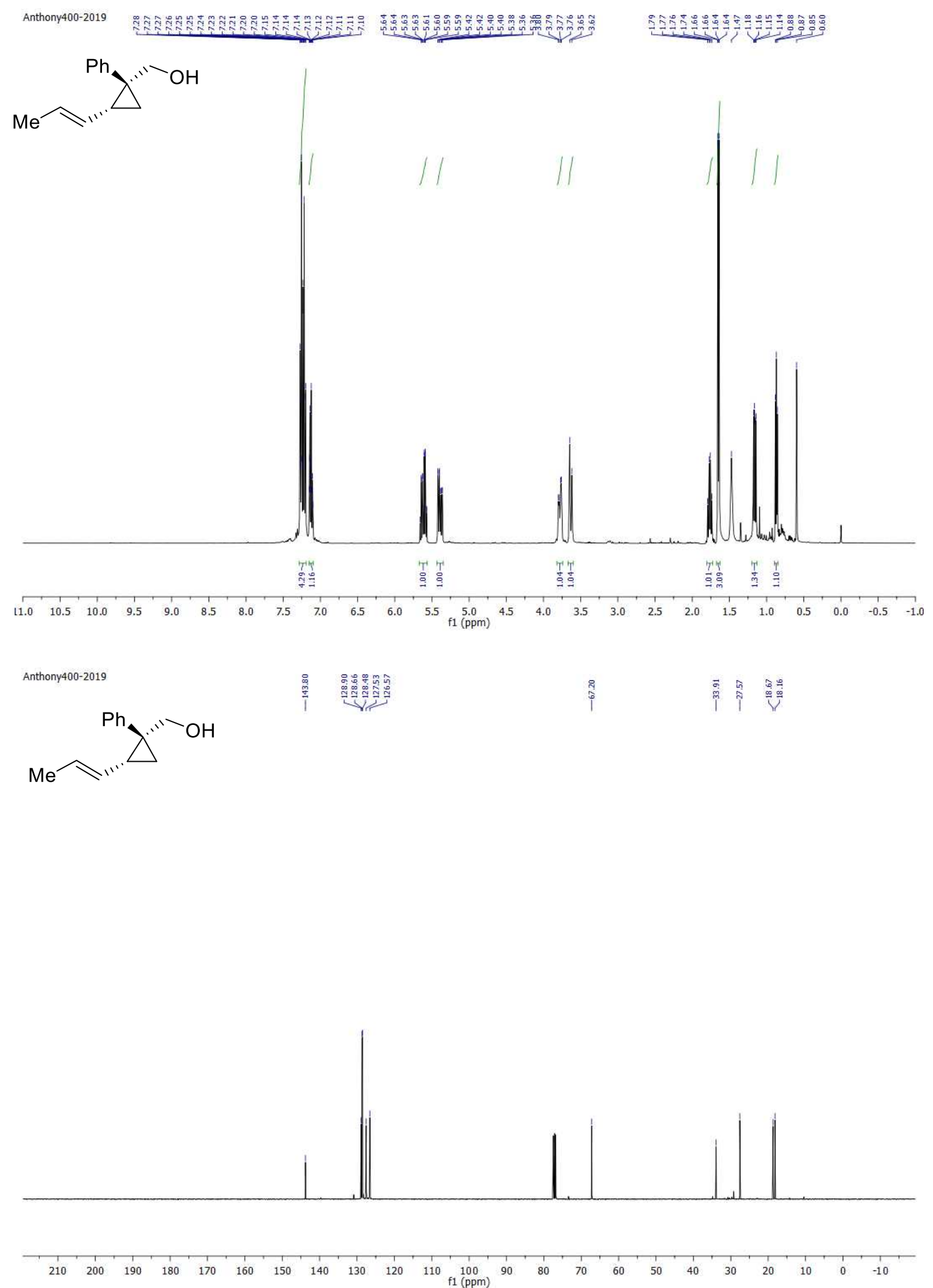


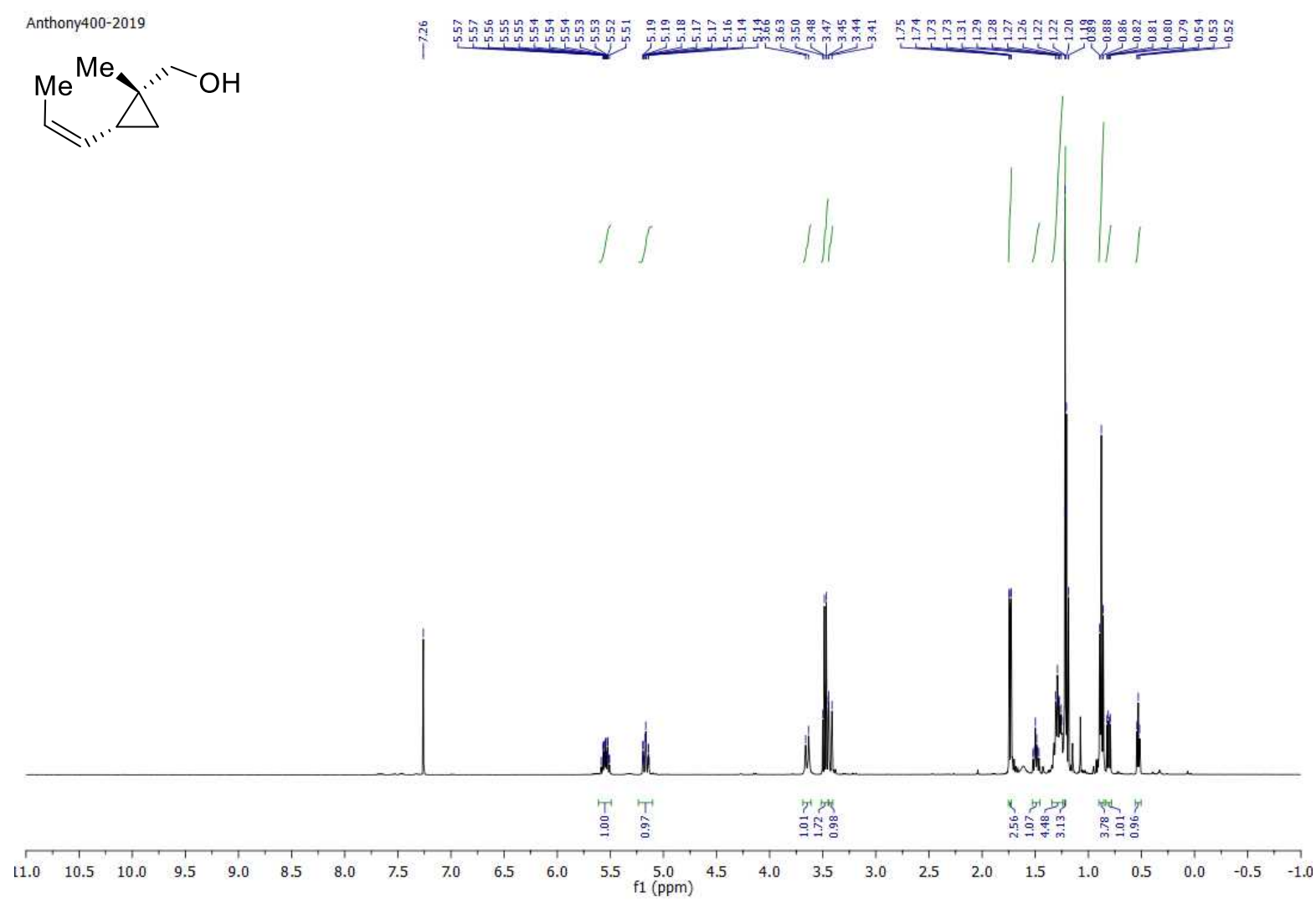

Anthony400-2019

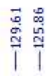

竞:

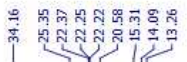

$\begin{array}{llllllllllll}210 & 200 & 190 & 180 & 170 & 160 & 150 & 140 & 130 & 120 & 110 & \begin{array}{l}100 \\ \mathrm{f} 1(\mathrm{ppm})\end{array}\end{array}$ 


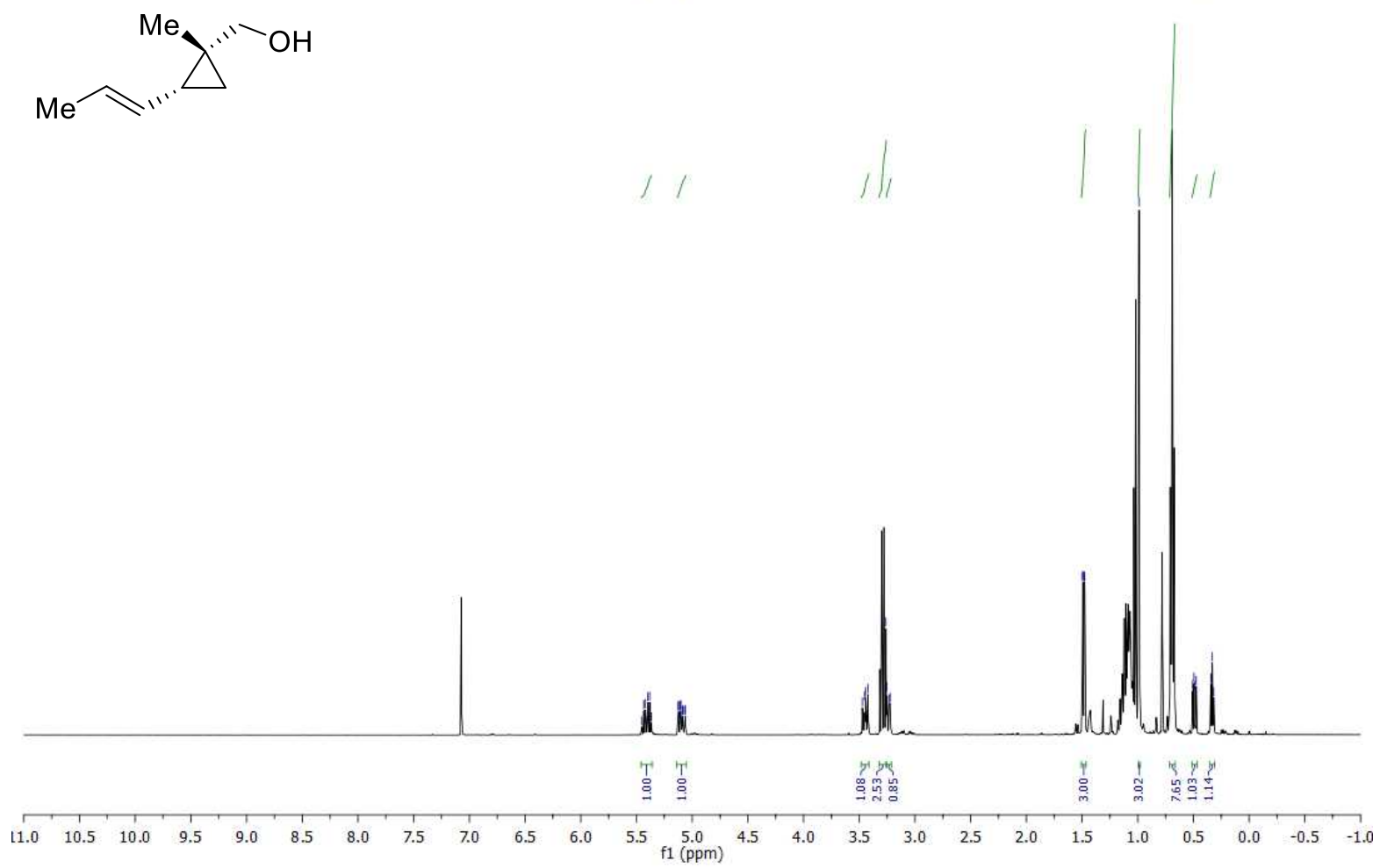

Anthony400-2019<smiles>C/C=C/[C@@H]1C[C@]1(C)CO</smiles> 

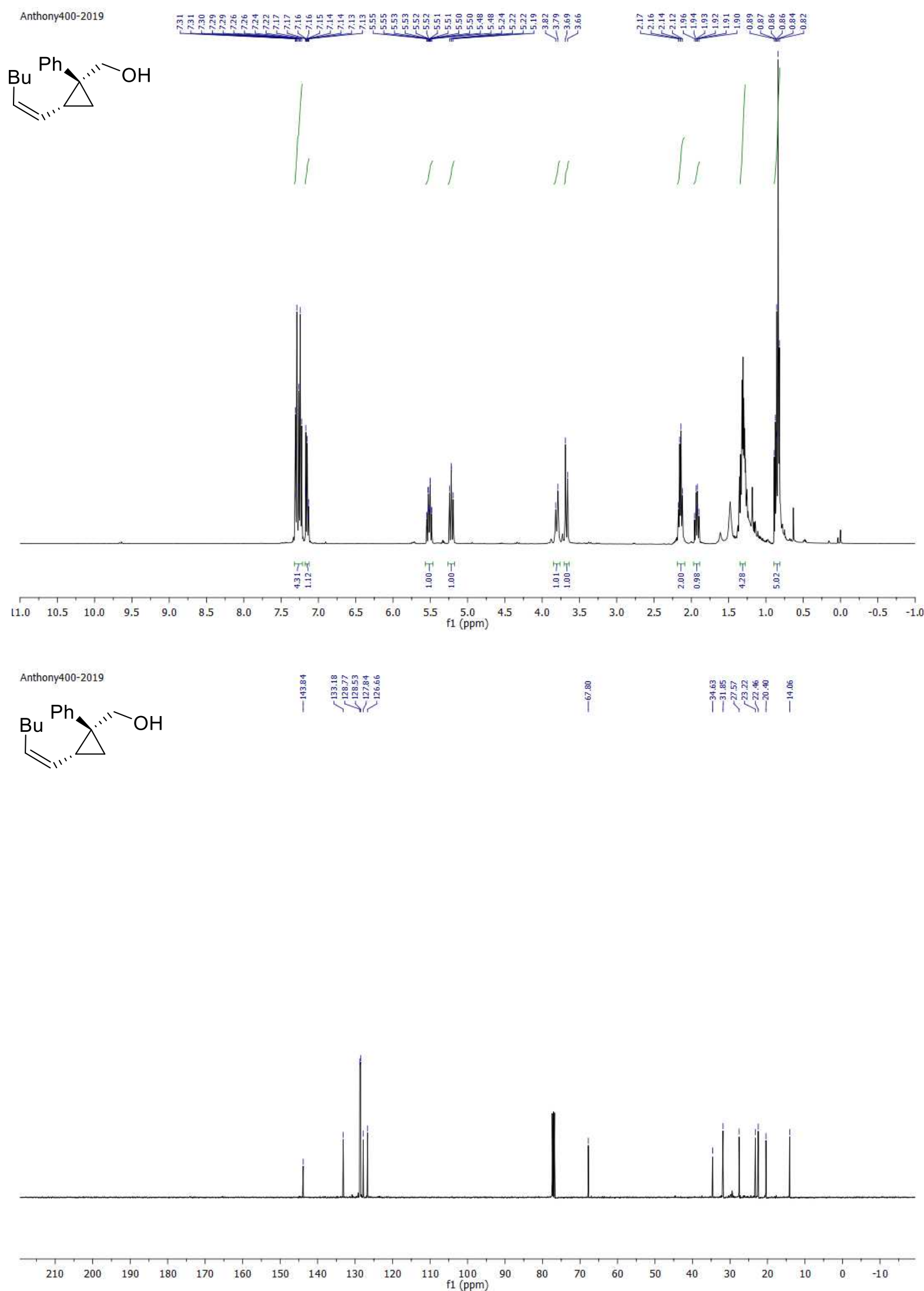

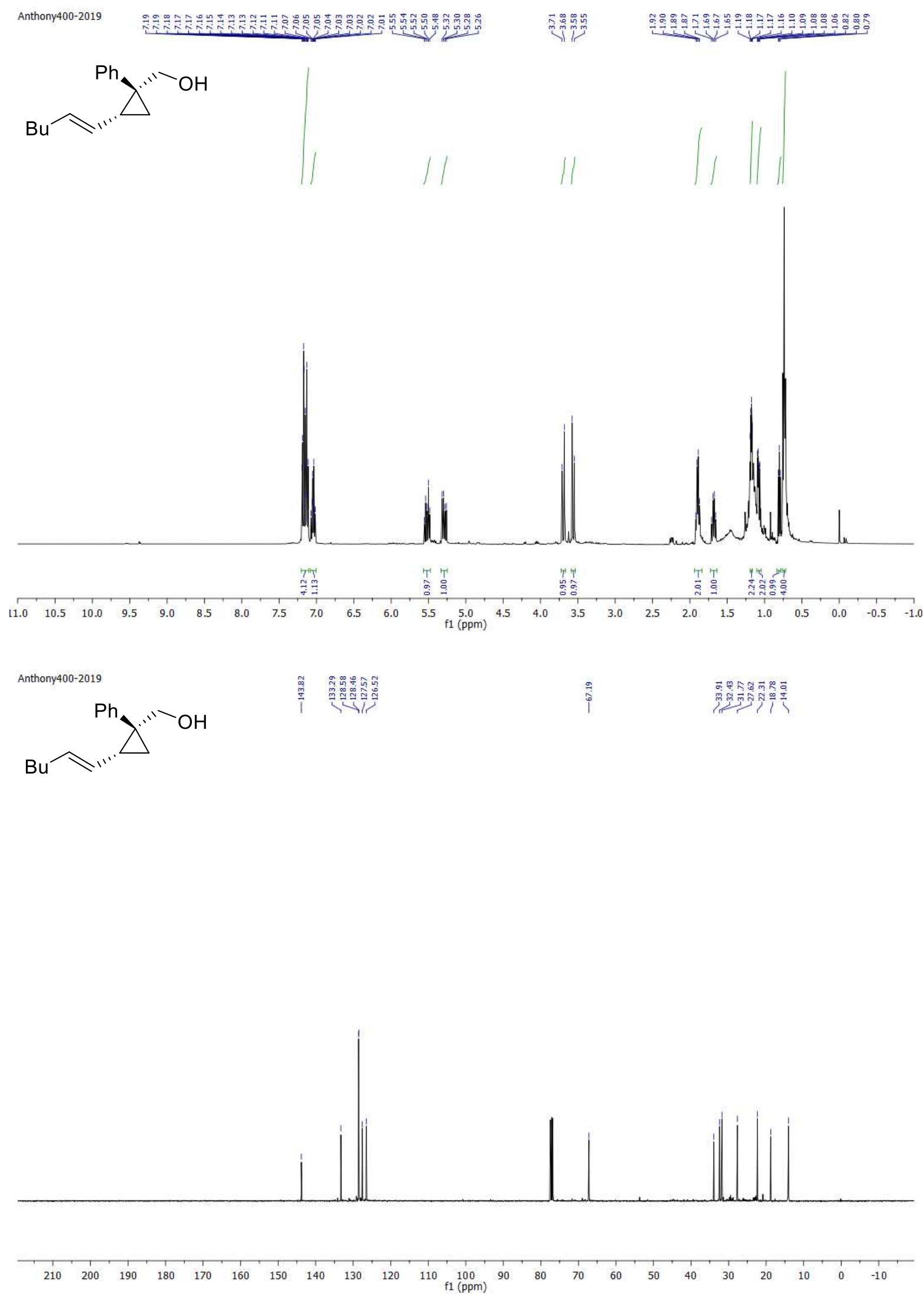
<smiles>CC(C)=C[C@H]1C[C@@]1(CO)c1ccccc1</smiles><smiles>C=C[Hg]</smiles><smiles>c1ccccc1</smiles>

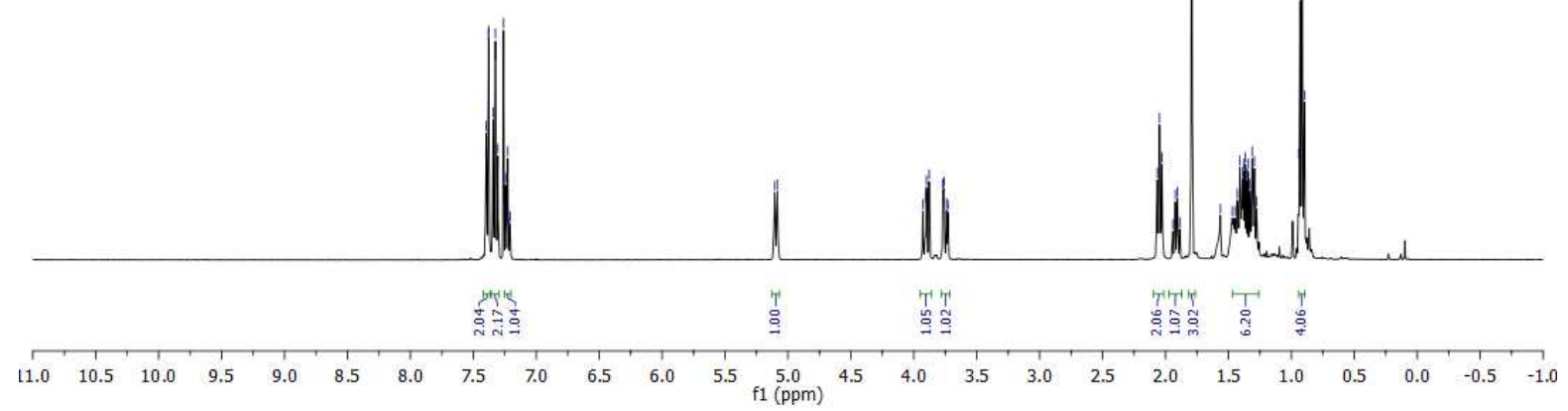

Anthony400-2019<smiles>CC(C)=C[C@H]1C[C@]1(CO)c1ccccc1</smiles> 


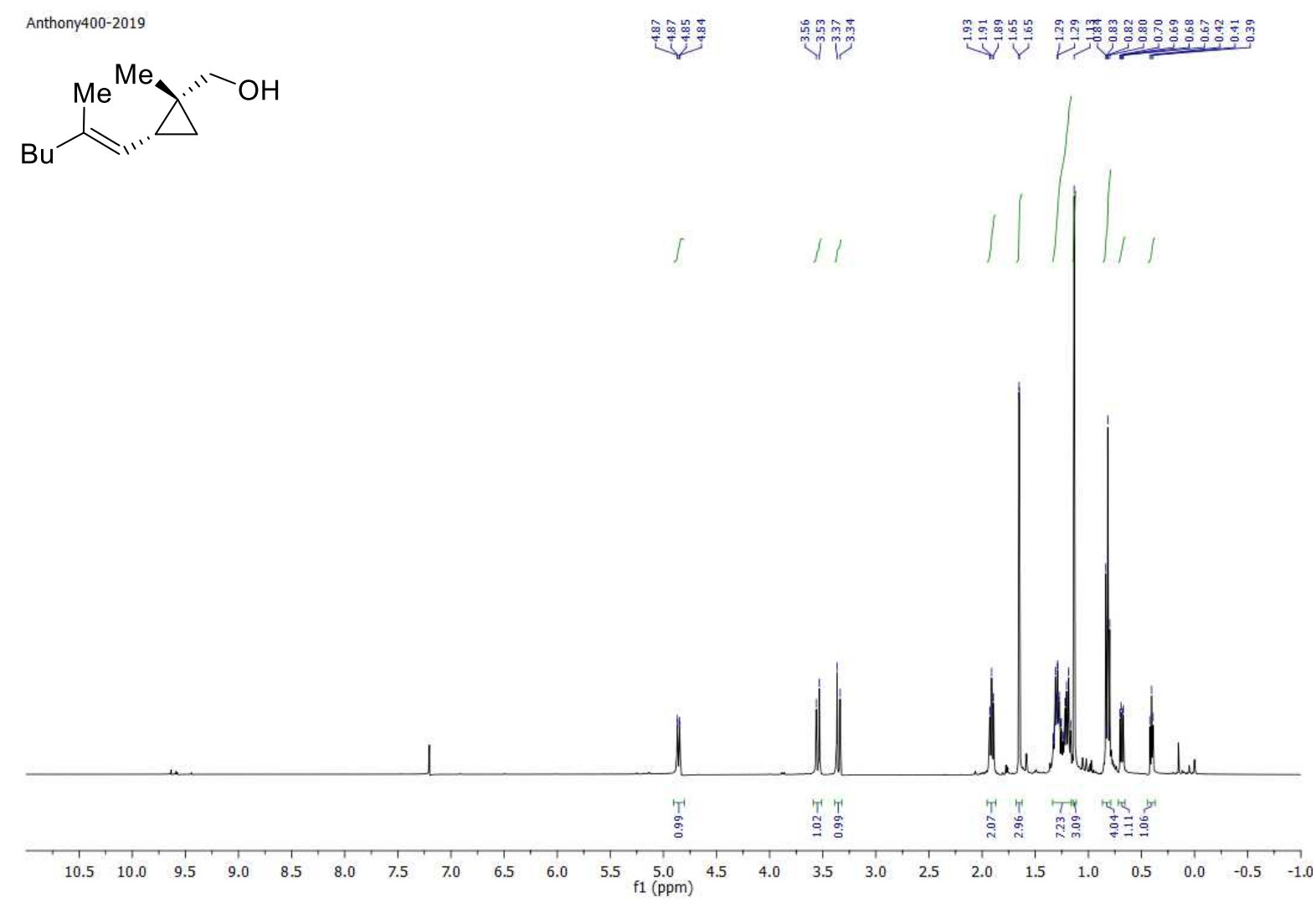

Anthony400-2019<smiles>CC(=C[C@@H]1C[C@@]1(C)CO)CBr</smiles>
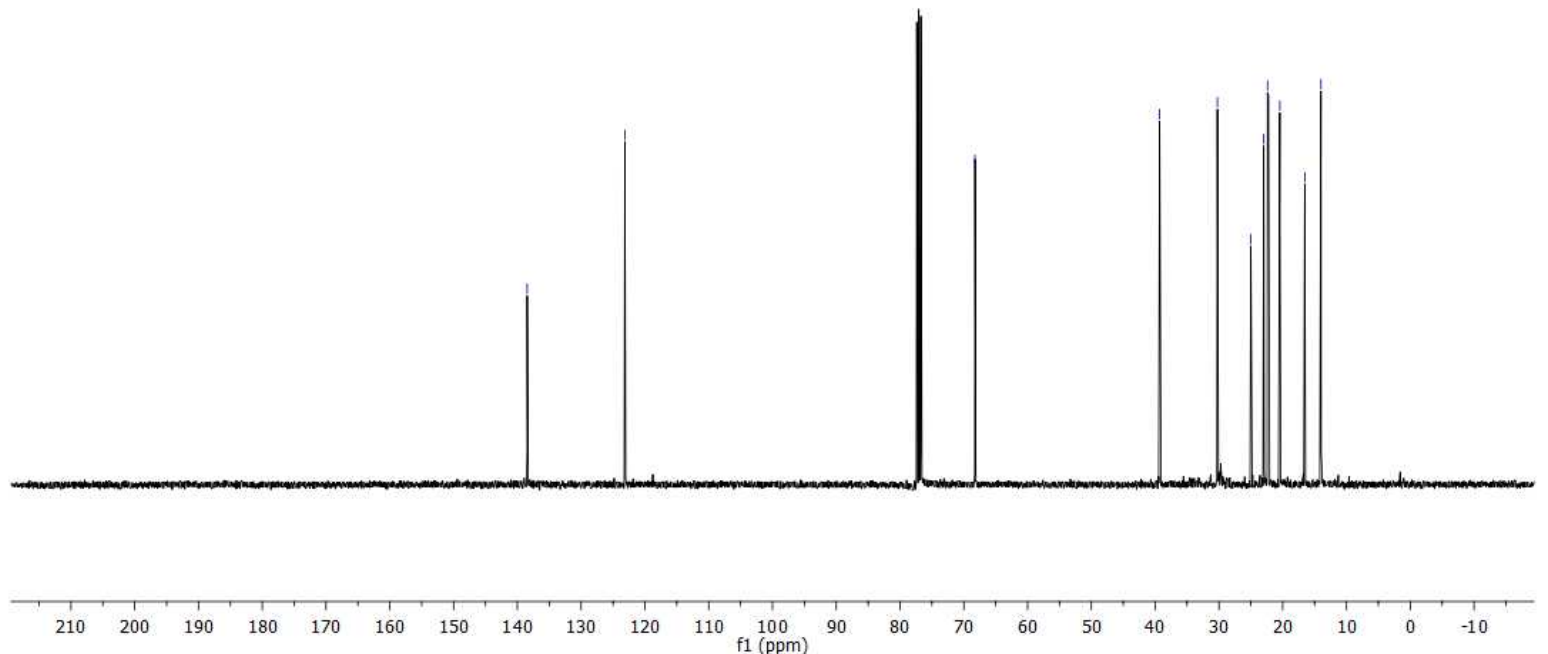

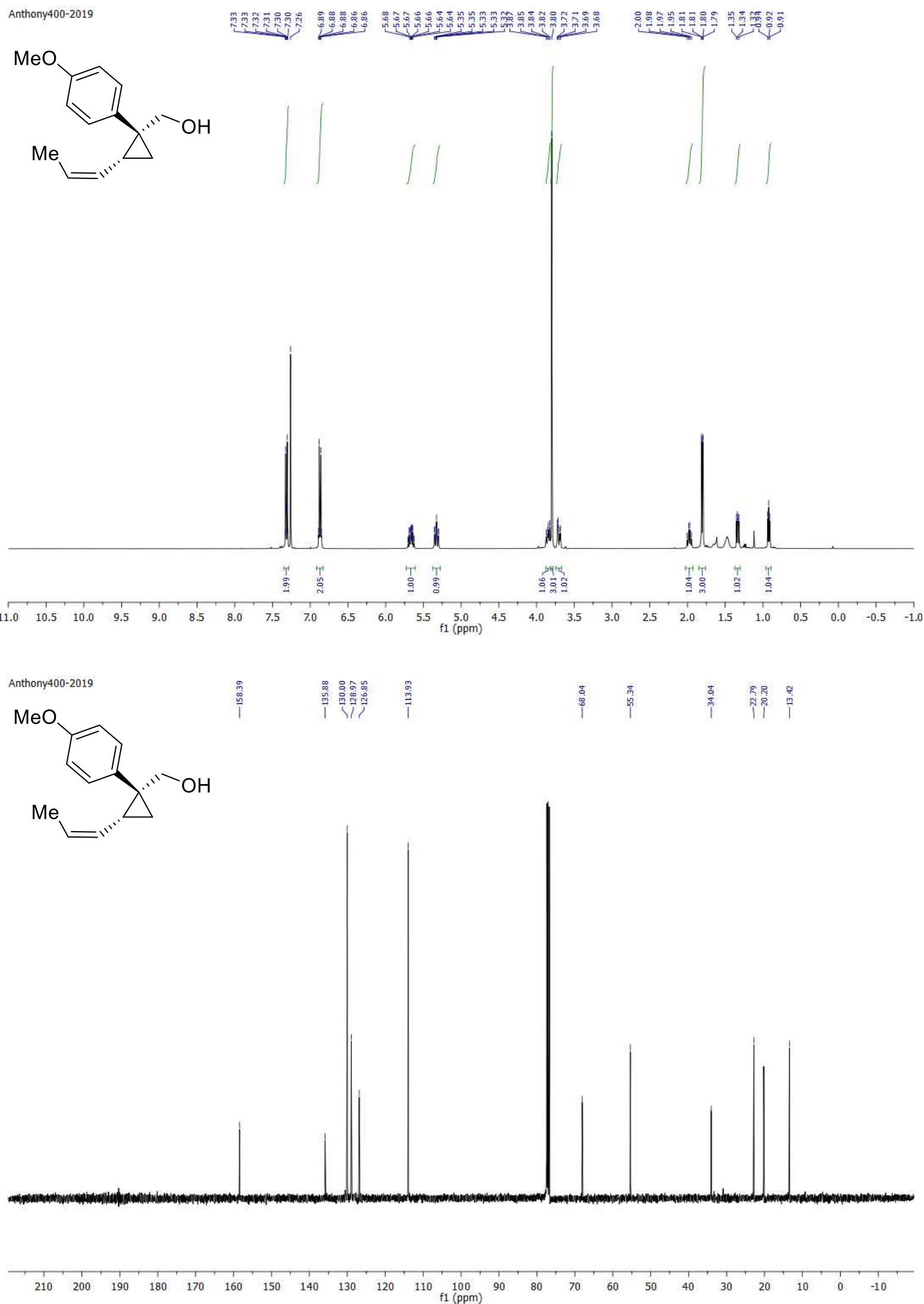

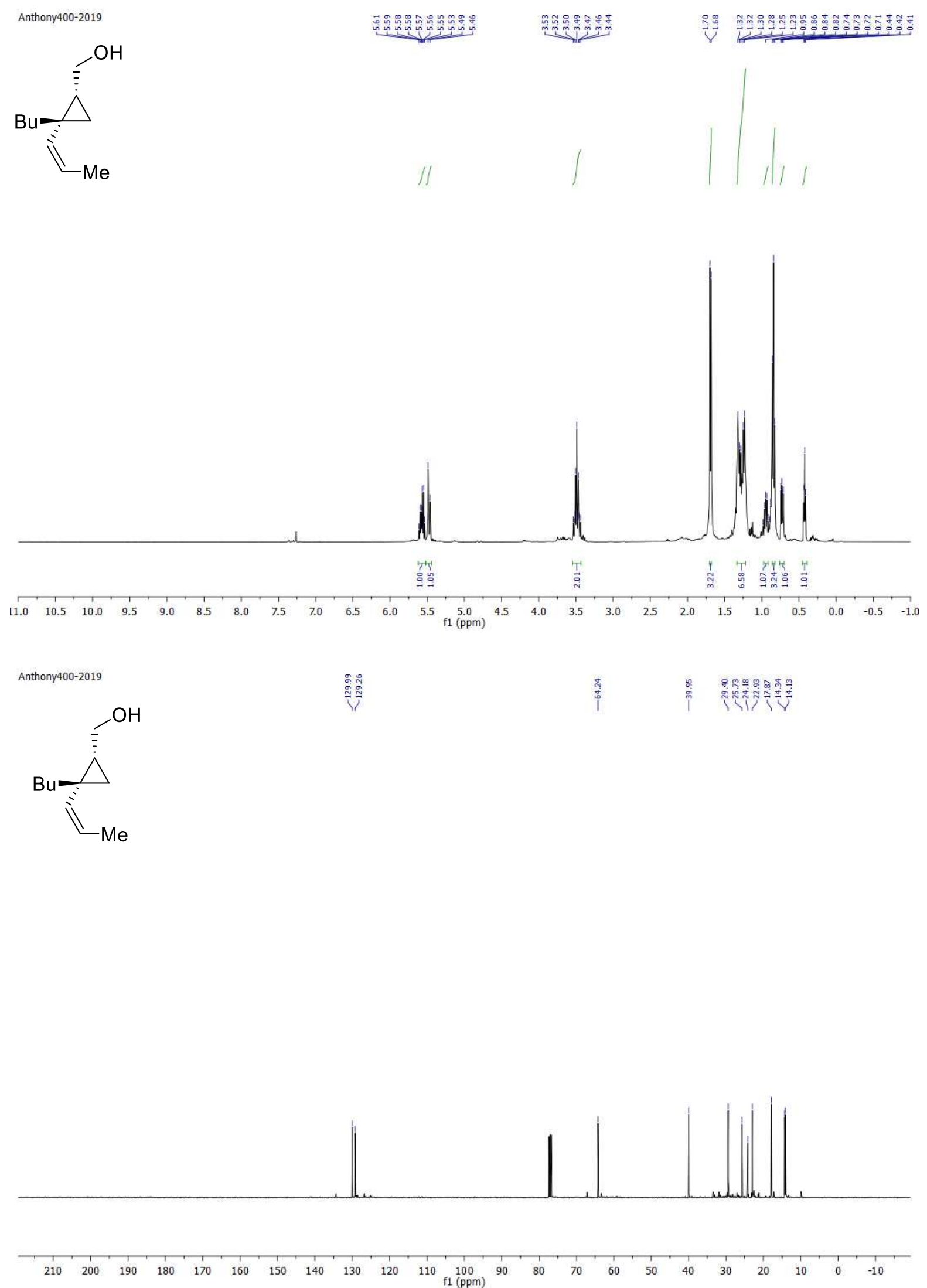
<smiles>Cc1ccc([C@H](C)/C=C/CC(CO)c2ccccc2)cc1</smiles>

\section{\|\|}
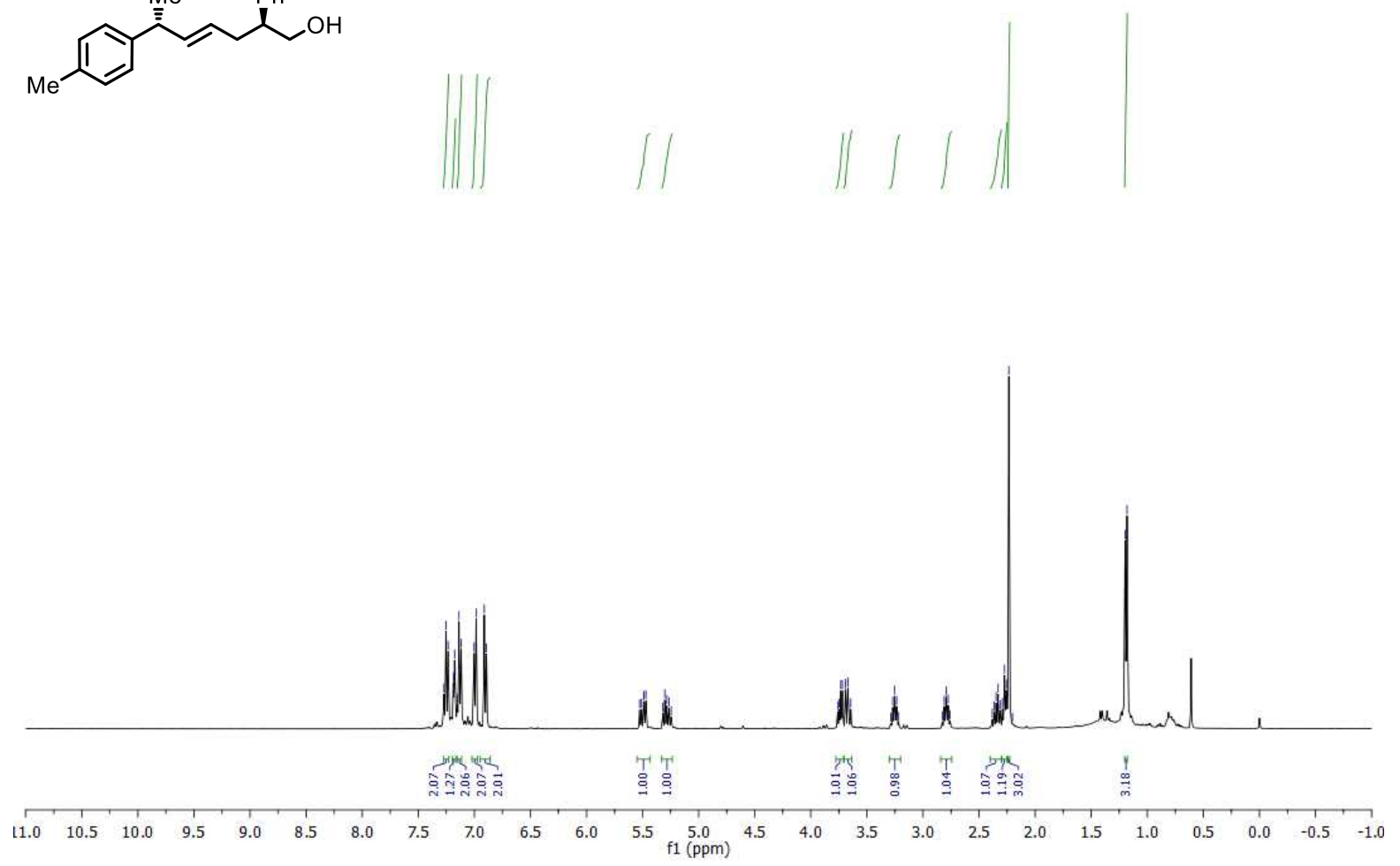

Anthony400-2019

$\overbrace{}^{M e}$

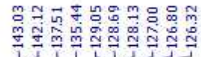

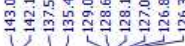

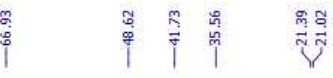
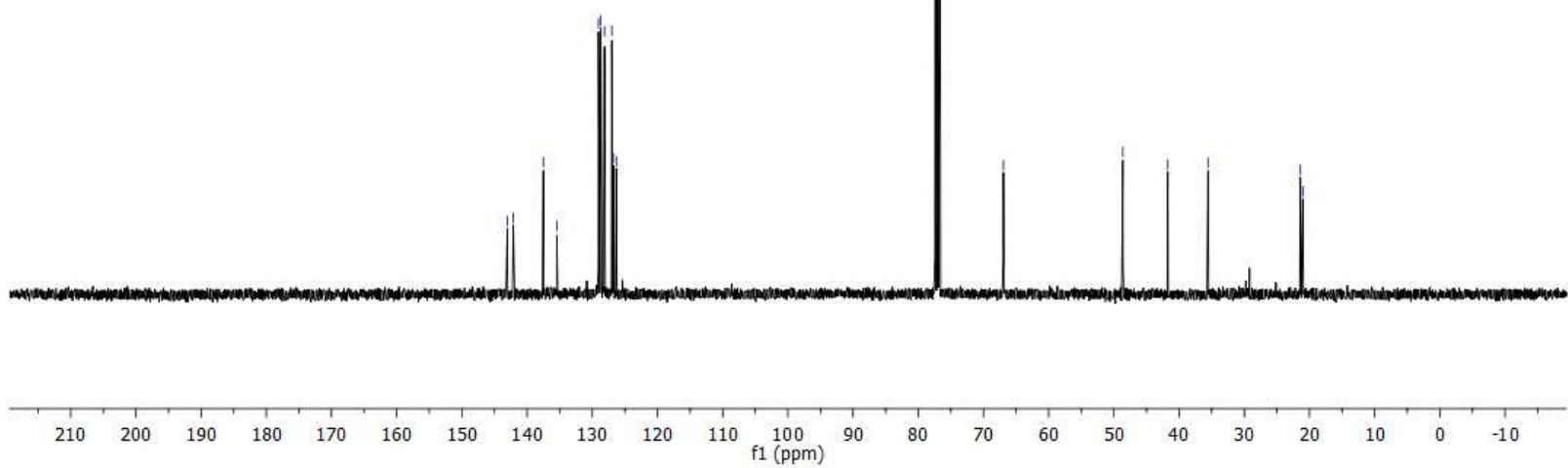
<smiles>COc1ccc([C@H](C)/C=C/CC(c2ccccc2)c2ccccc2)cc1</smiles>
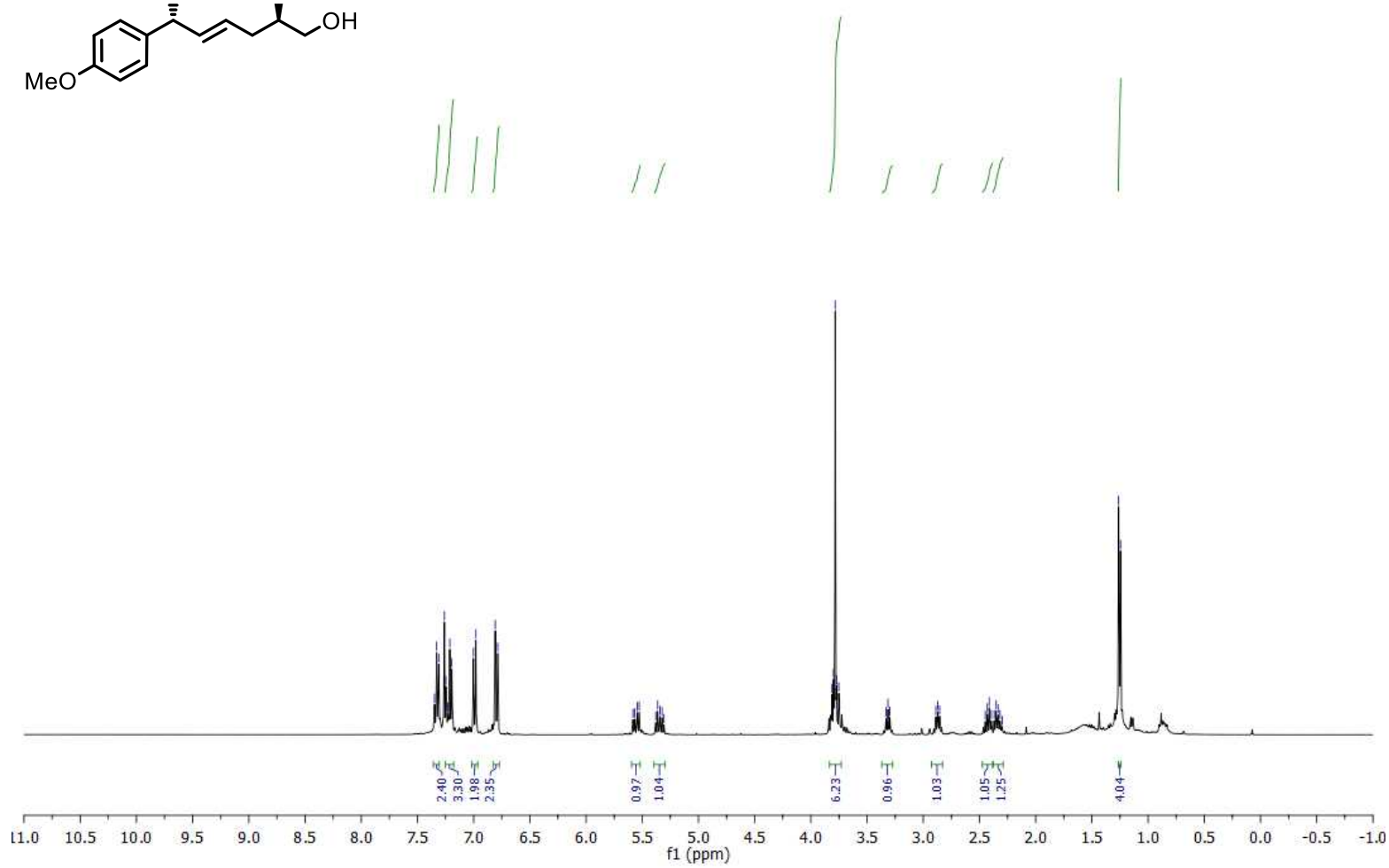

Anthony400-2019

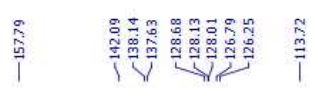

ind<smiles>COc1ccc([C@H](C)/C=C/CC(c2ccccc2)c2ccccc2)cc1</smiles>

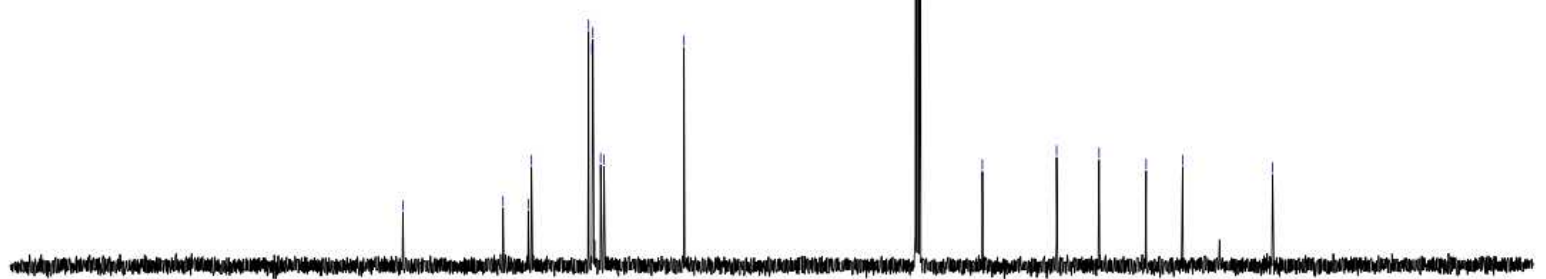

$\begin{array}{lllllllllllllllllllllll}210 & 200 & 190 & 180 & 170 & 160 & 150 & 140 & 130 & 120 & 110 & \begin{array}{l}100 \\ \mathrm{f} 1(\mathrm{ppm})\end{array} & 90 & 80 & 70 & 60 & 50 & 40 & 30 & 20 & 10 & 0 & -10\end{array}$ 


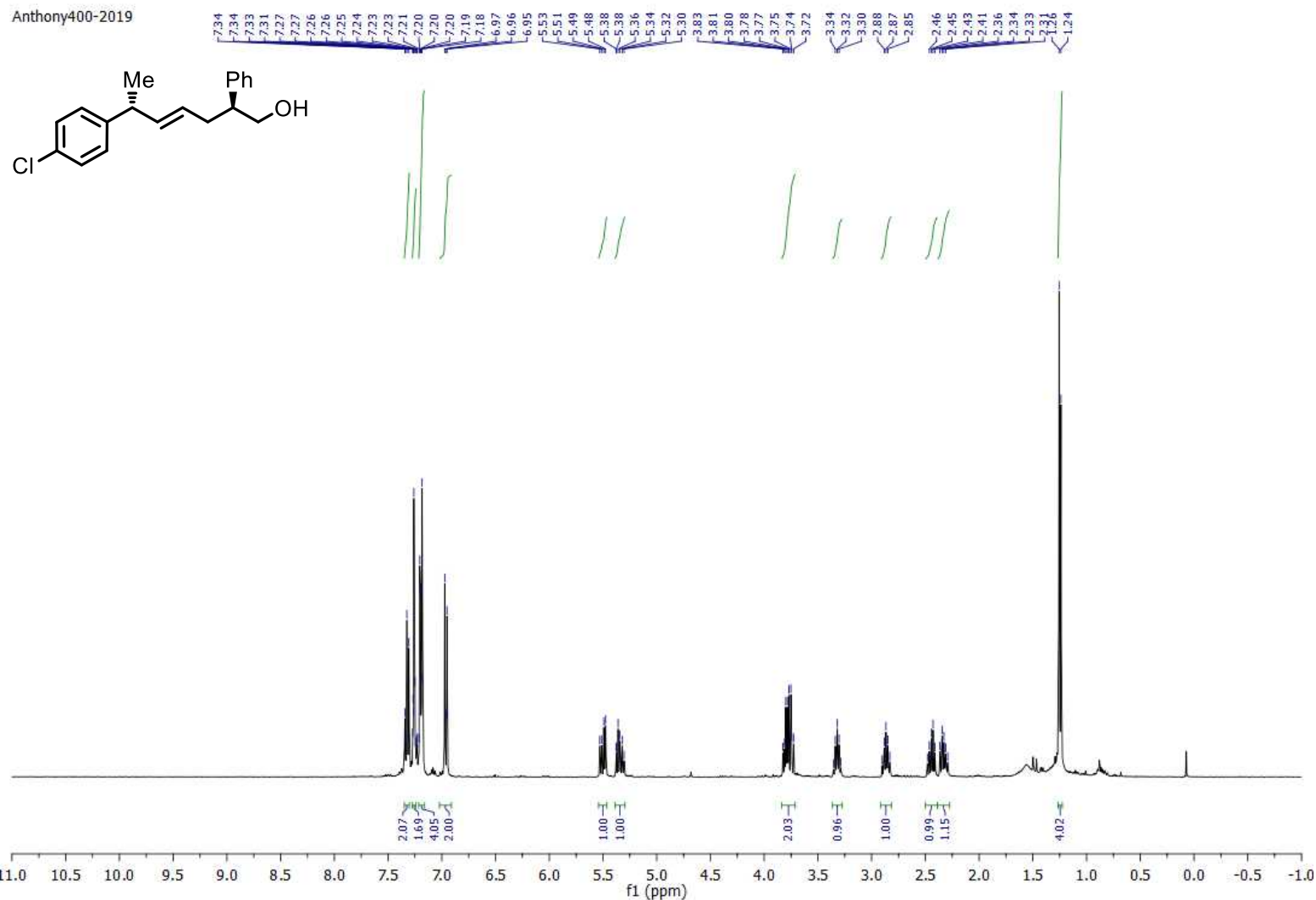

Anthony400-2019<smiles>C[C@H](C=CCC(O)c1ccccc1)c1ccc(Cl)cc1</smiles>

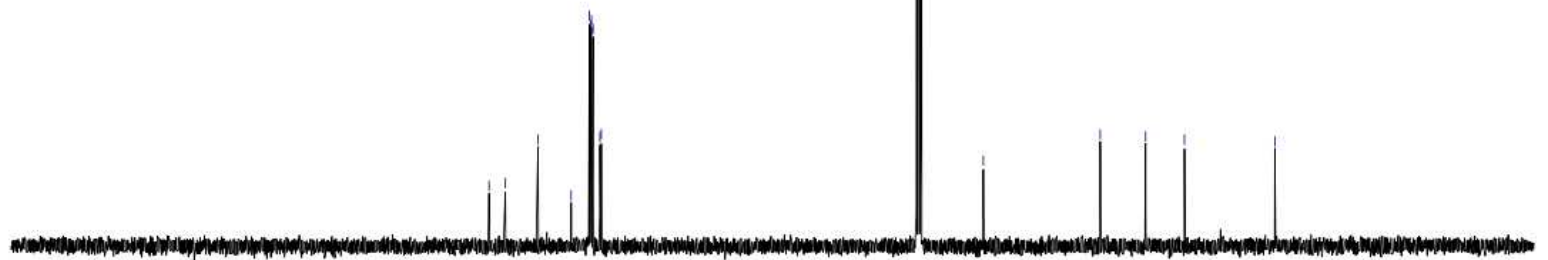

$\begin{array}{lllllllllllllllllllllll}210 & 200 & 190 & 180 & 170 & 160 & 150 & 140 & 130 & 120 & 110 & \begin{array}{l}100 \\ \mathrm{f} 1(\mathrm{ppm})\end{array} & 90 & 80 & 70 & 60 & 50 & 40 & 30 & 20 & 10 & 0 & -10\end{array}$ 
Anthony400-2019

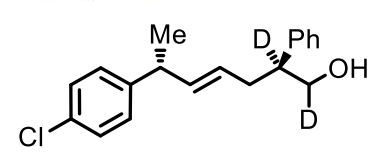
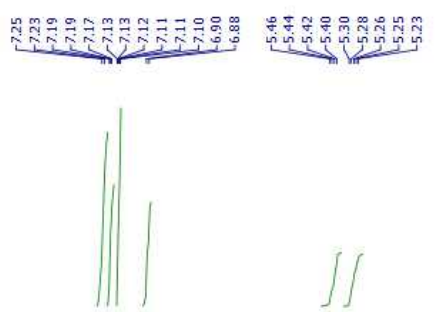

$\iint$

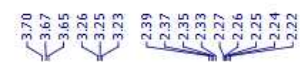

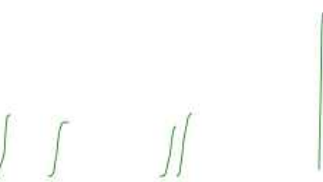

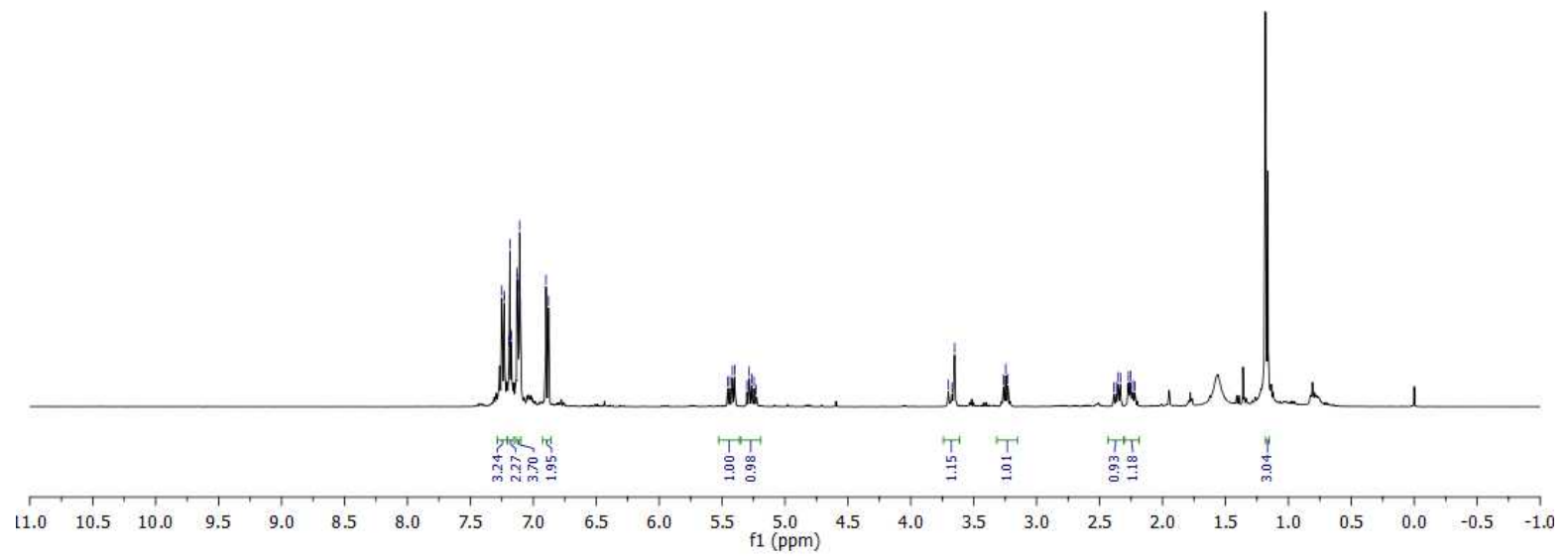

Anthony400-2019<smiles>[2H]C(O)[C@@]([2H])(c1ccccc1)[P+]([2H])([2H])C/C=C/[C@H](C)c1ccc(Cl)cc1</smiles>

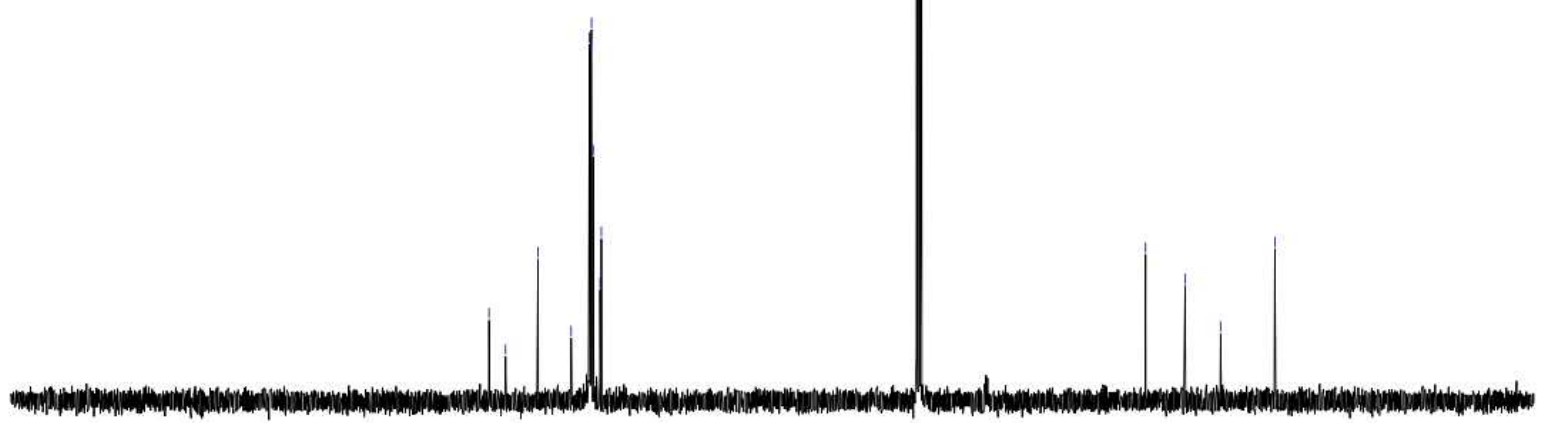

$\begin{array}{llllllllllllllllllllllll}210 & 200 & 190 & 180 & 170 & 160 & 150 & 140 & 130 & 120 & 110 & \begin{array}{l}100 \\ \mathrm{f} 1(\mathrm{ppm})\end{array} & 90 & 80 & 70 & 60 & 50 & 40 & 30 & 20 & 10 & 0 & -10\end{array}$ 


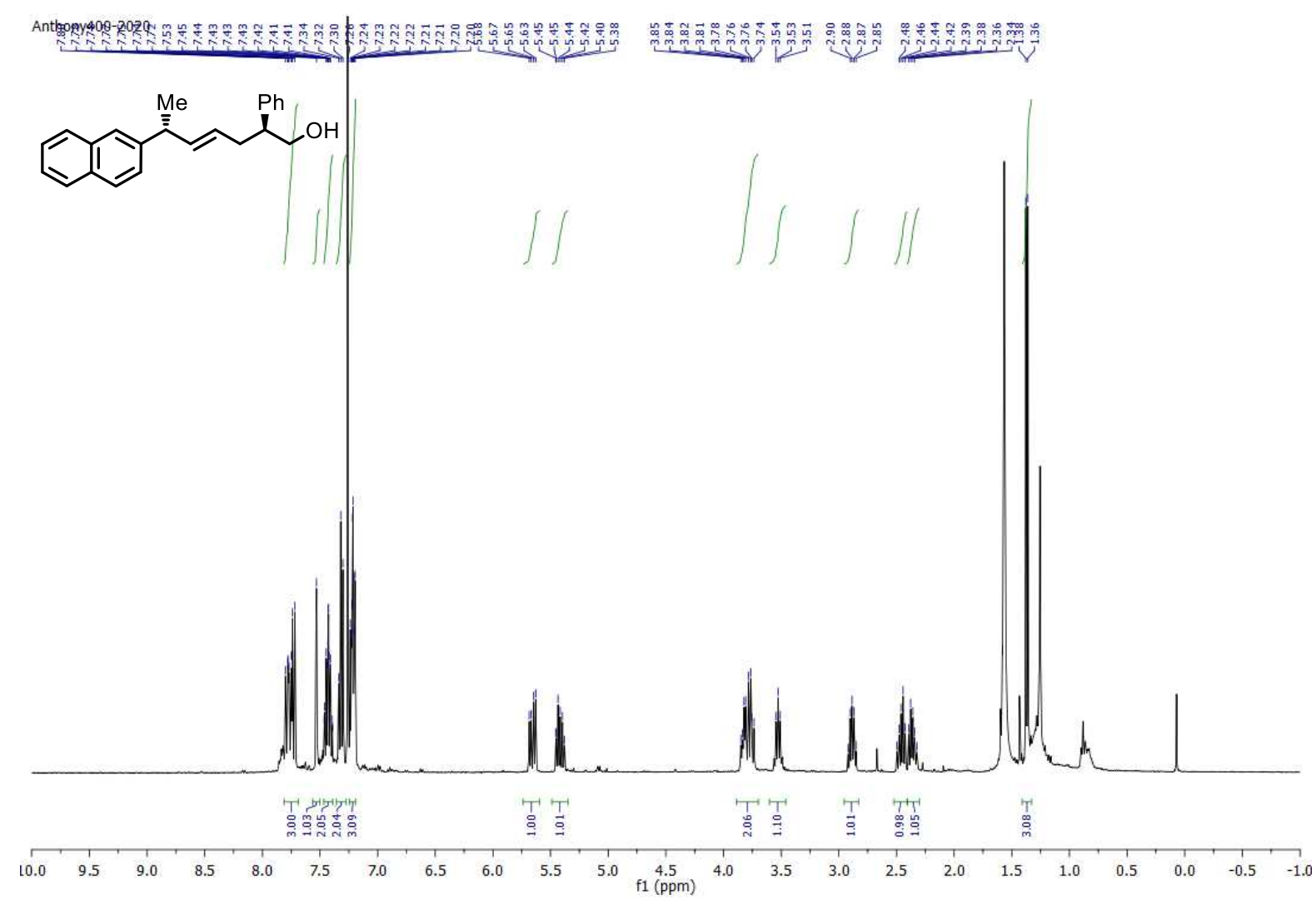

Anthony400-2019

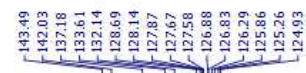

(1)
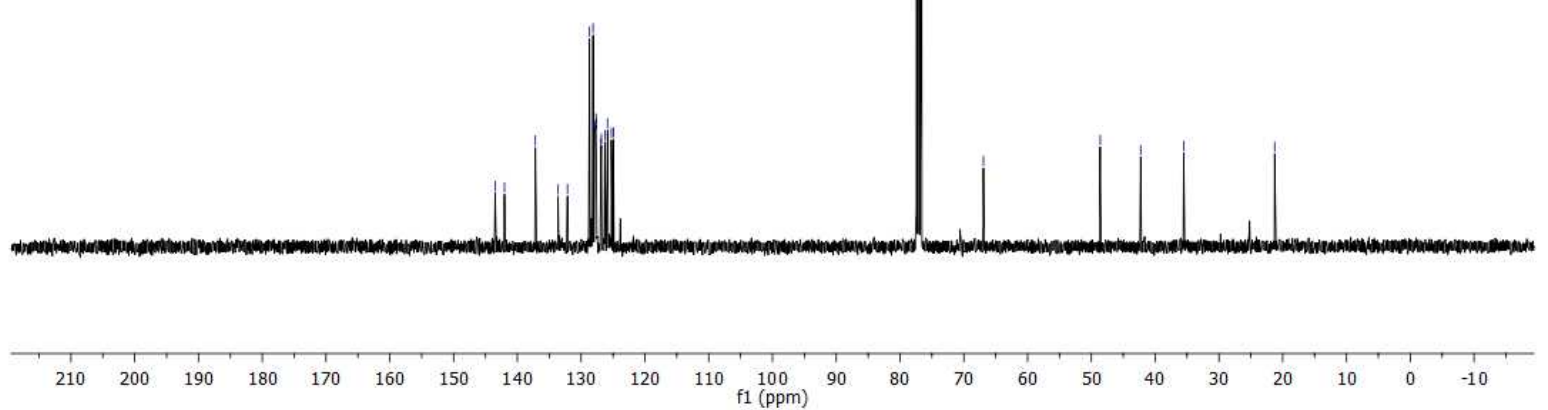

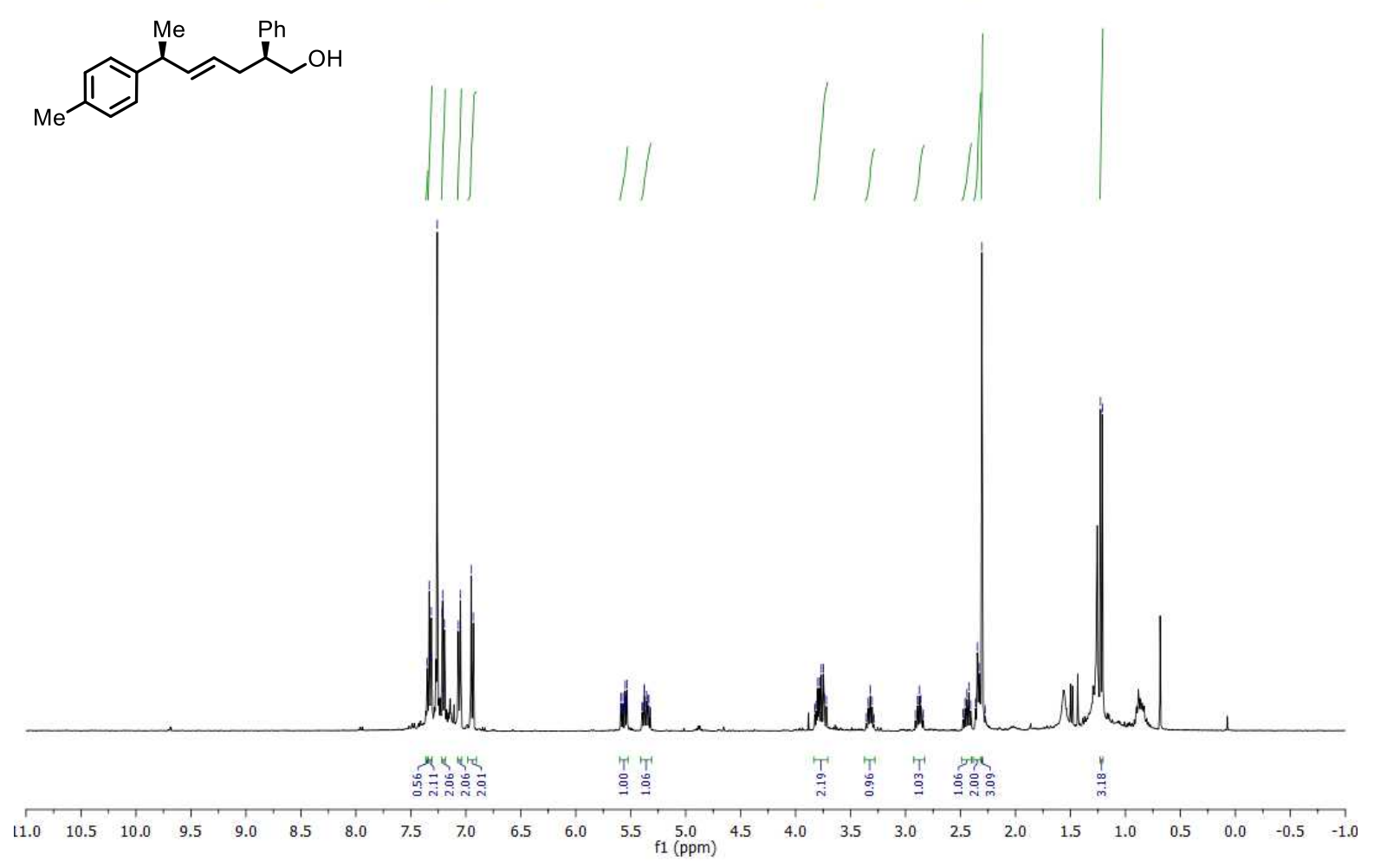

Anthony400-2019

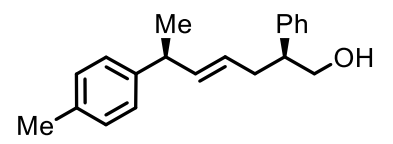

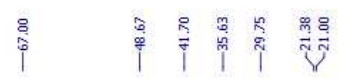
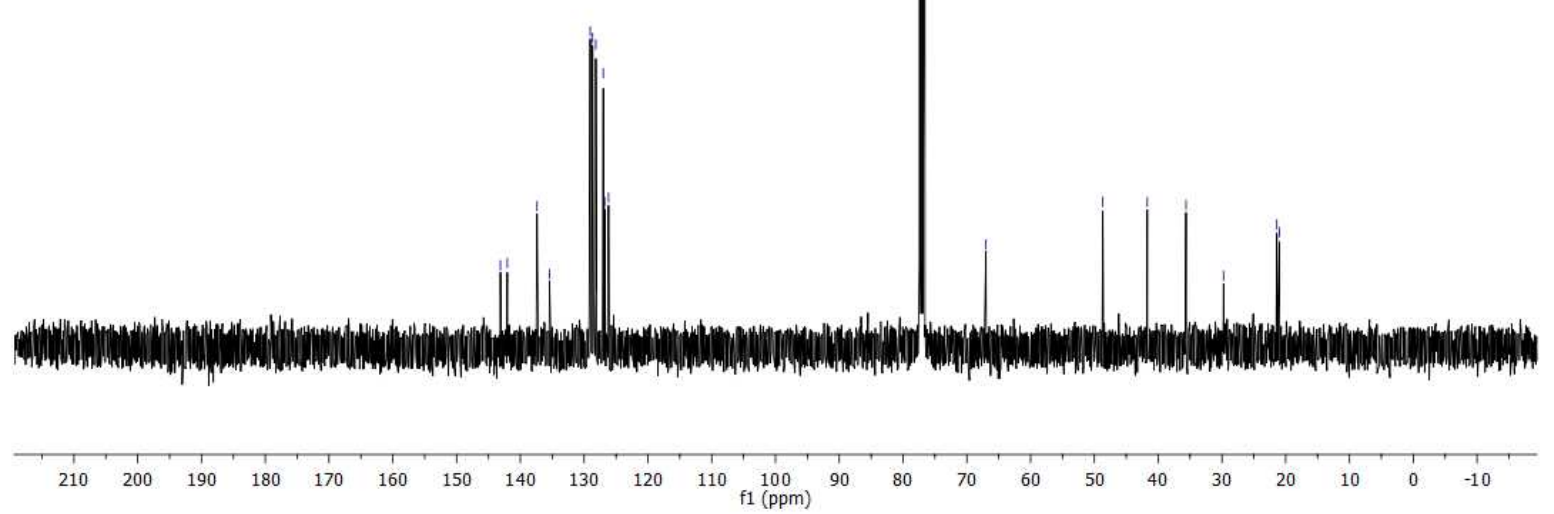


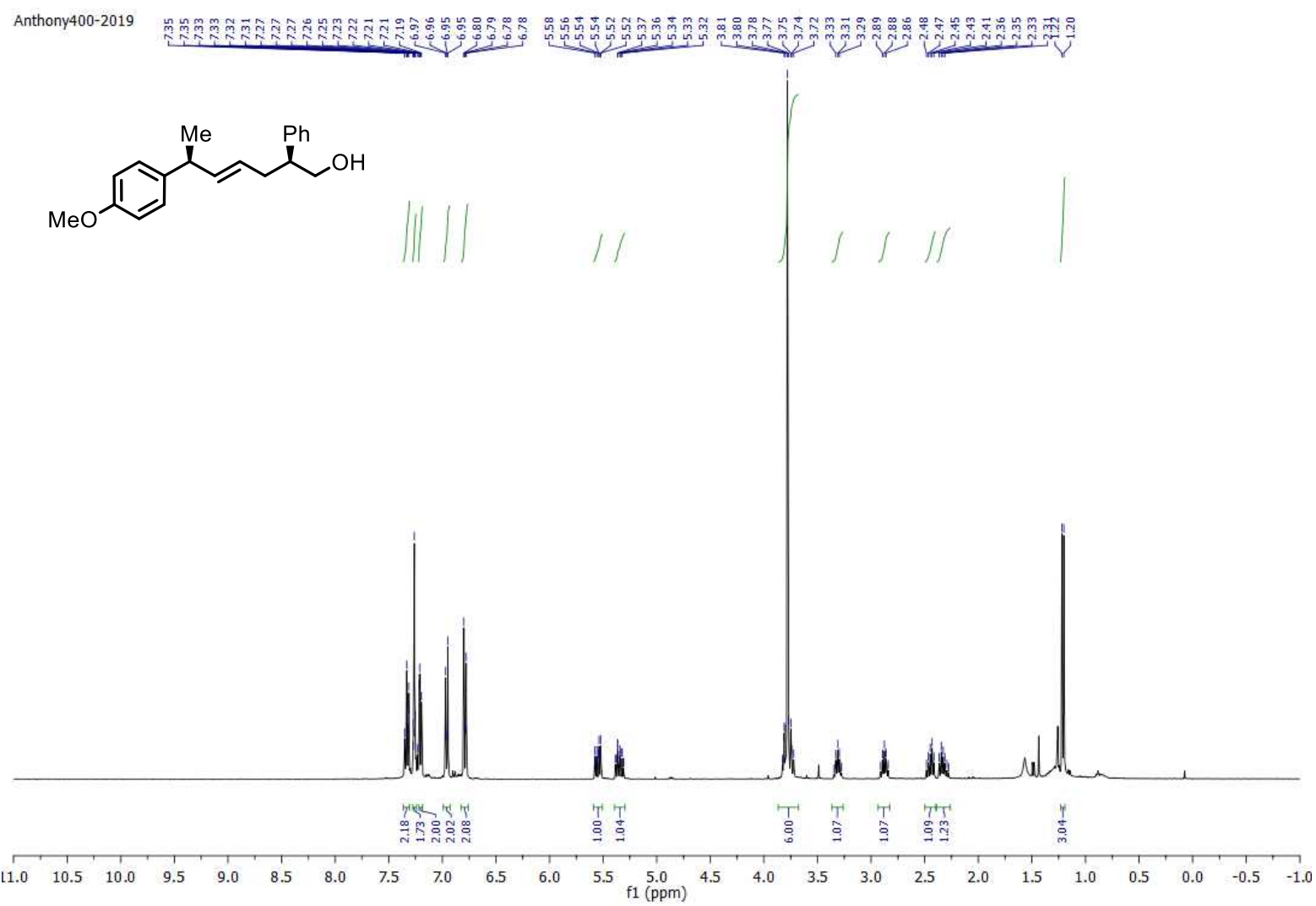

Anthony400-2019

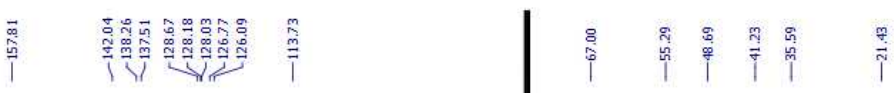<smiles>COc1ccc(C(C)(C)C=CCC(O)c2ccccc2)cc1</smiles>
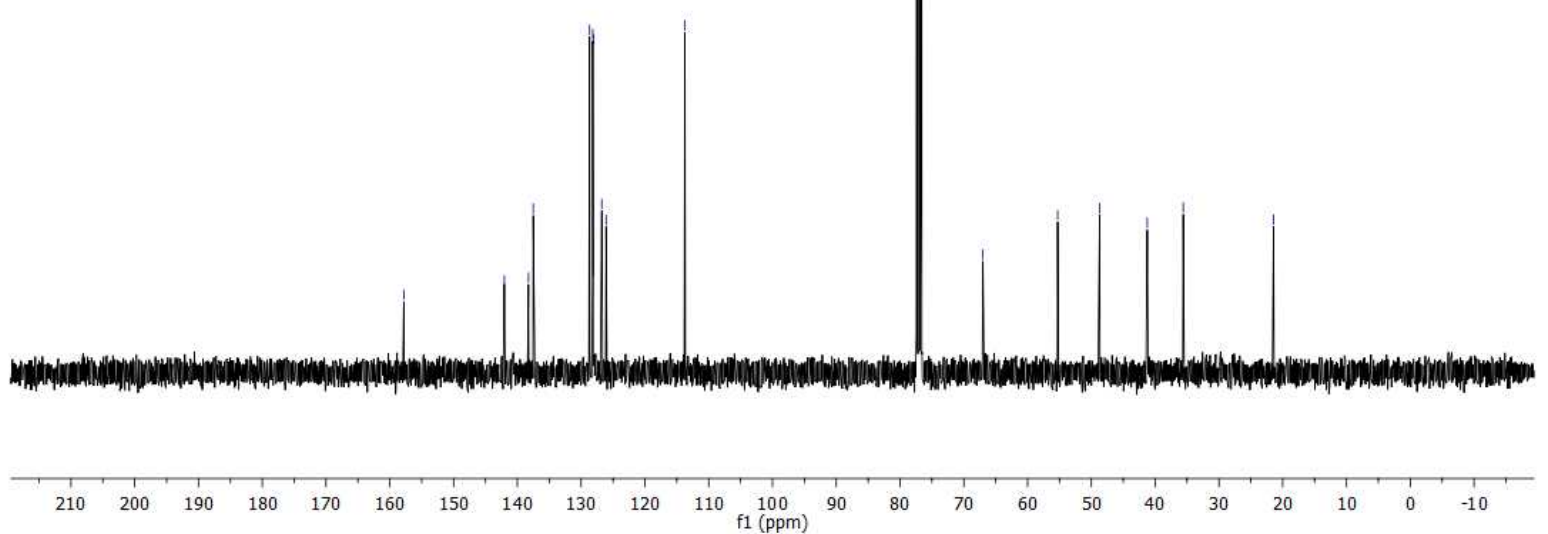
Anthony40-2019<smiles>CC(/C=C/CC(CO)c1ccccc1)c1ccc(Cl)cc1</smiles>

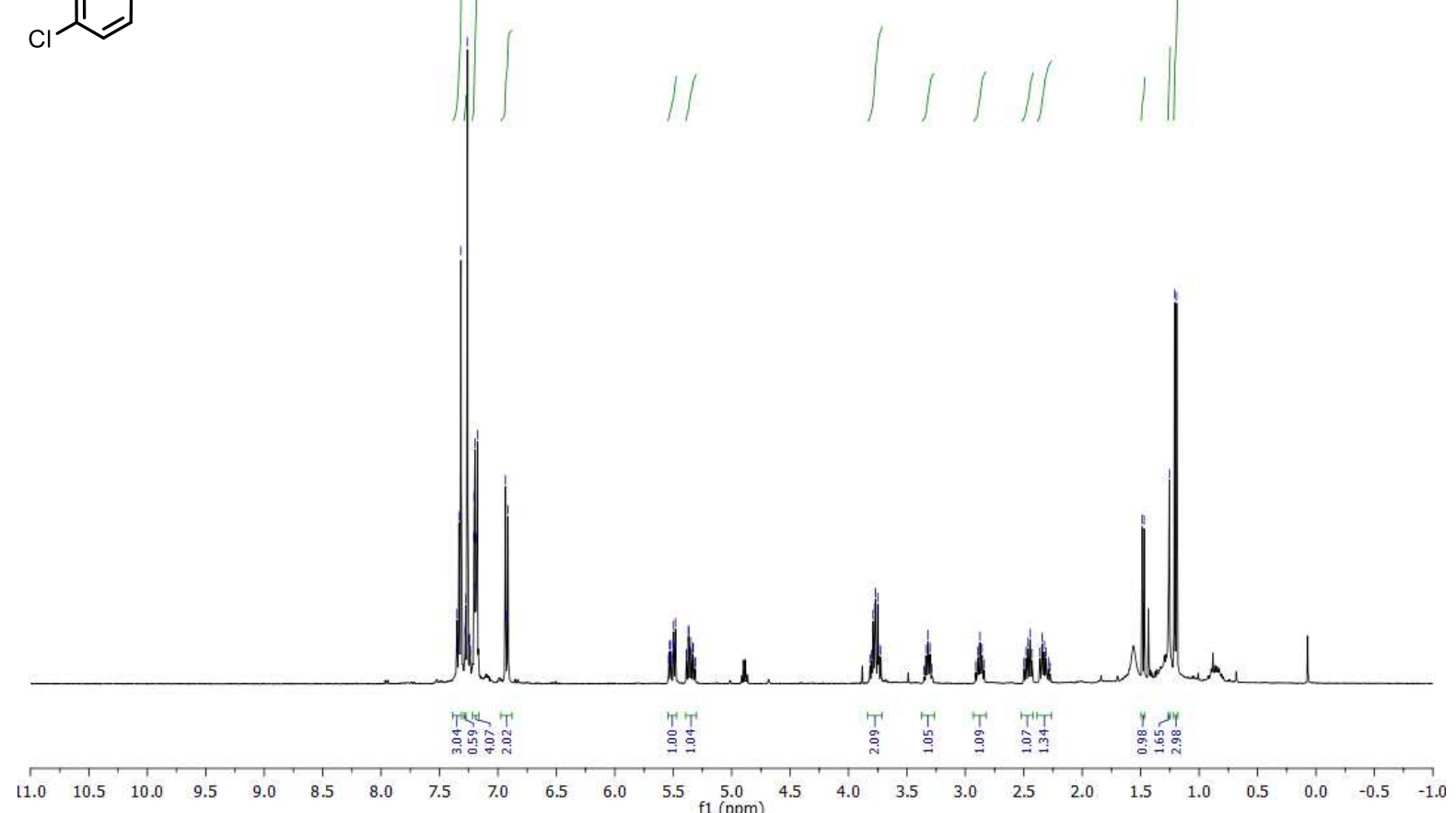<smiles>C[C@H](/C=C/C[C@H](CO)c1ccccc1)c1ccc(Cl)cc1</smiles>

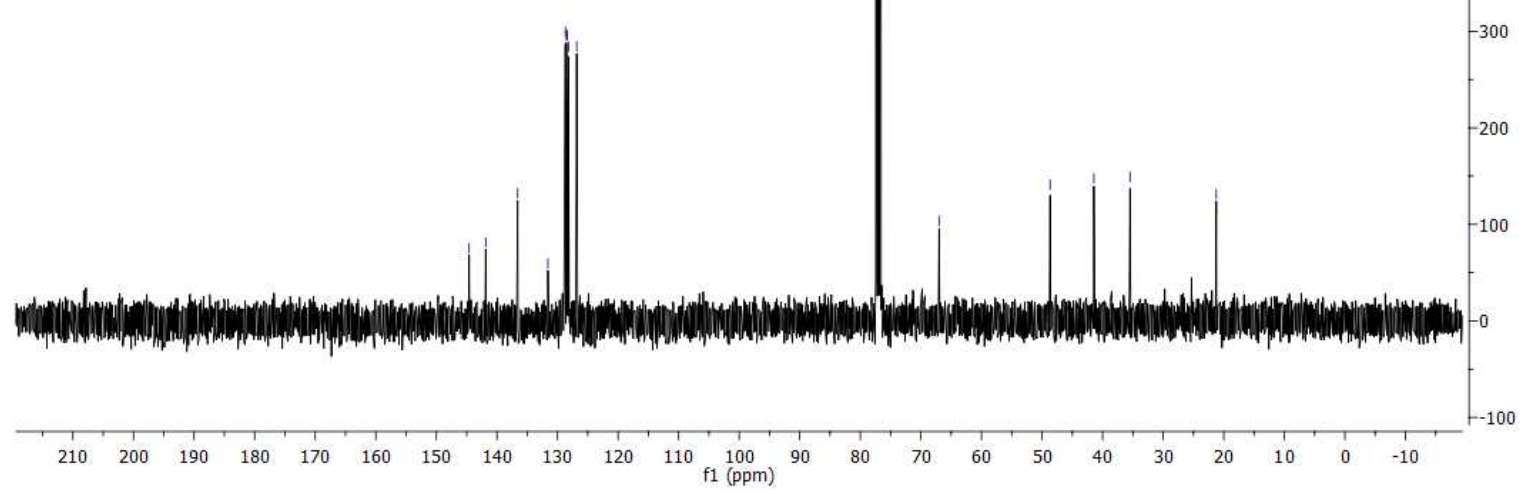



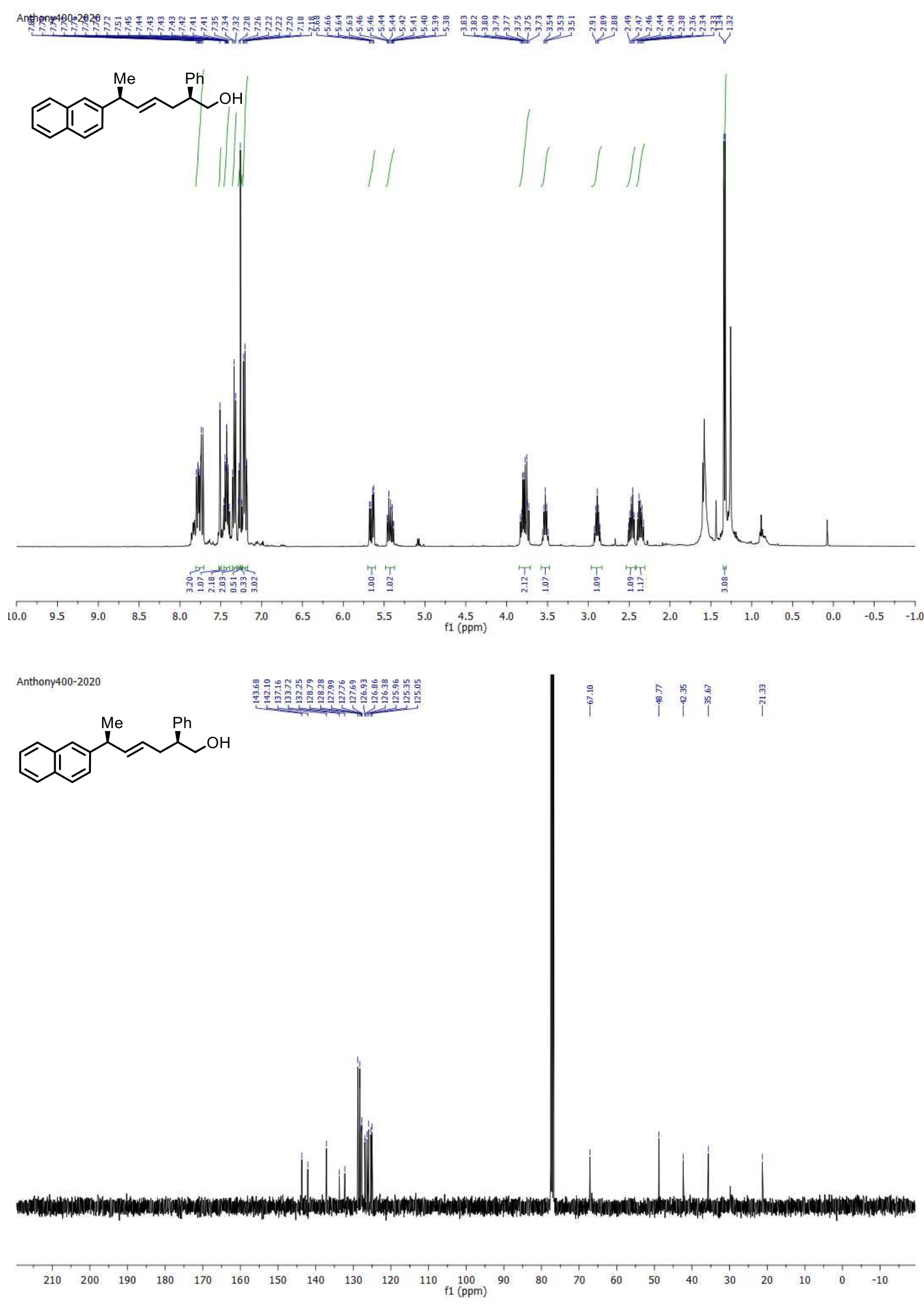

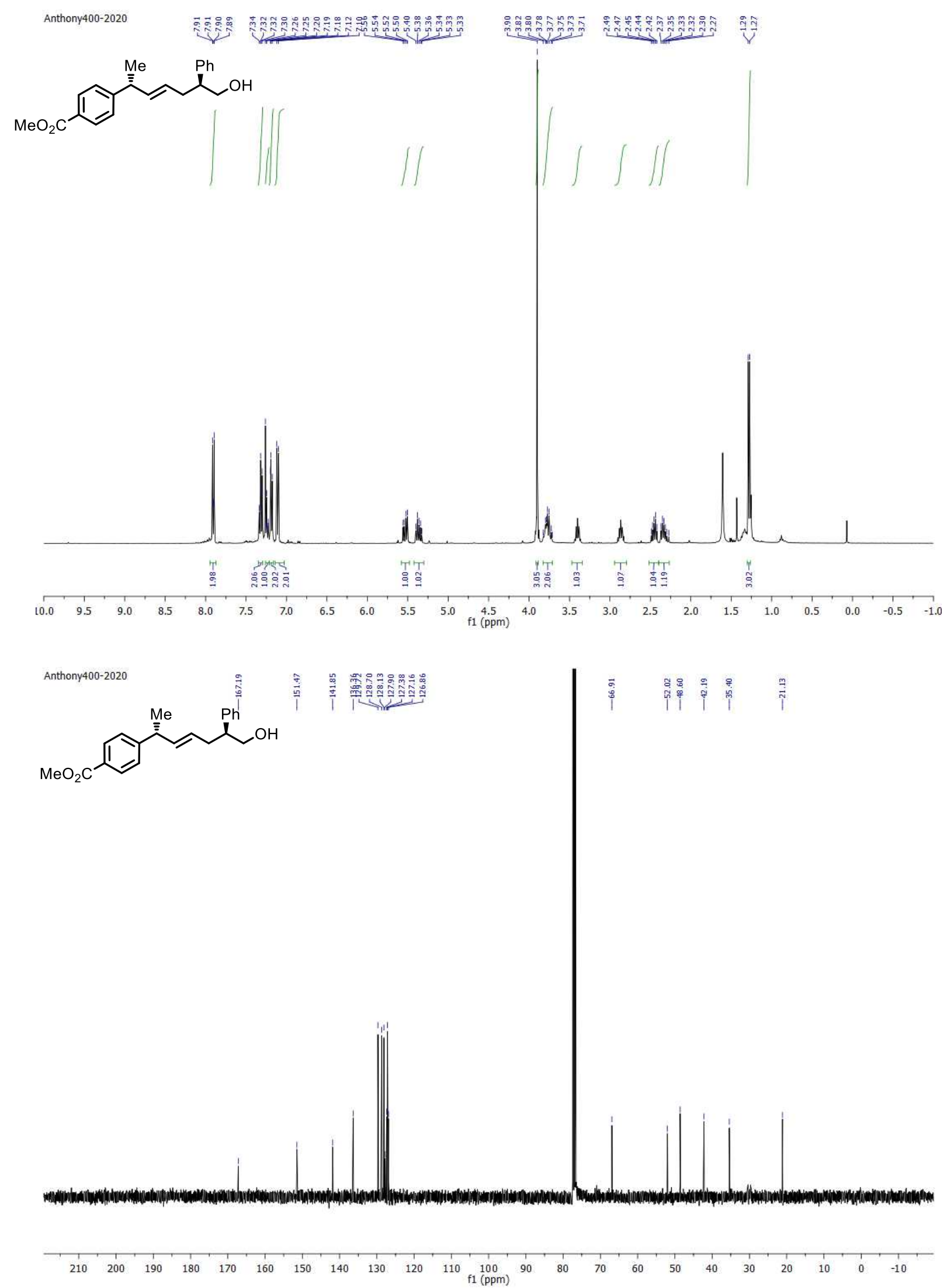
<smiles>COc1ccc([C@H](C#N)/C=C/CC(c2ccccc2)c2ccccc2)cc1OC</smiles>
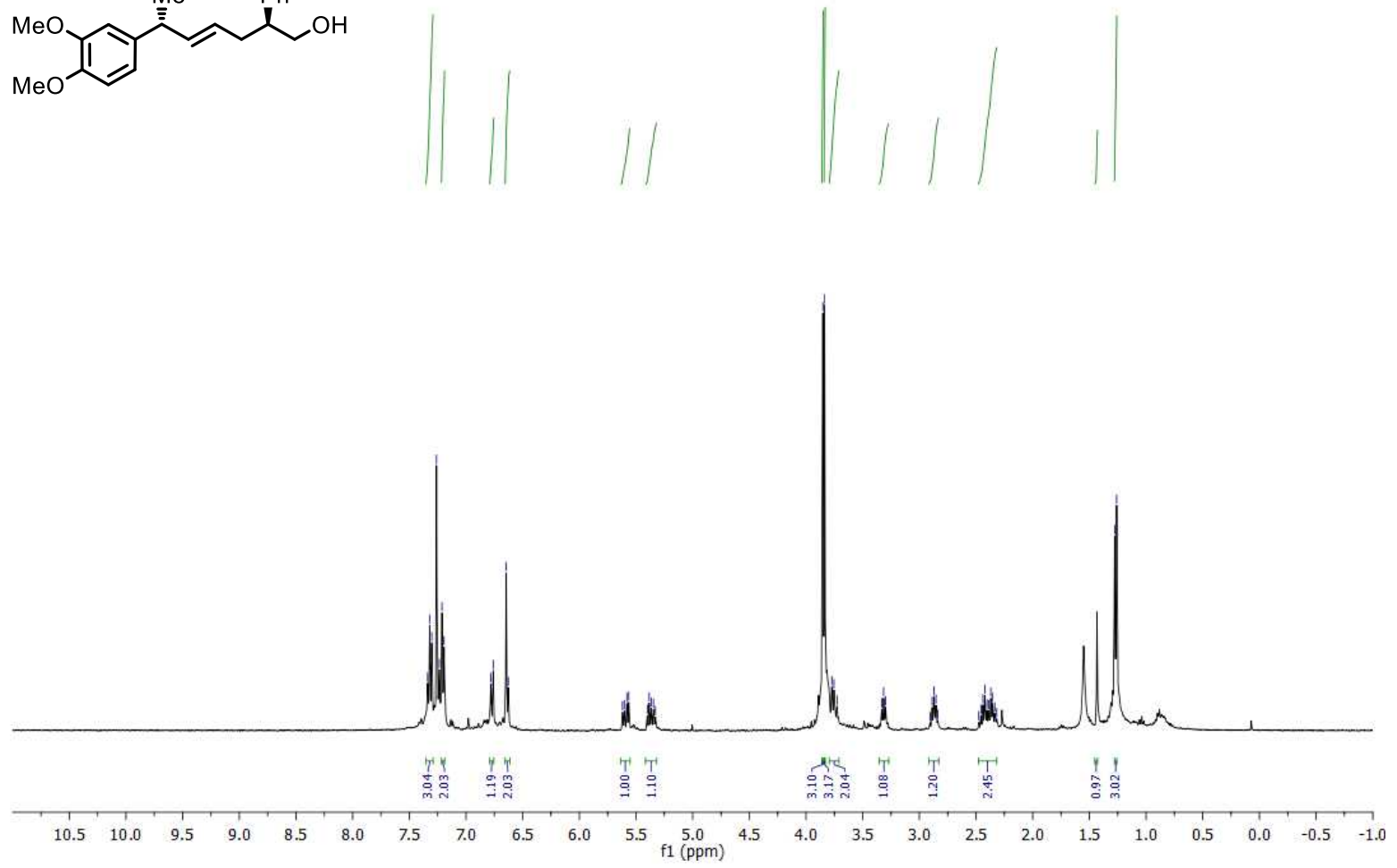

Anthony400-2019
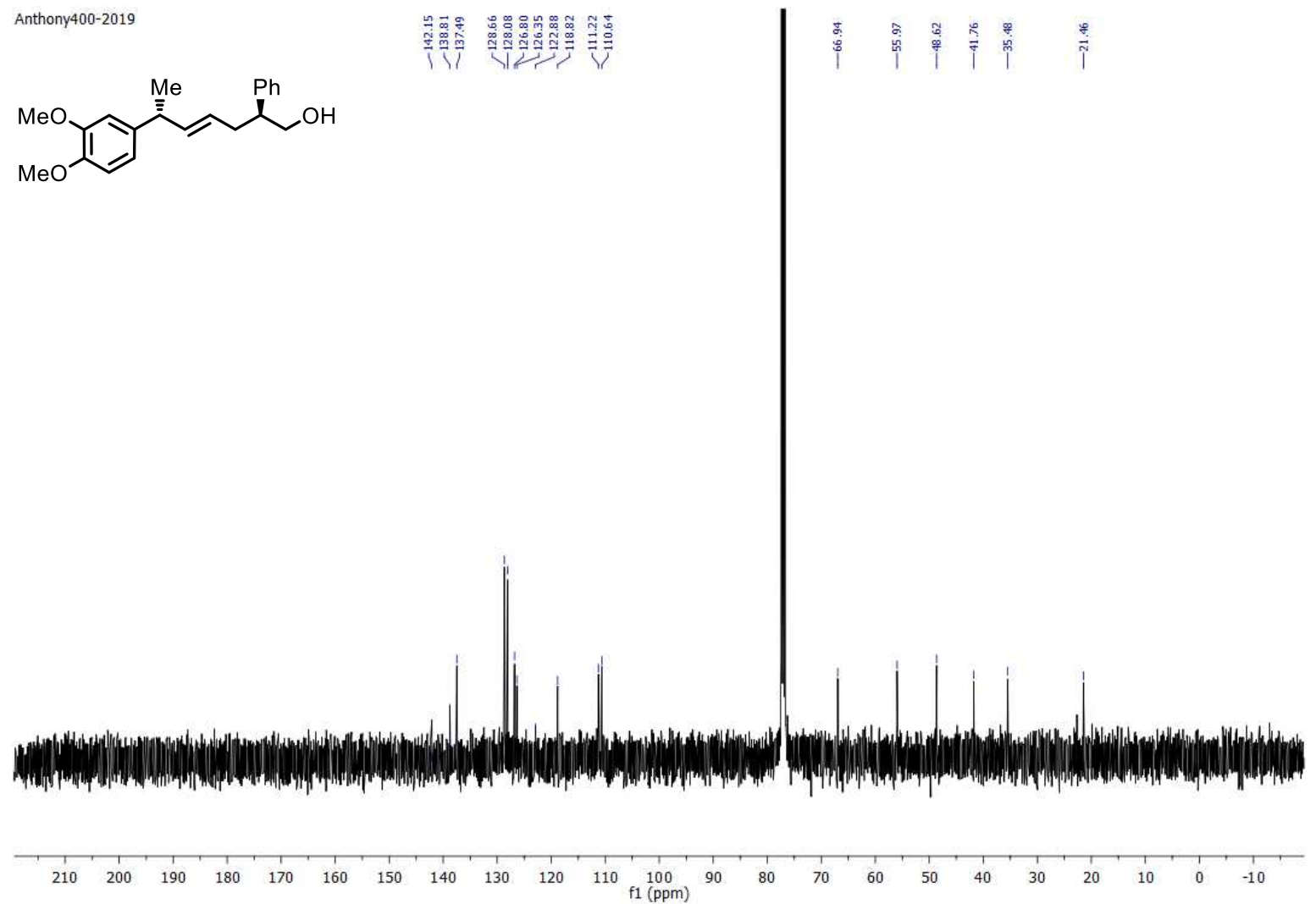
Anthony400-2019

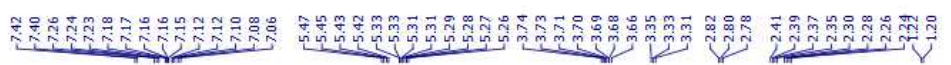

(l)
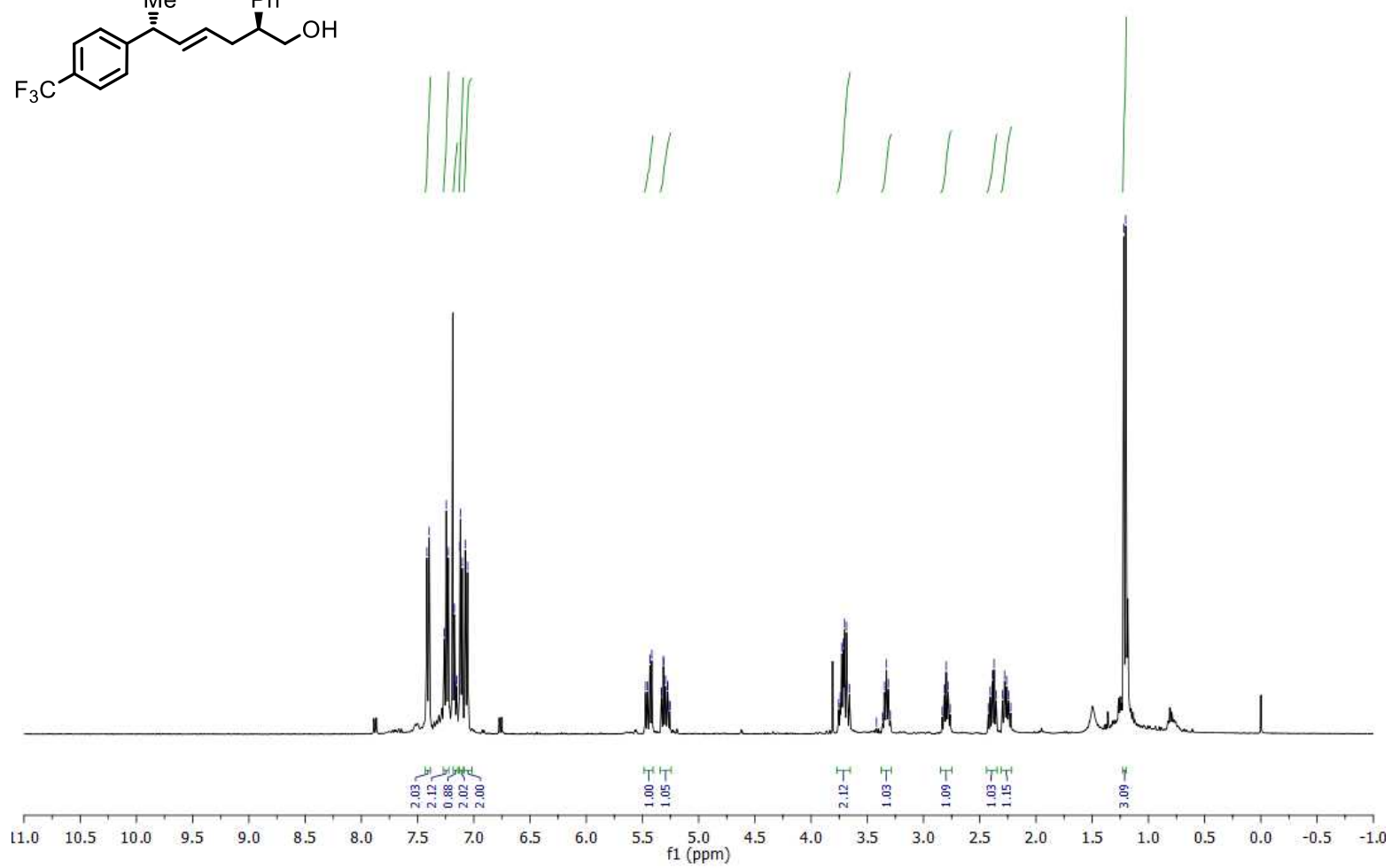

Anthony400-2019<smiles>C[C@H](/C=C/CC(CO)c1ccccc1)c1ccc(C(F)(F)F)cc1</smiles>
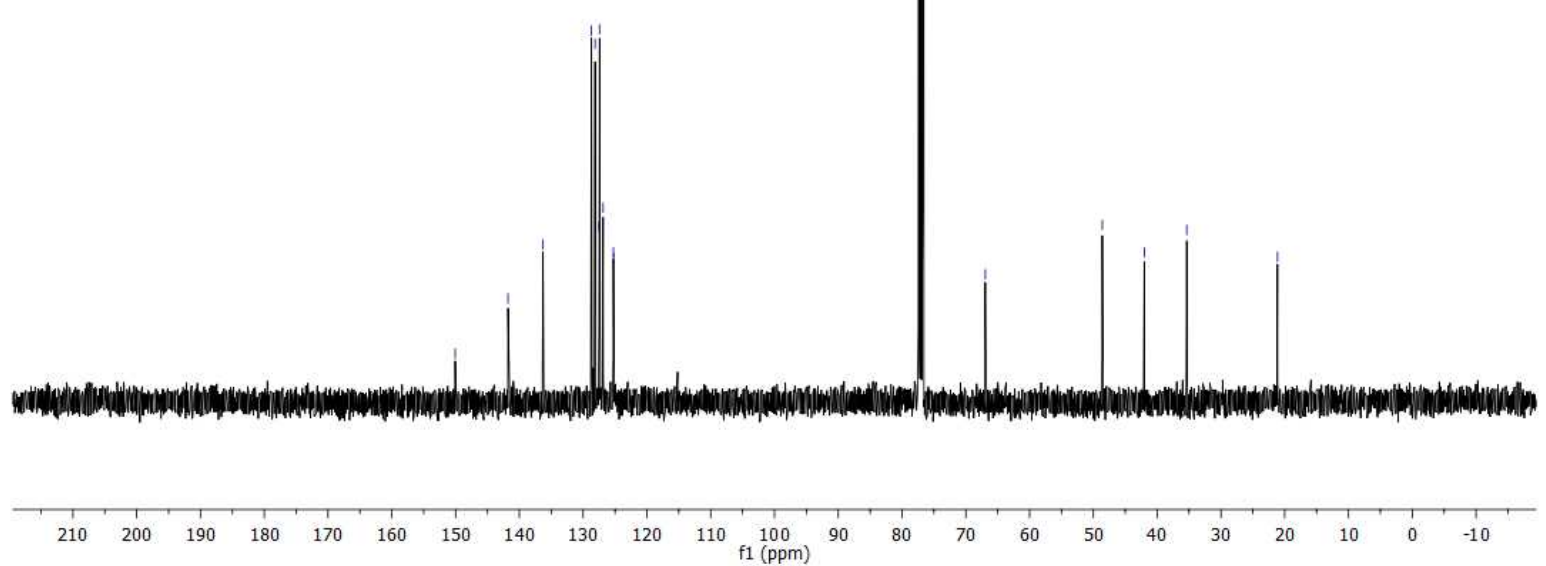


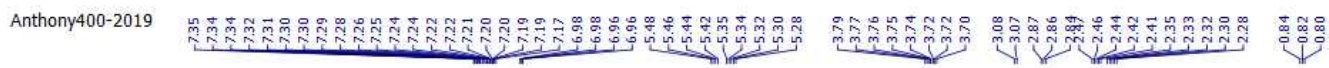
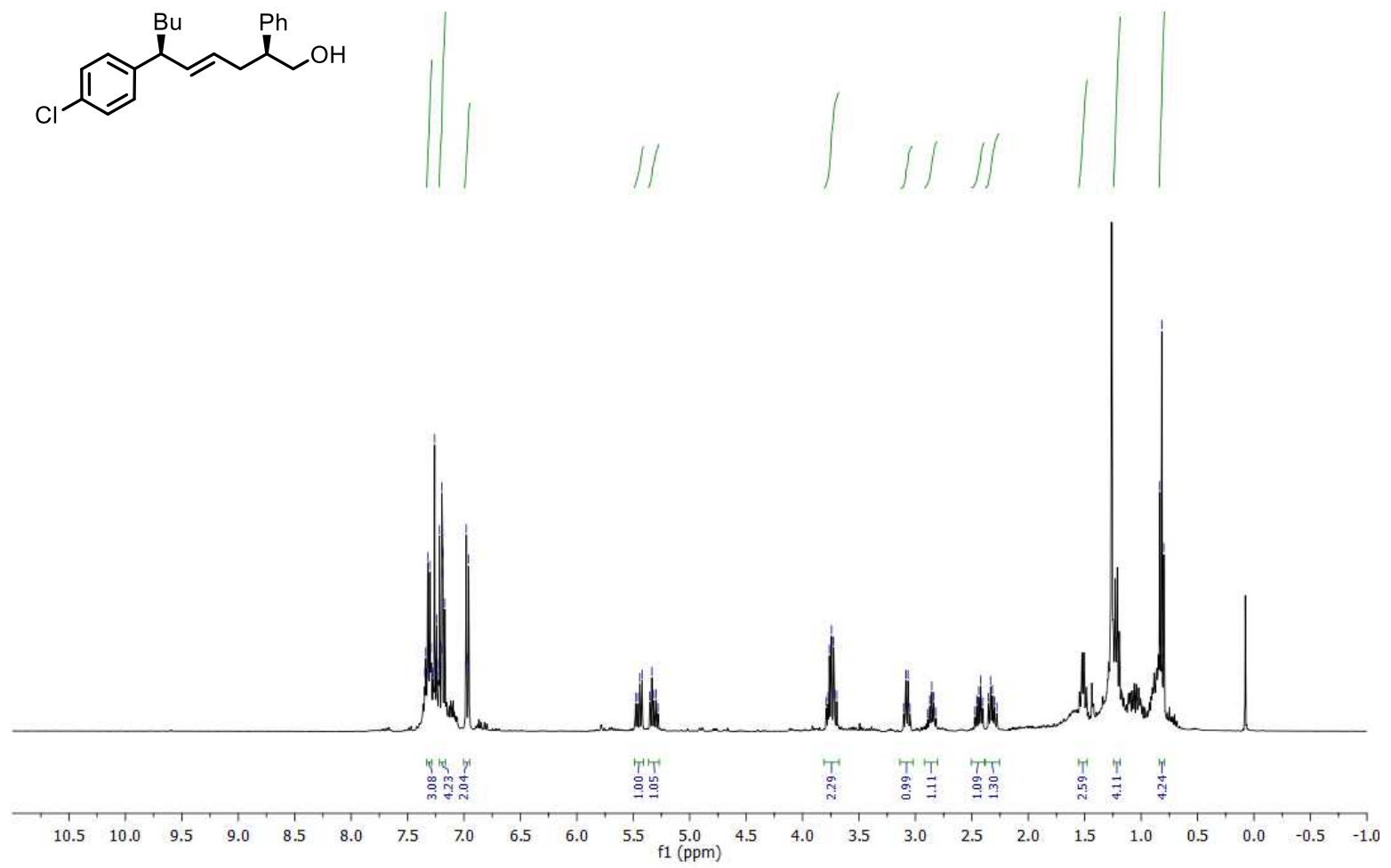

Anthony400-2019<smiles>OC(CC=CC(=CCC(O)c1ccc(Cl)cc1)c1ccccc1)c1ccccc1</smiles> 


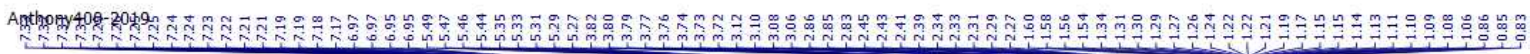<smiles>OCC(CC=C[C@@H]([10B]c1ccc(Cl)cc1)c1ccccc1)c1ccccc1</smiles>
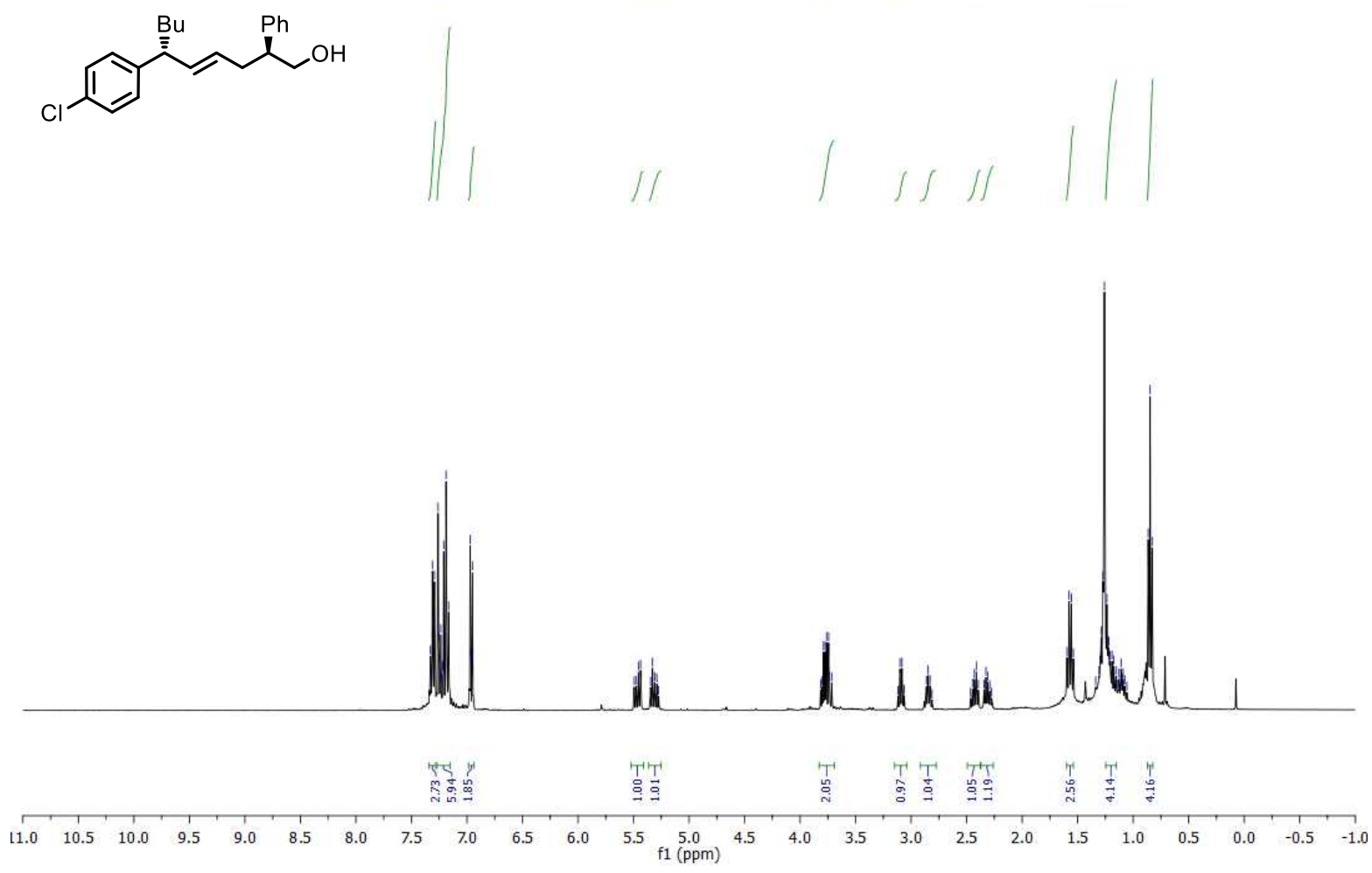

Anthony400-2019

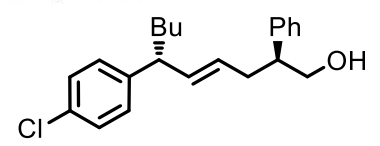

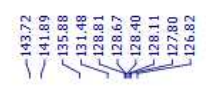
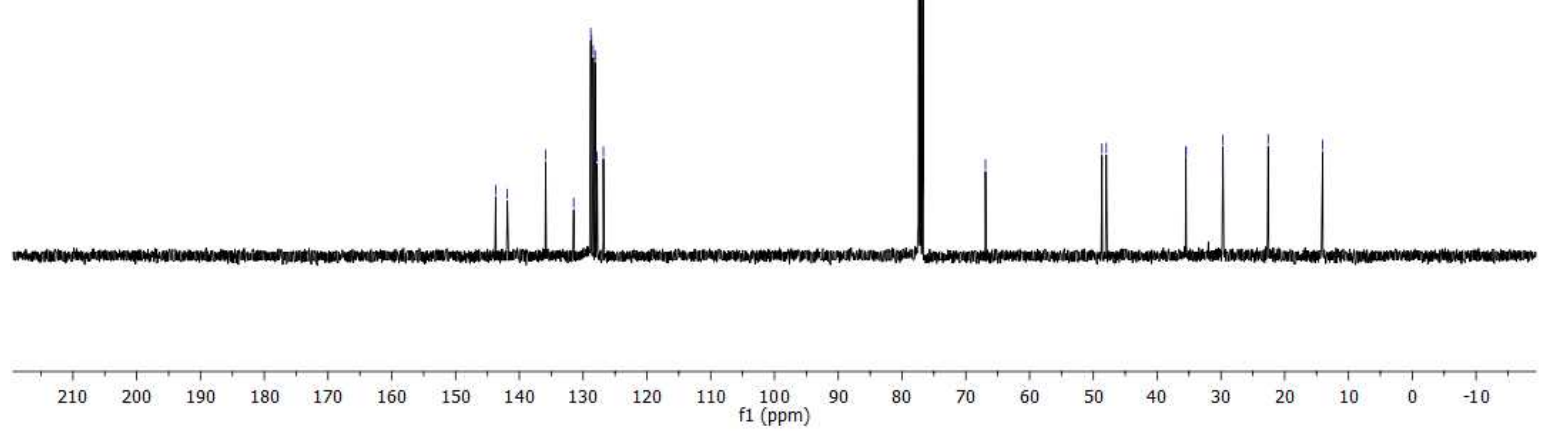

S48 

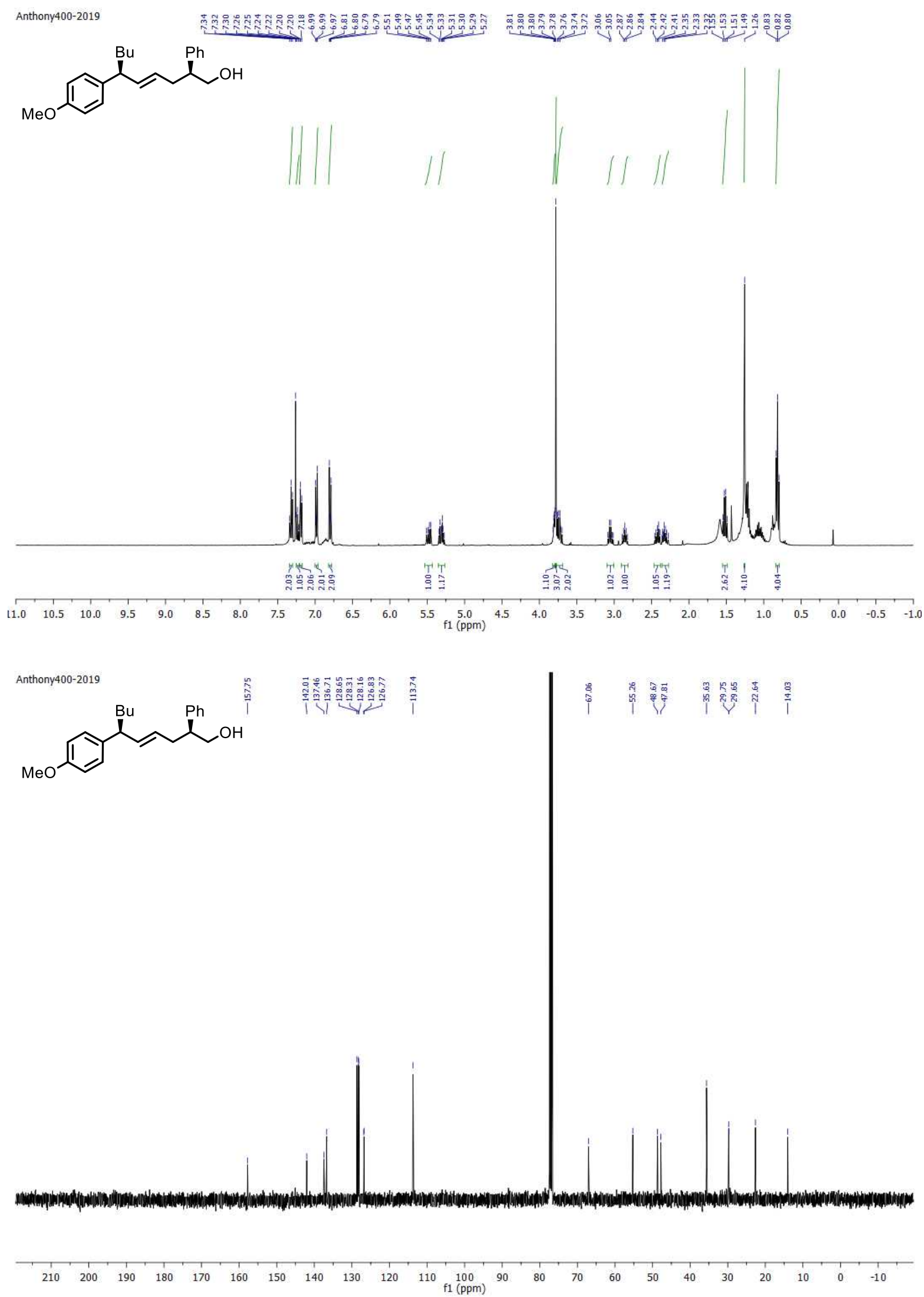


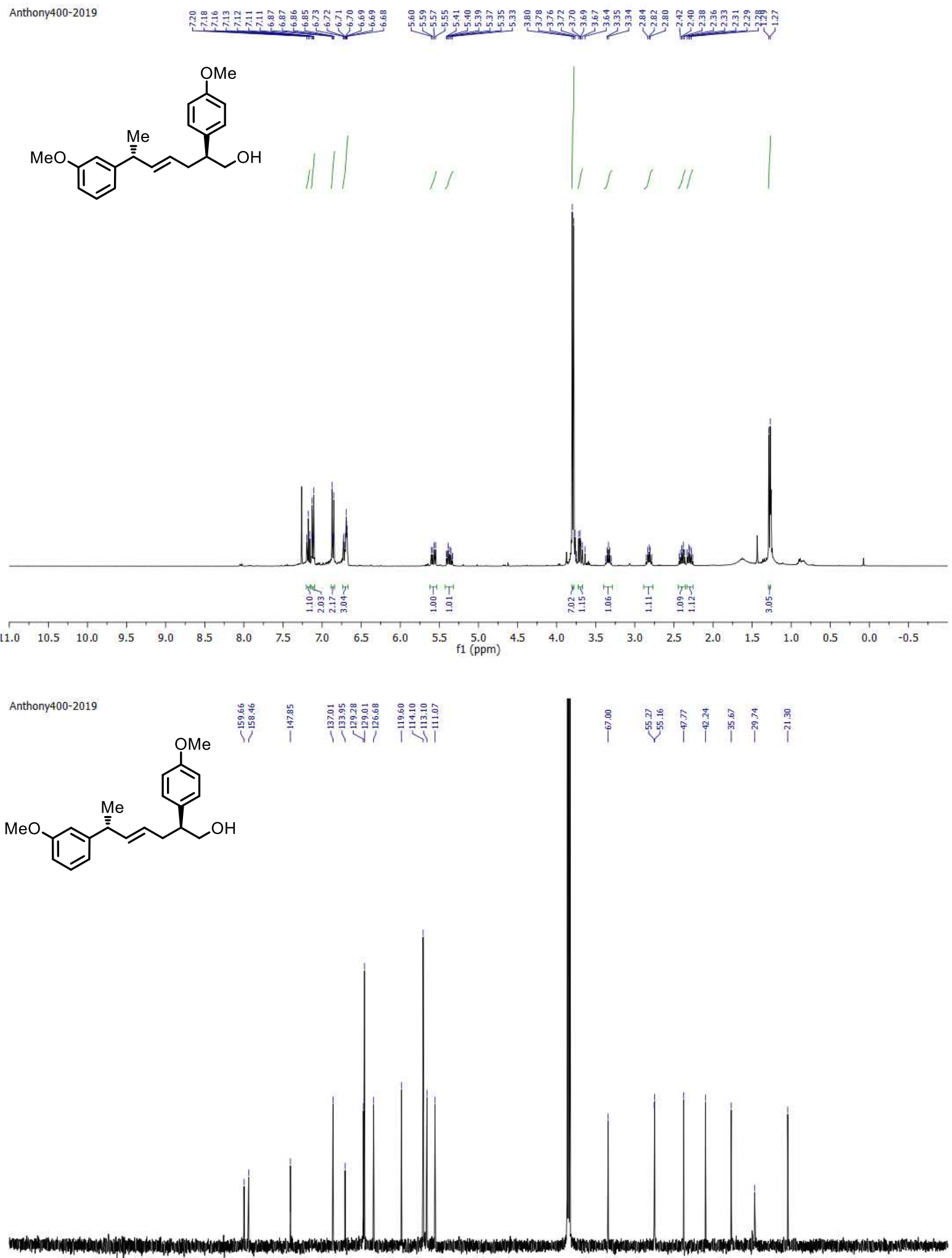

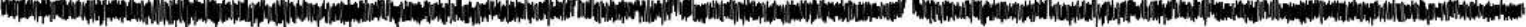

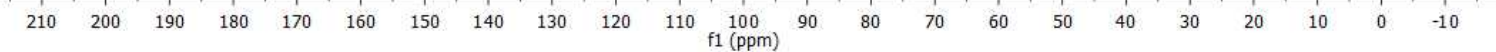



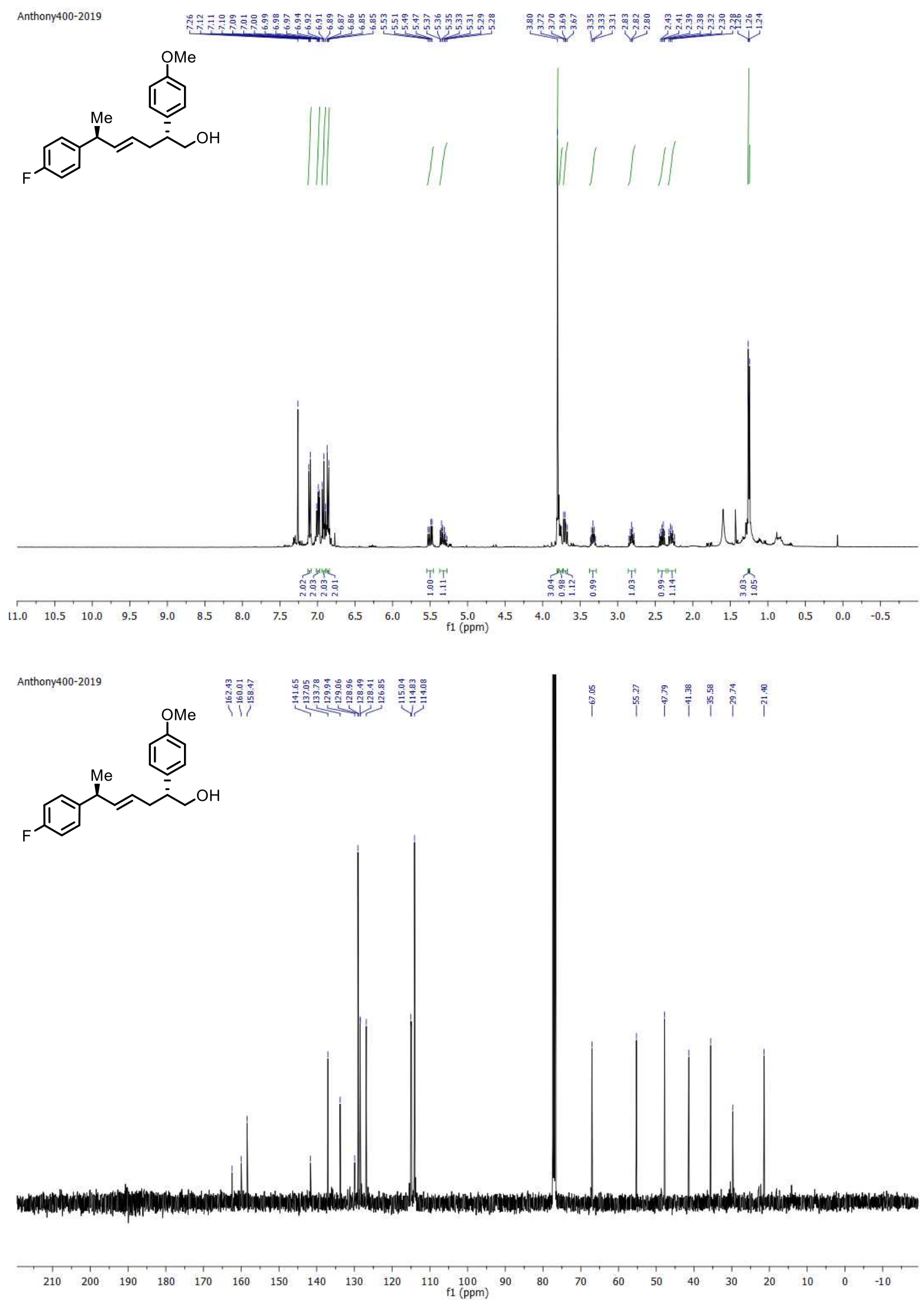
<smiles>COc1ccc(C(C)(C)c2ccc(OC)cc2)cc1</smiles>
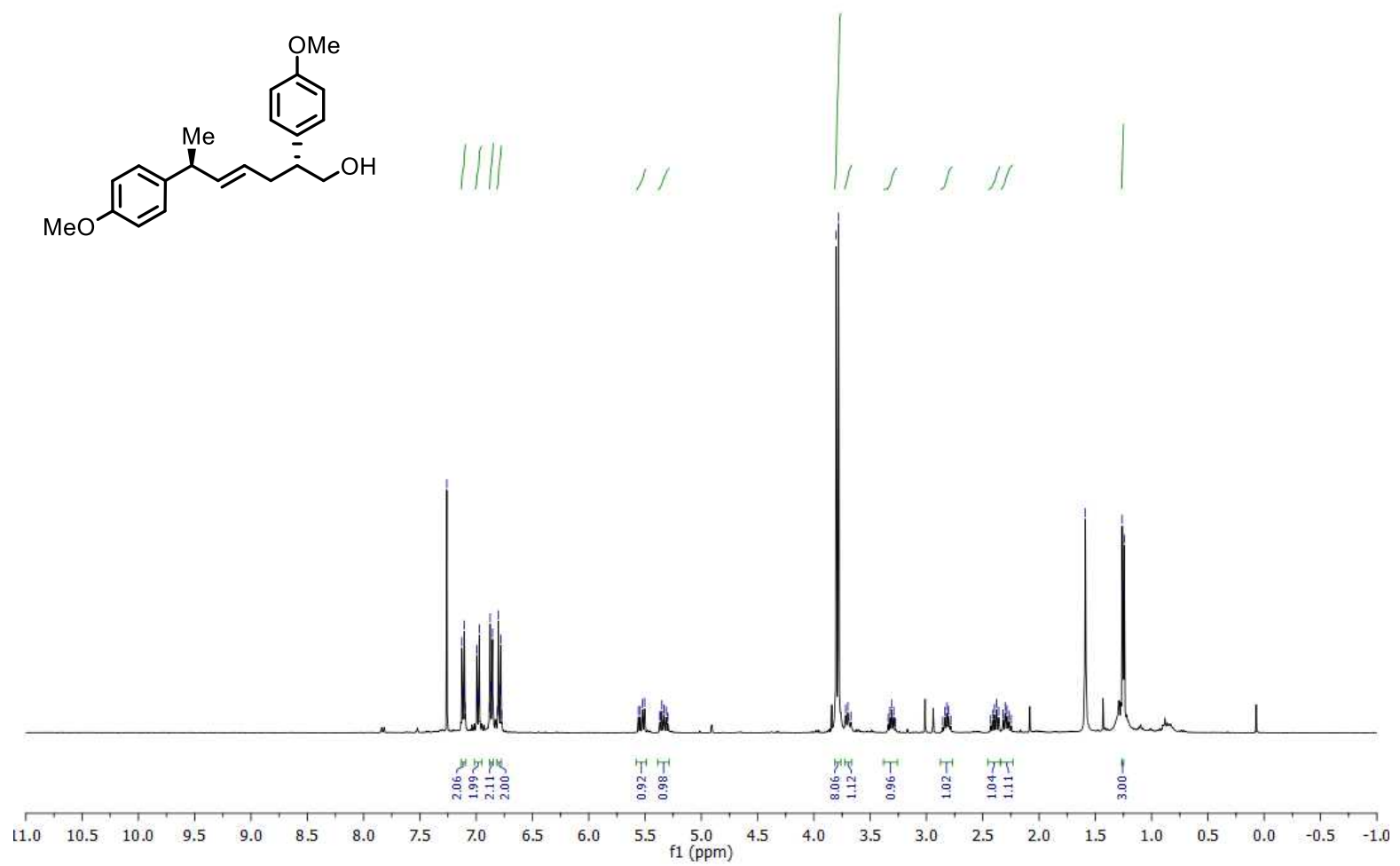

Anthony400-2019<smiles>COc1ccc([C@@H](CO)C/C=C/C(C)(C)c2ccc(OC)cc2)cc1</smiles>

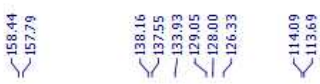

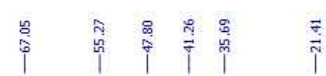

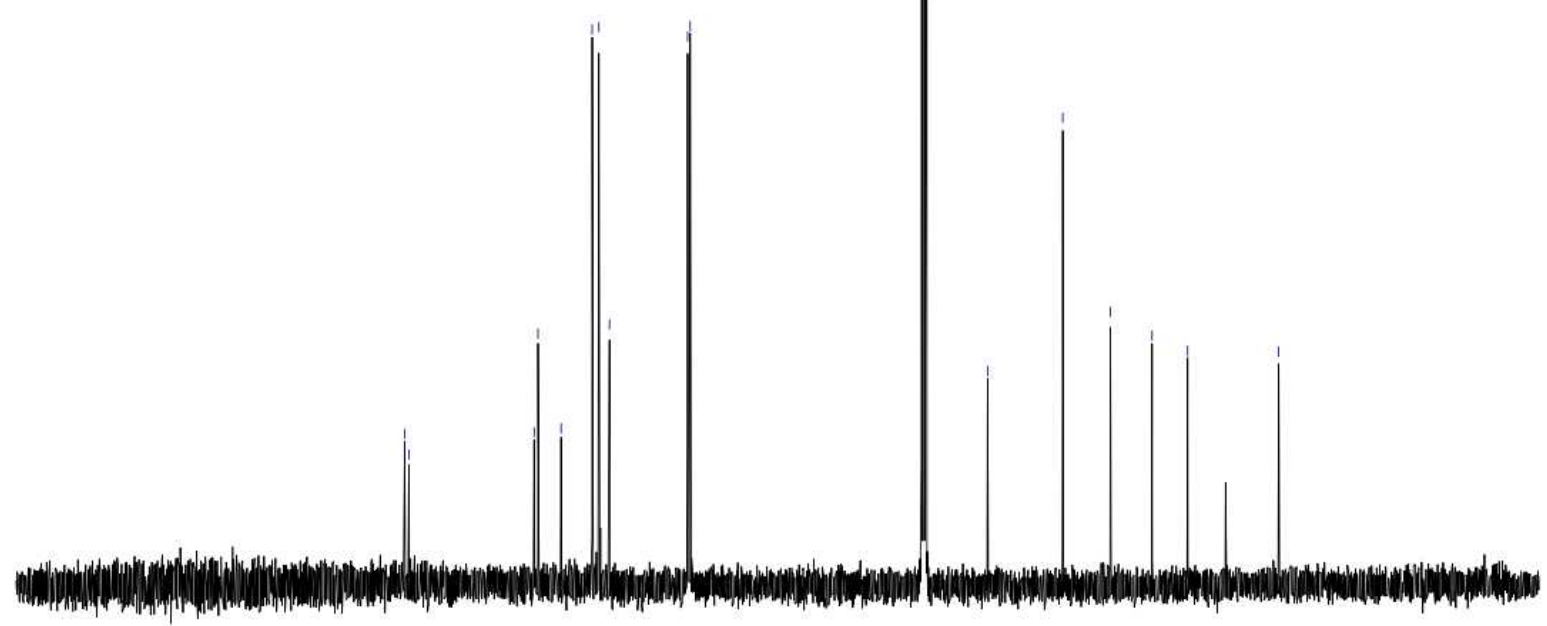

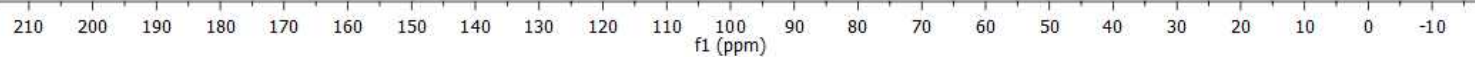



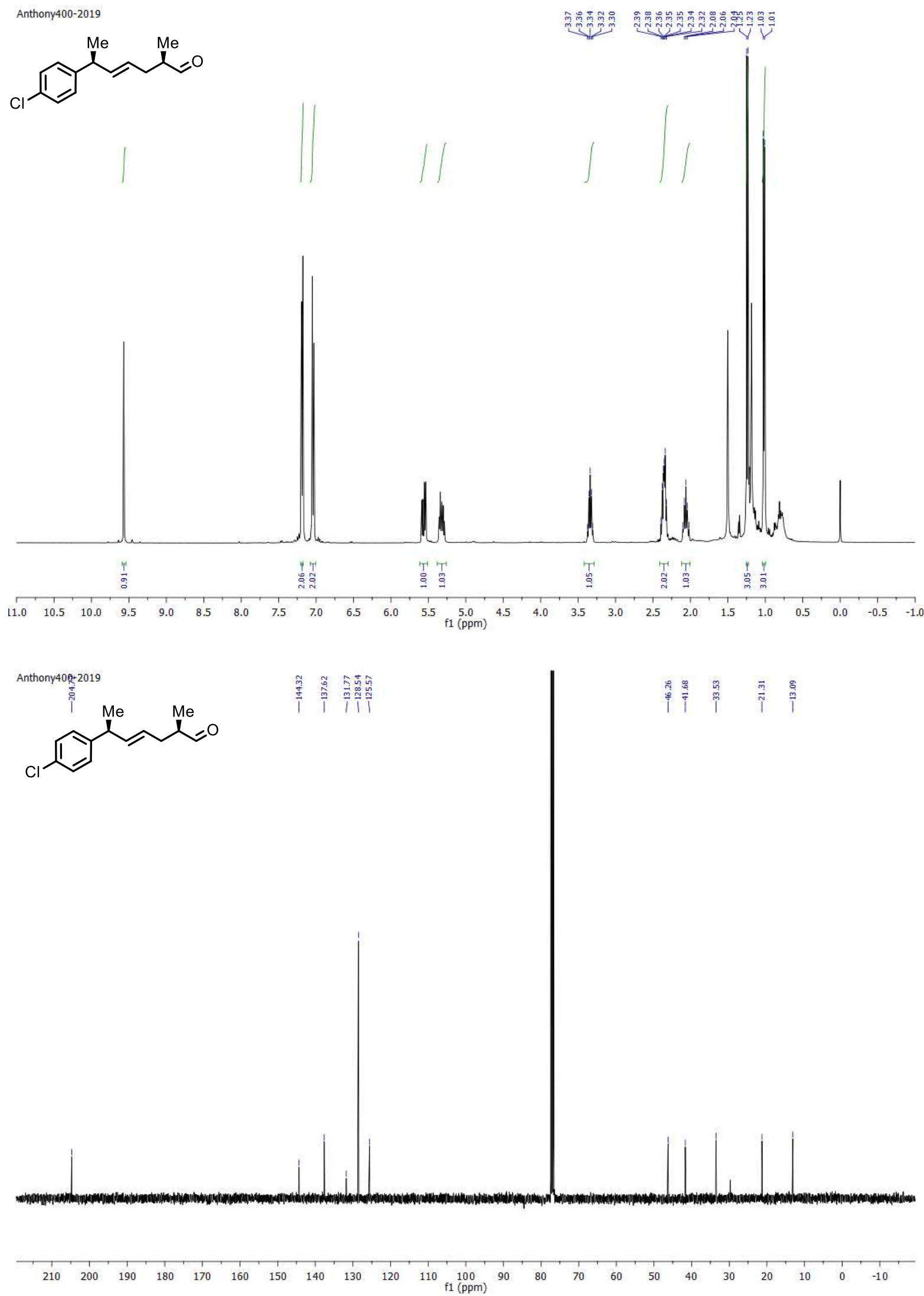

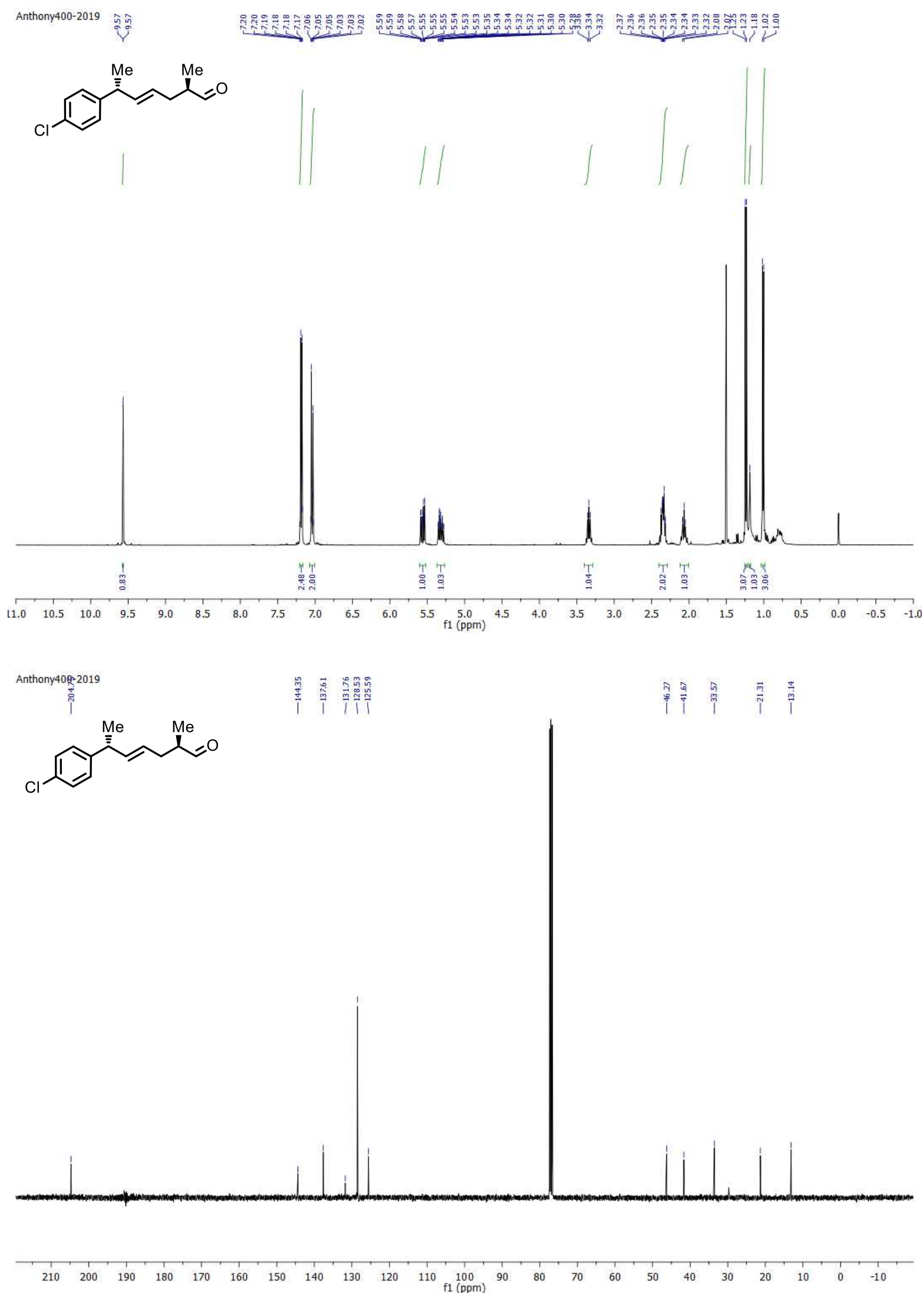

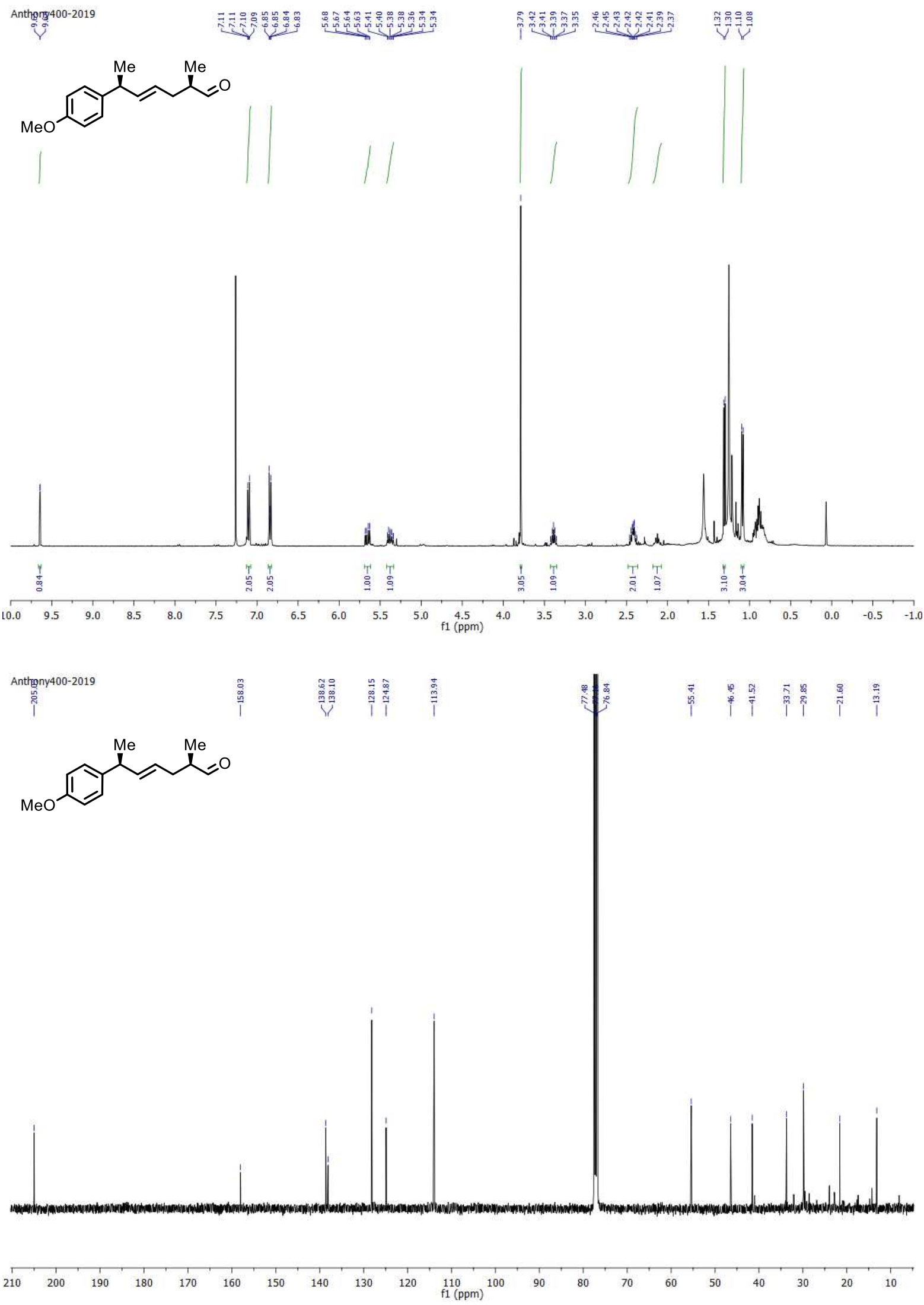

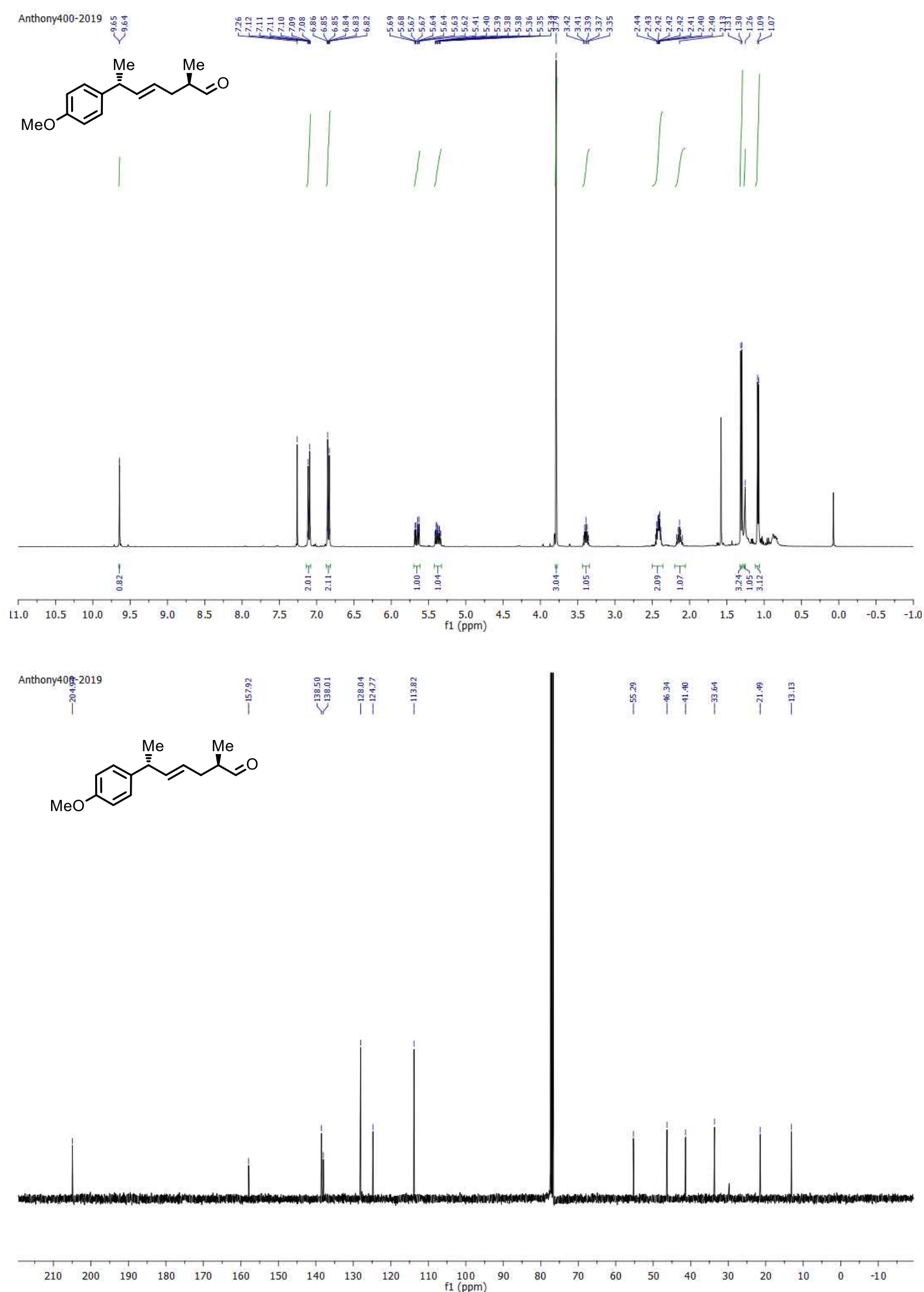

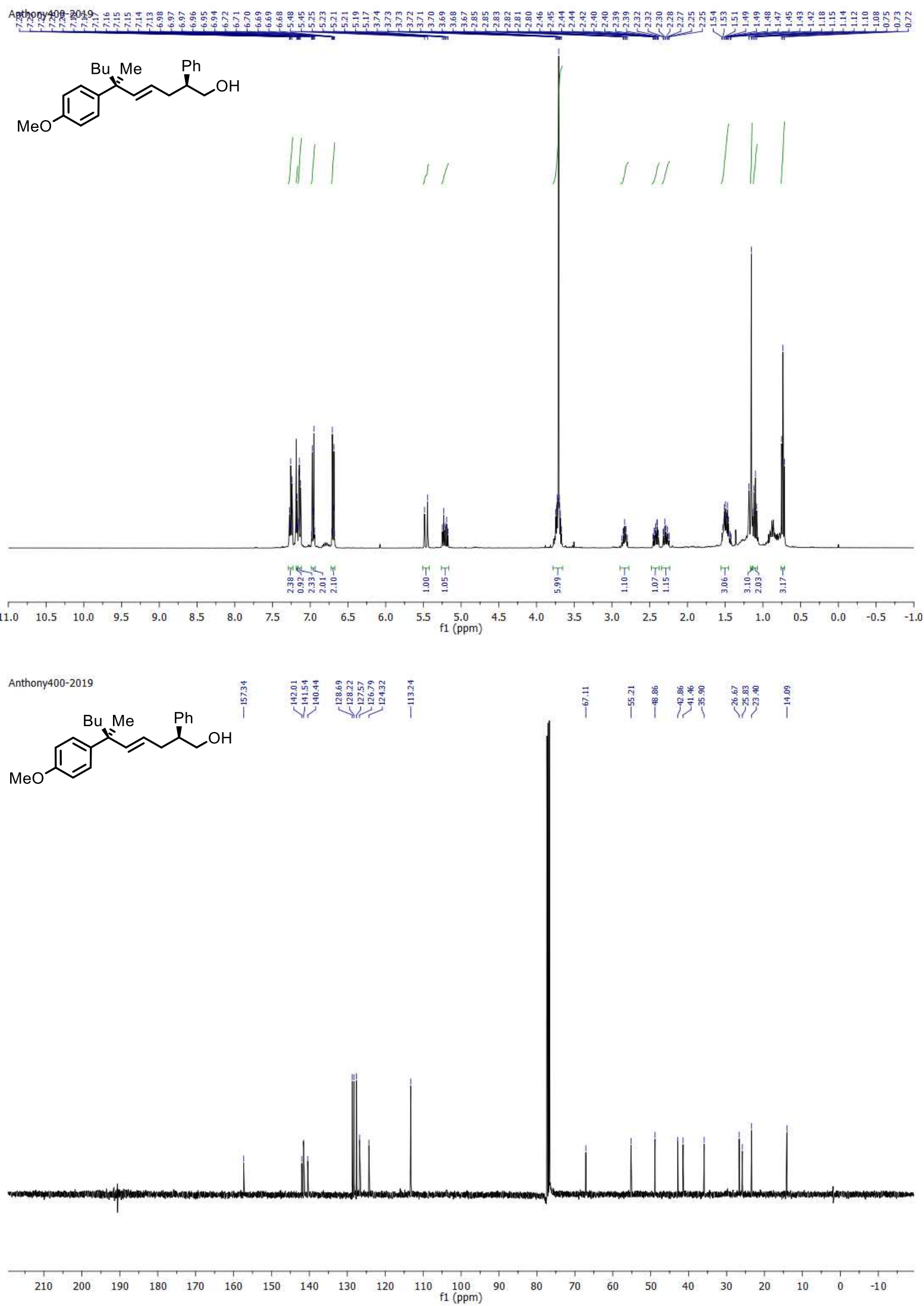

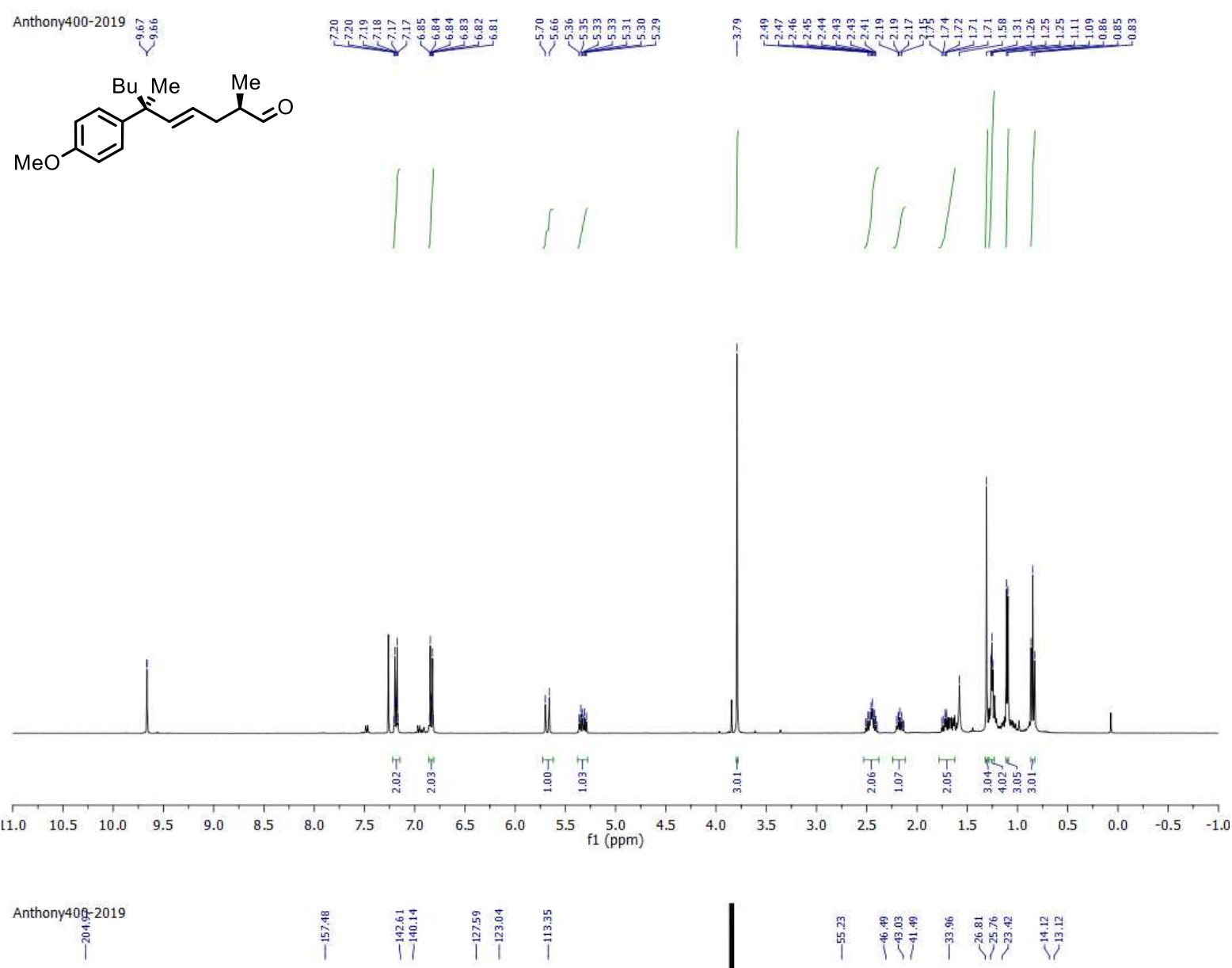
=
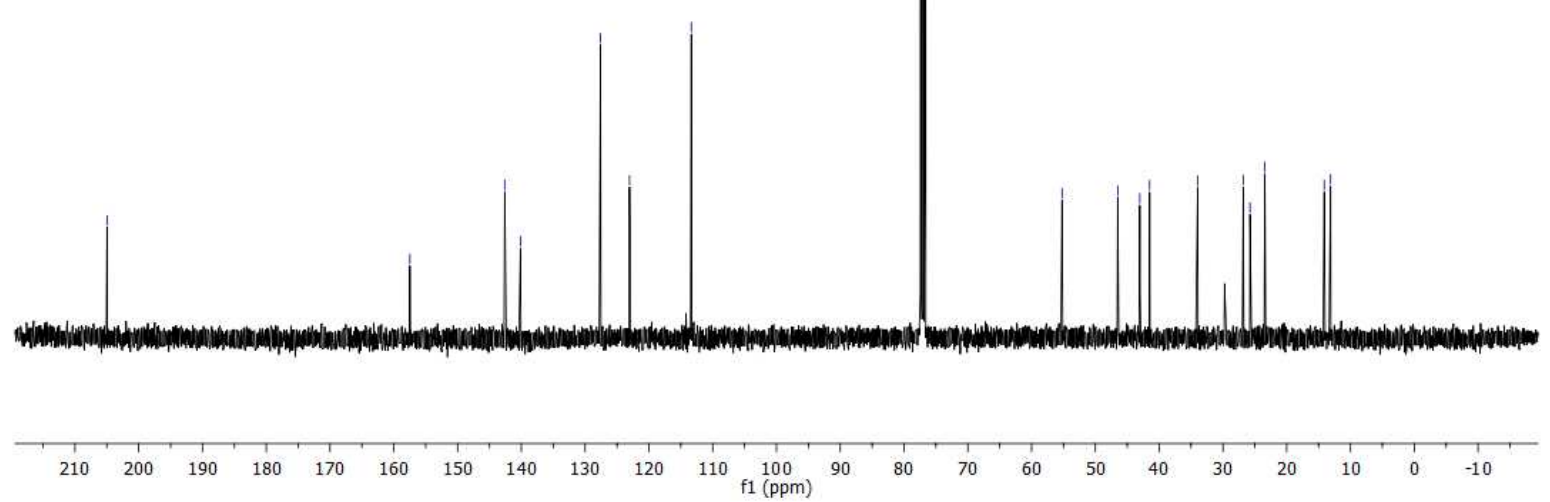


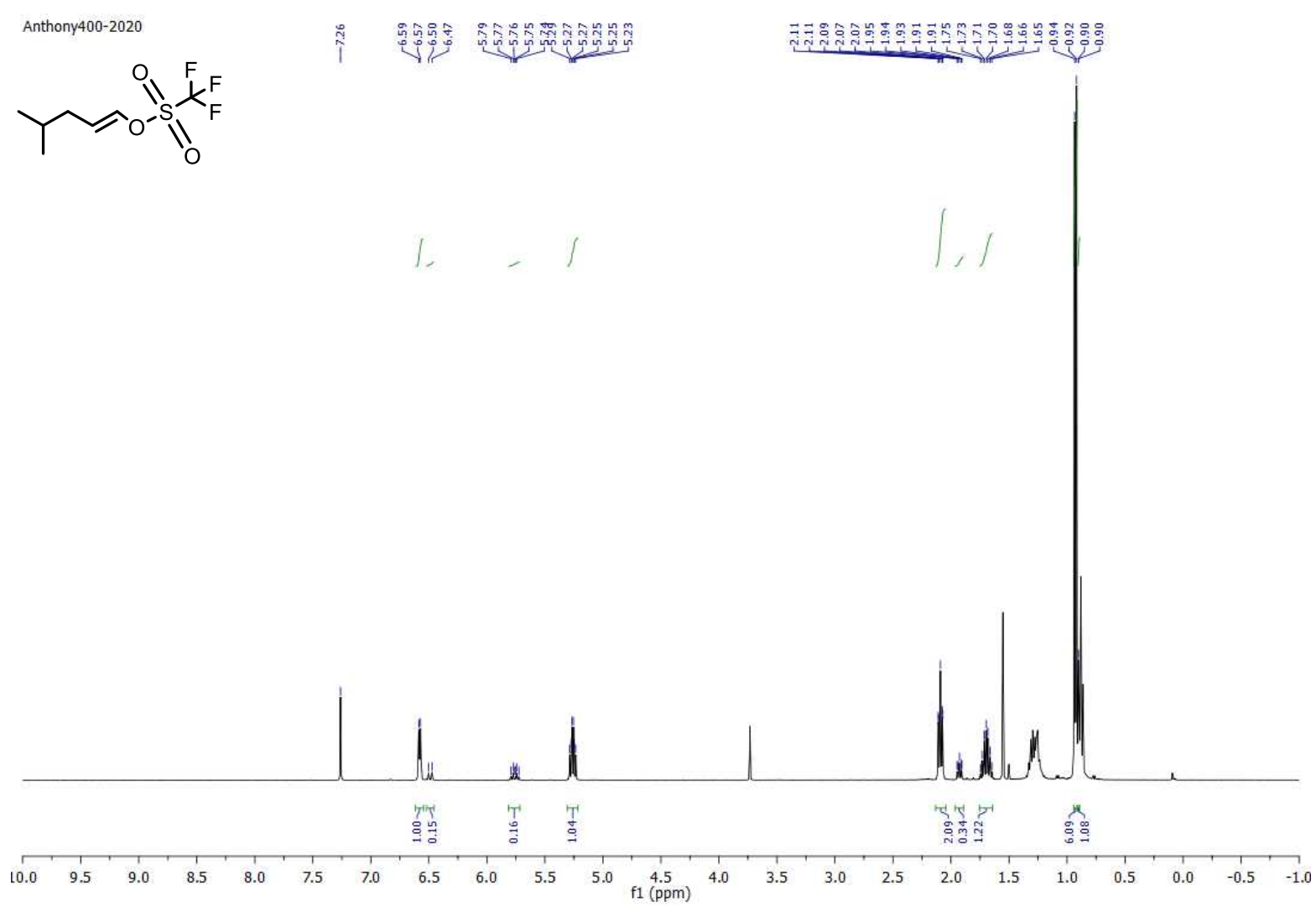

Anthony400-2020<smiles>CC(C)CC=COS(=O)(=O)C(F)(F)F</smiles> 


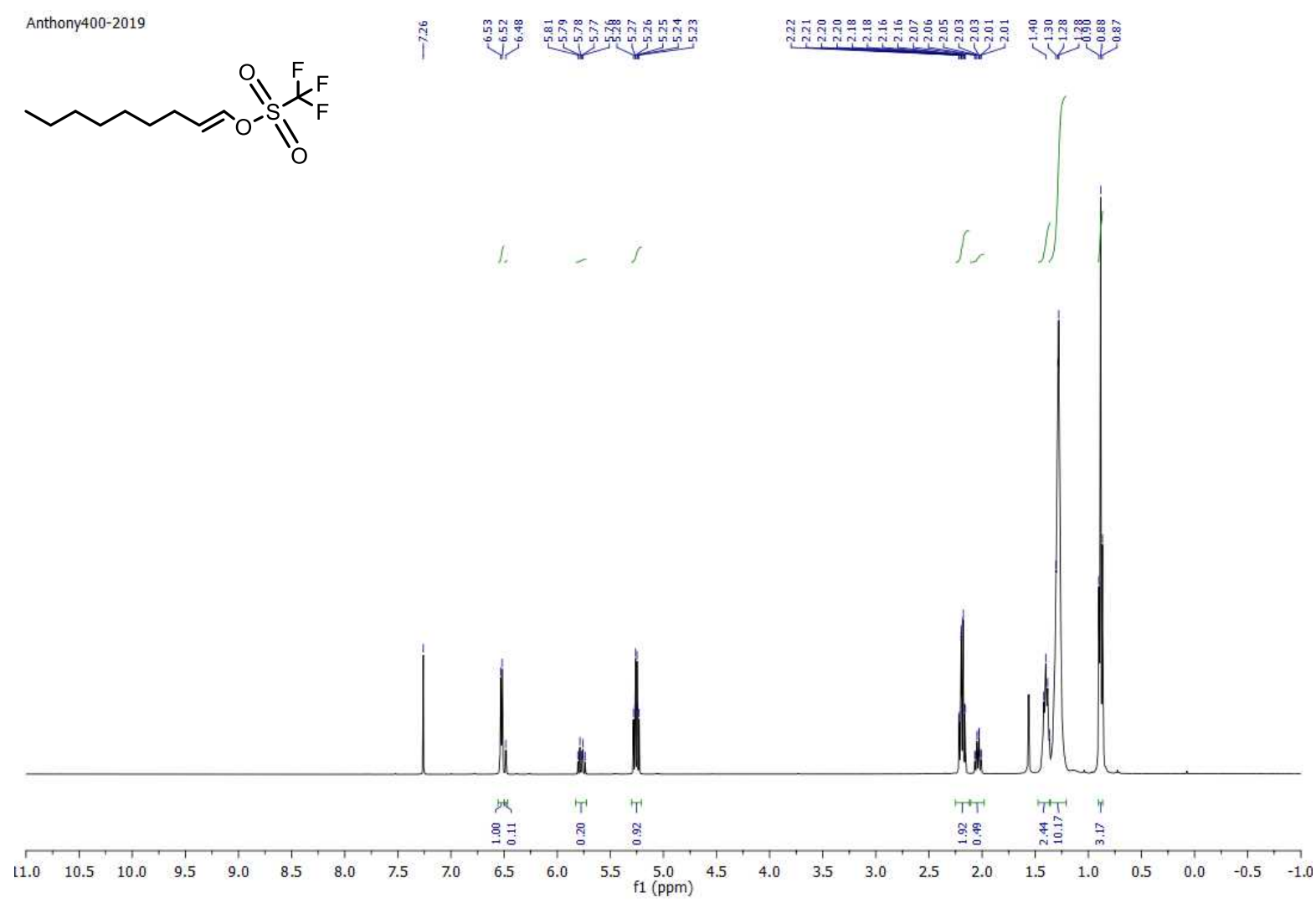

Anthony400-2019

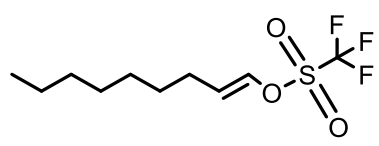
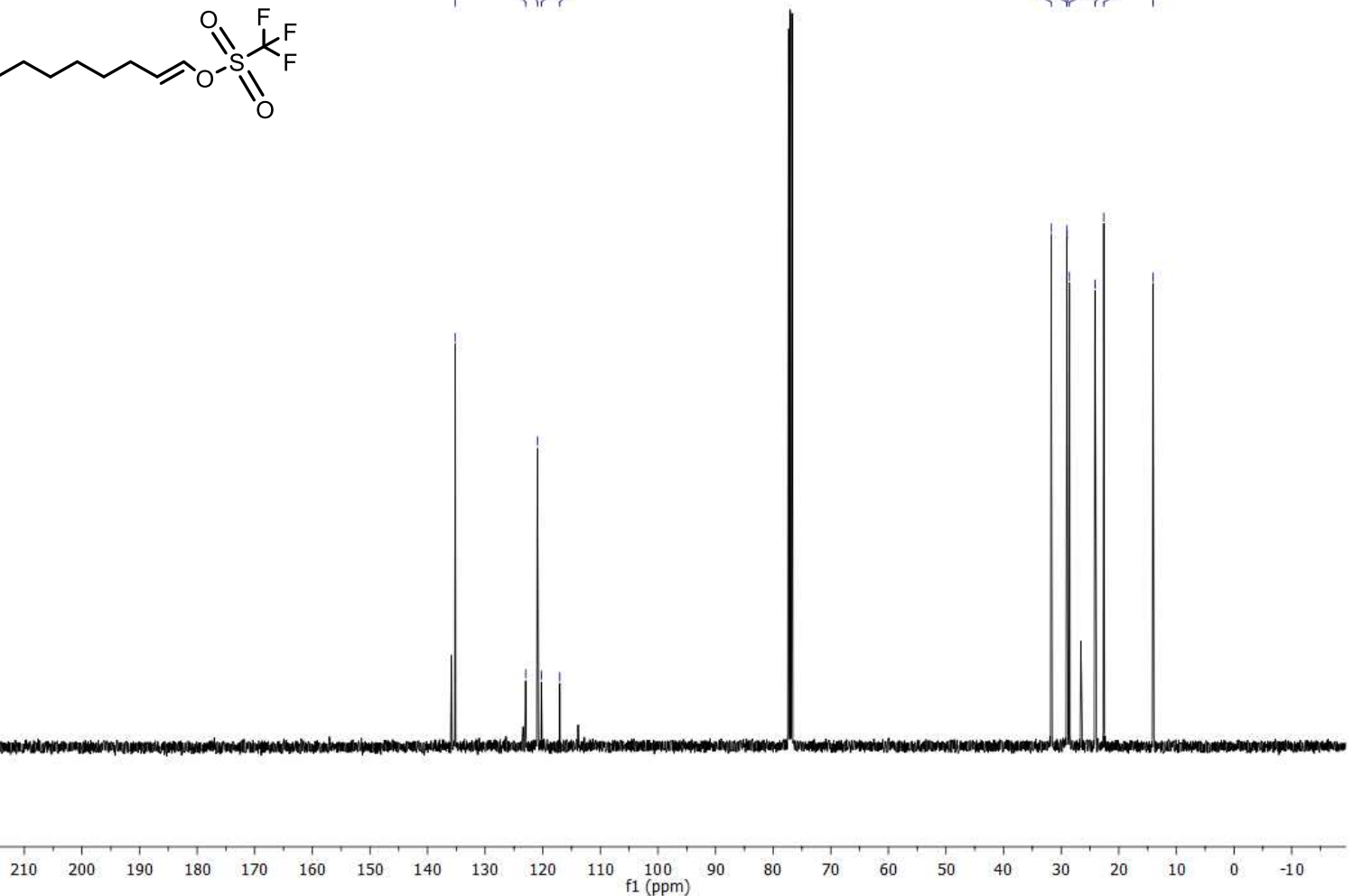


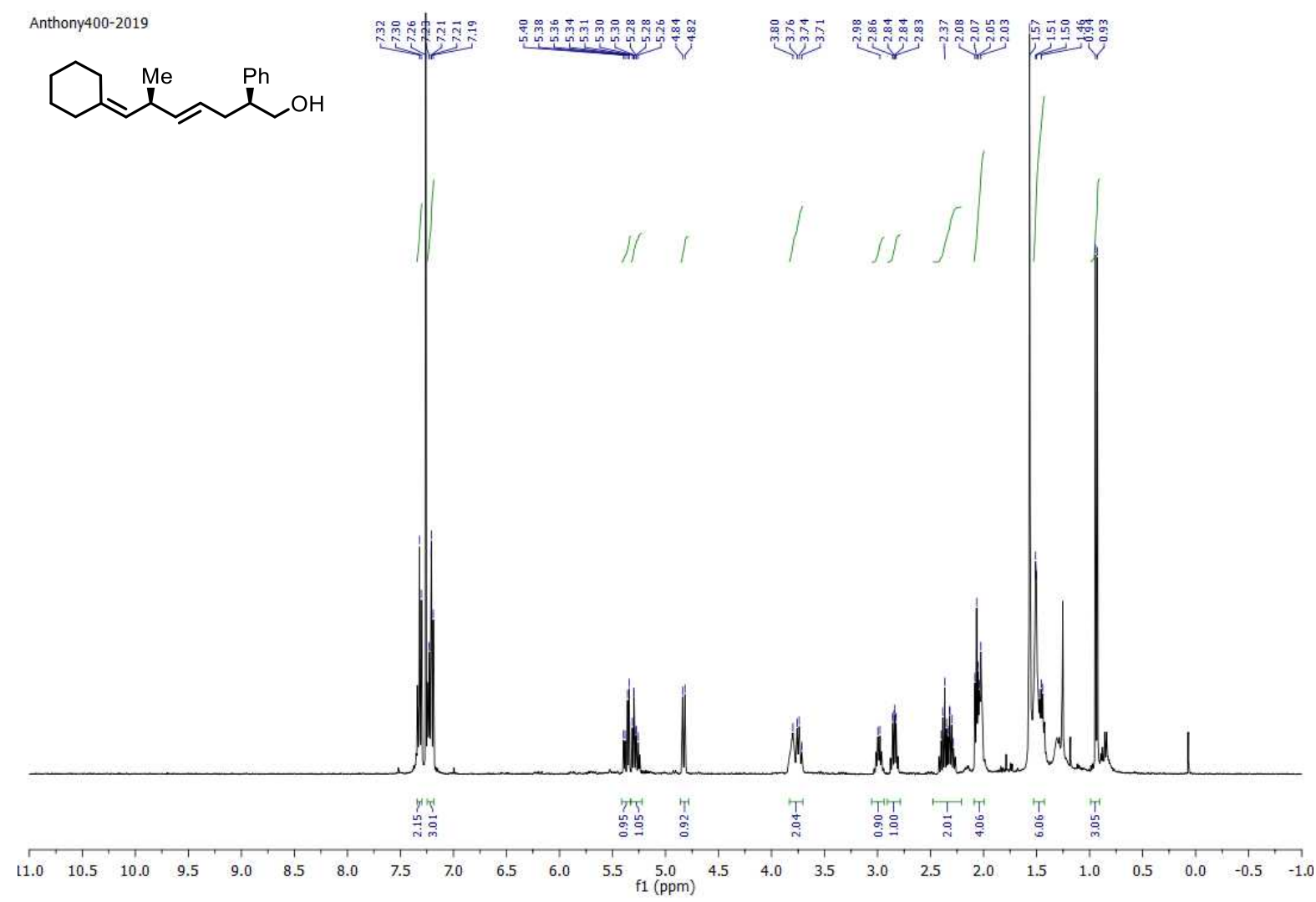

Anthony400-2019

Quecheron 
Anthon400-2019

事
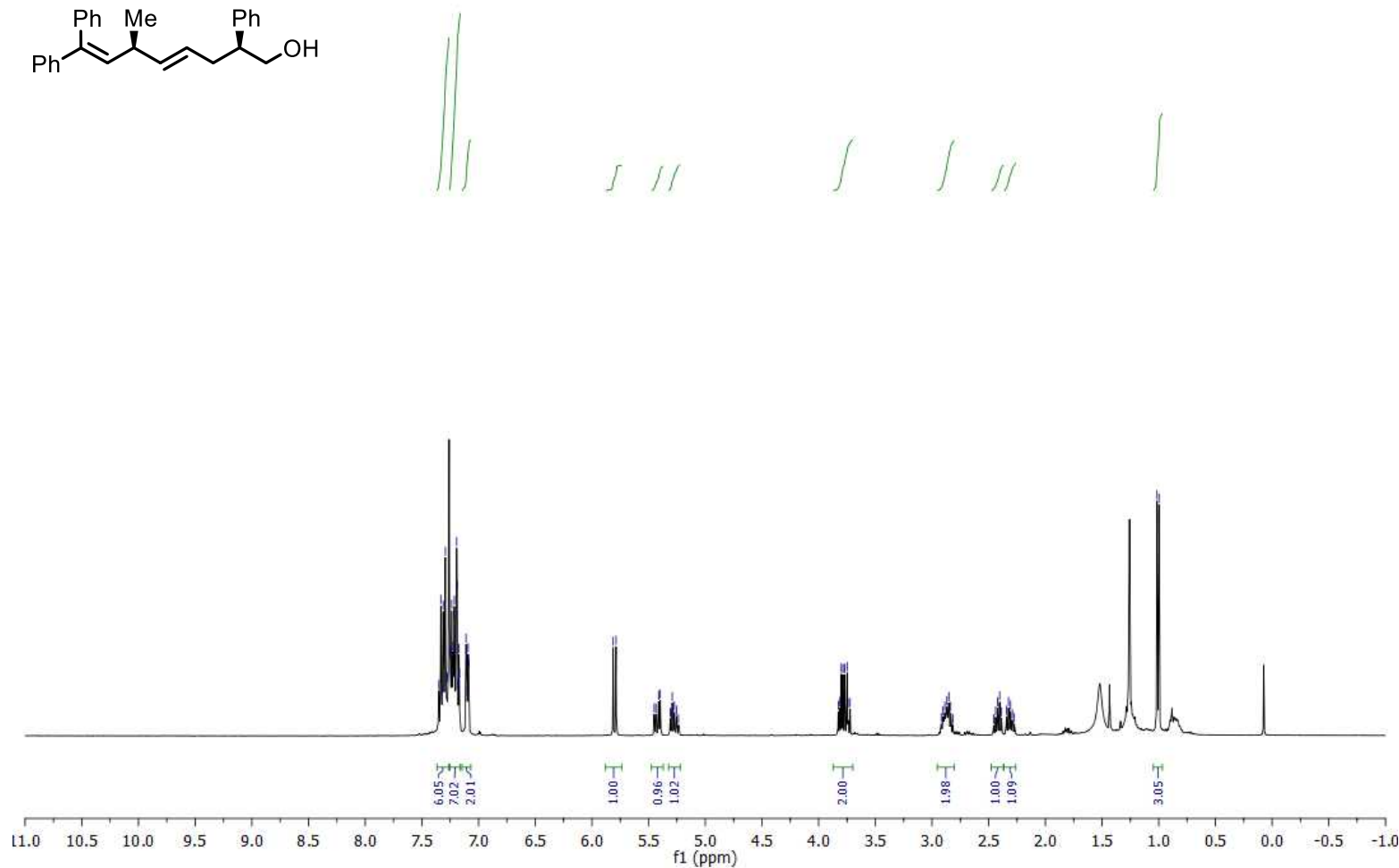

Anthony400-2019

( 
(lept

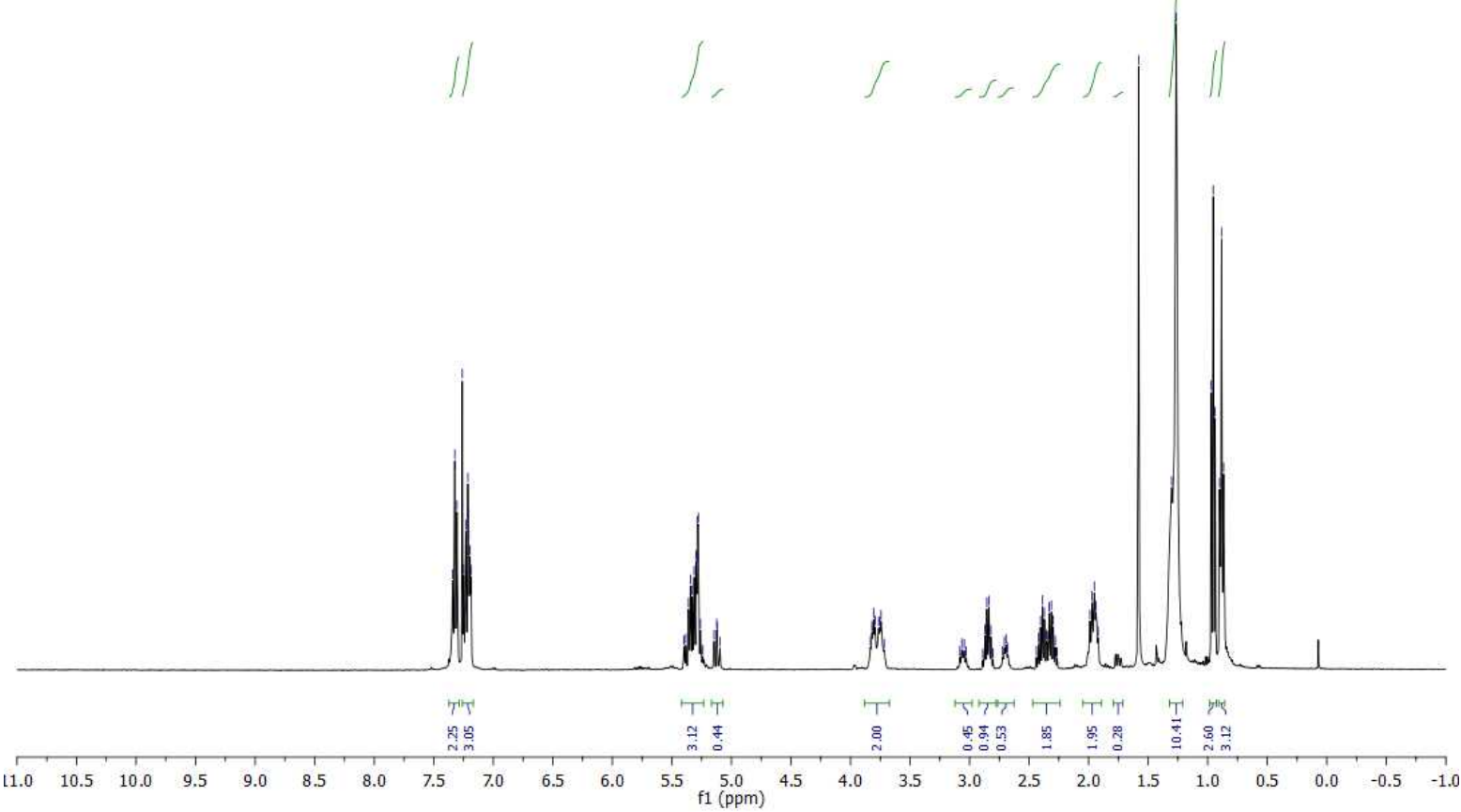

Anthony400-2019

(lept

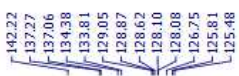

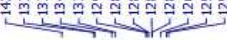
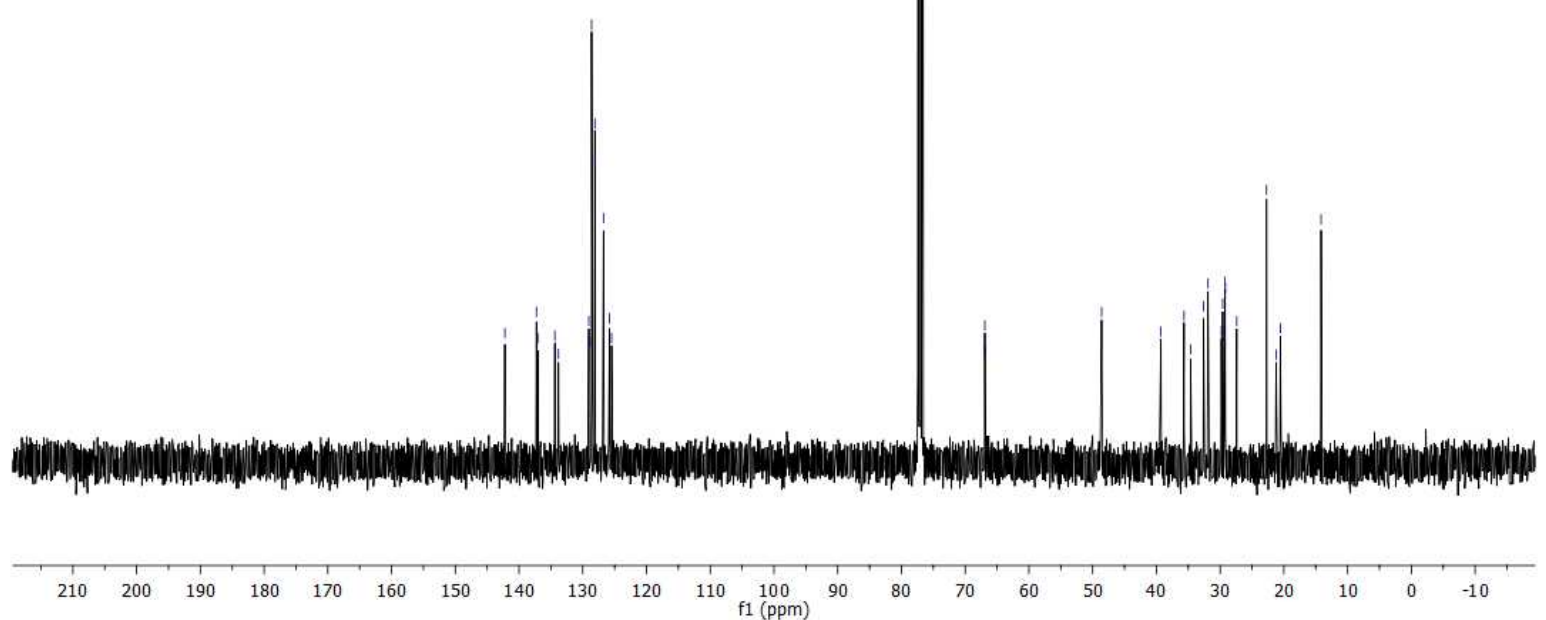


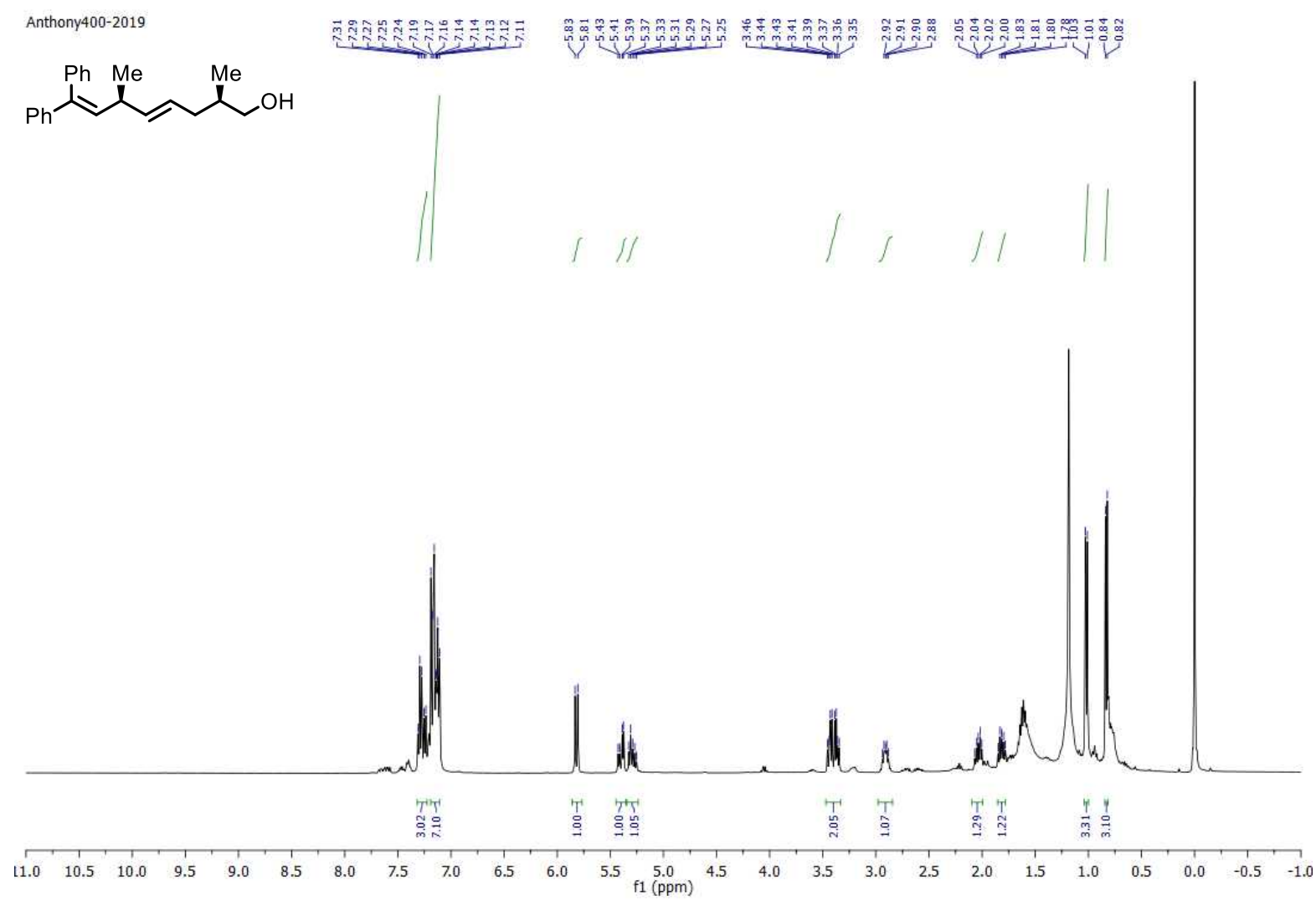

Anthony400-2019<smiles>CC(C=CCC(C)CO)C=CC(=O)c1ccccc1</smiles>

ClOH

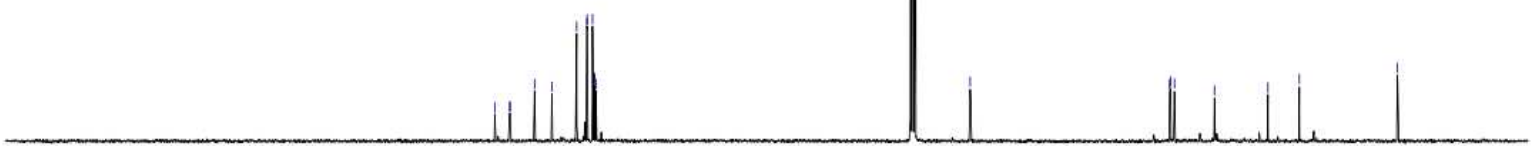

$\begin{array}{lllllllllllllllllllllll}210 & 200 & 190 & 180 & 170 & 160 & 150 & 140 & 130 & 120 & 110 & \begin{array}{c}100 \\ \mathrm{f} 1(\mathrm{ppm})\end{array} & 90 & 80 & 70 & 60 & 50 & 40 & 30 & 20 & 10 & 0 & -10\end{array}$ 


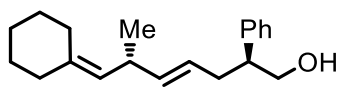

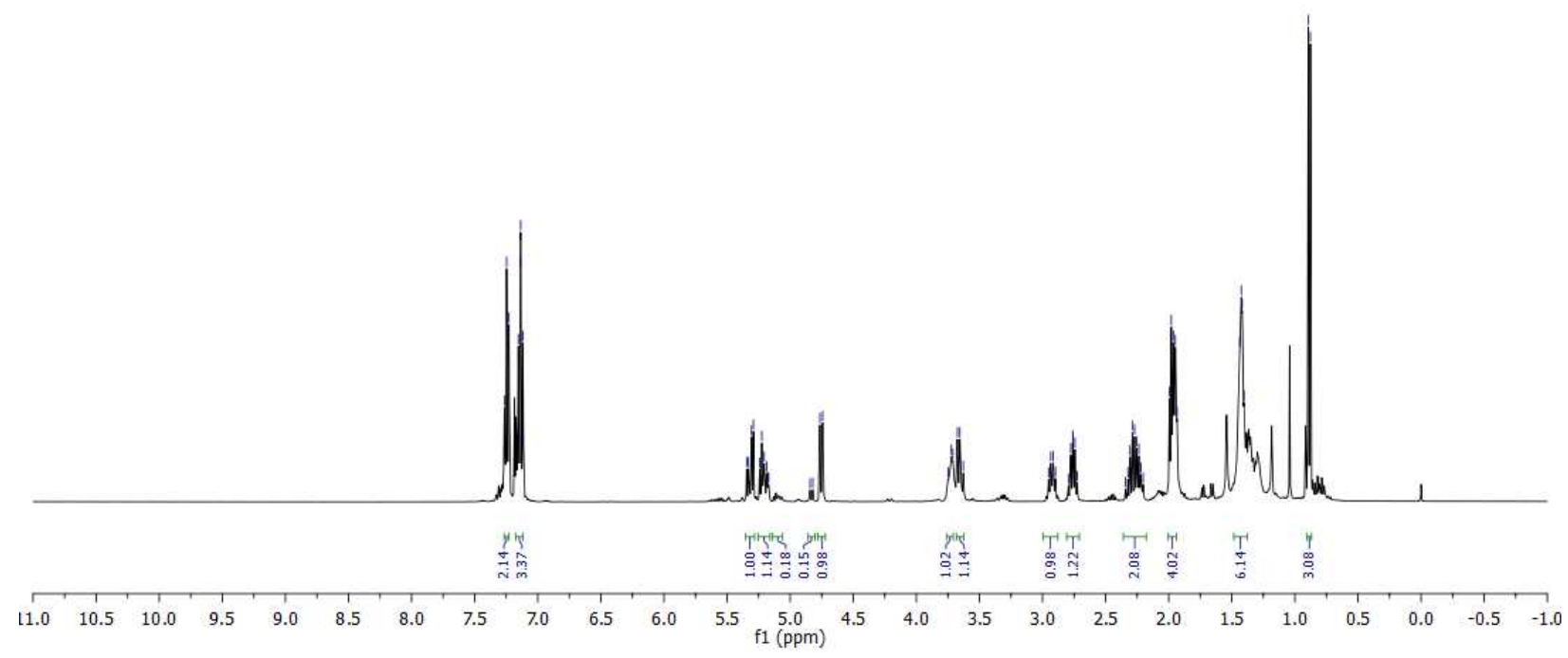

Anthony400-2019
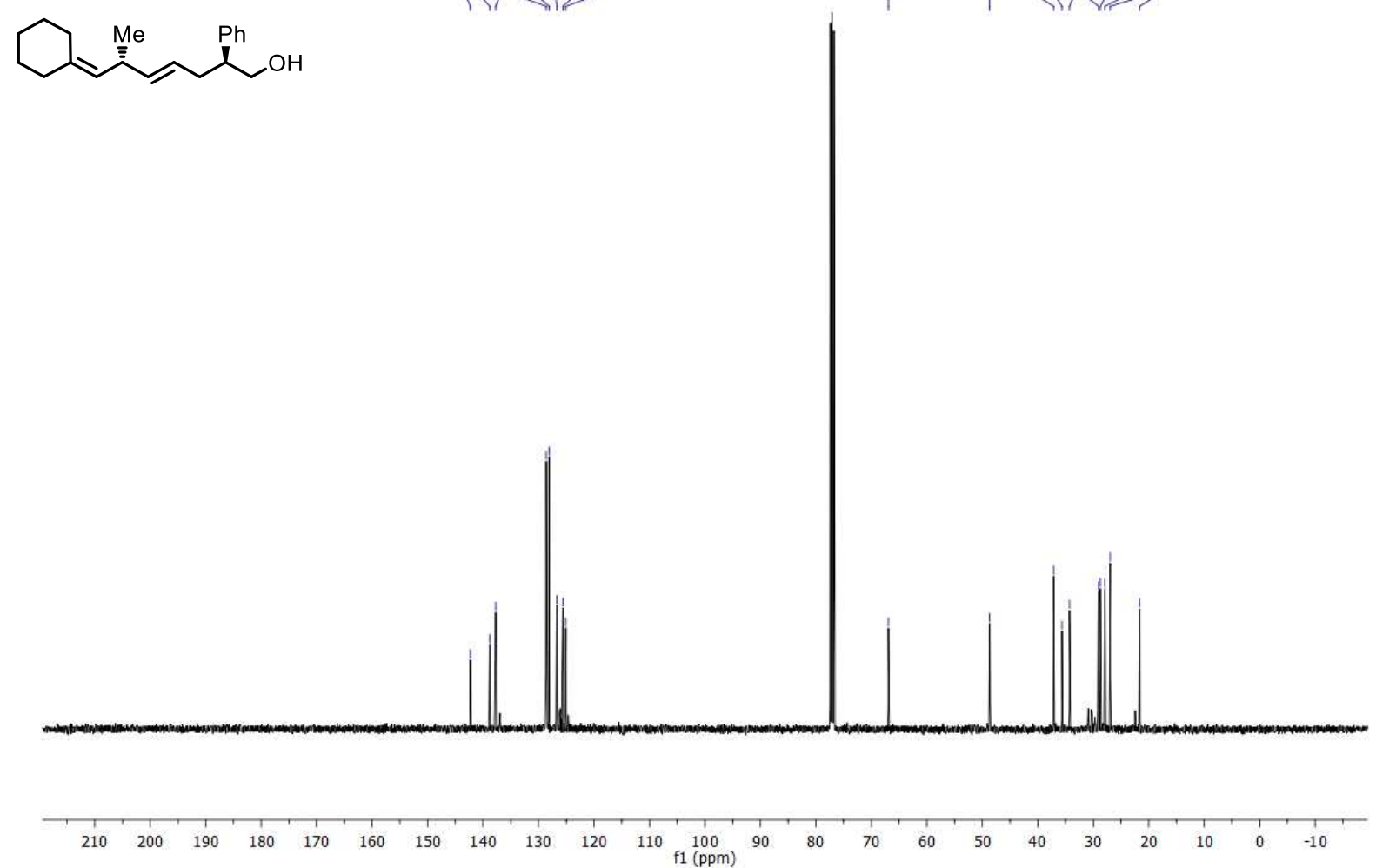


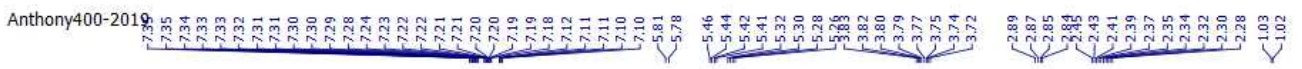<smiles>[Y6]C(C=C(c1ccccc1)c1ccccc1)/C=C/CC(CO)c1ccccc1</smiles>

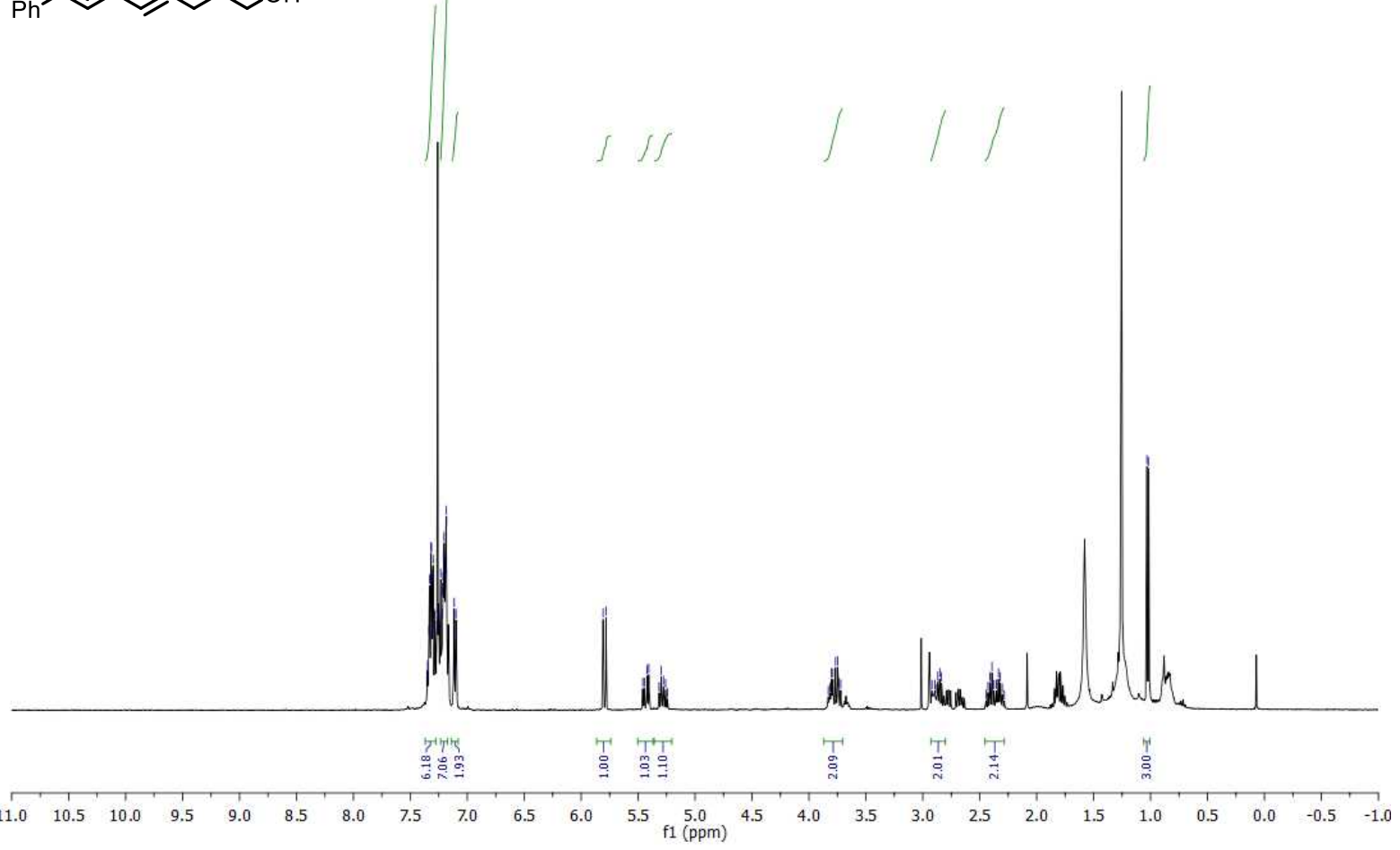

Anthony400-2019

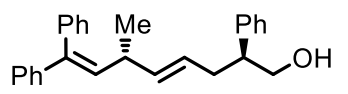

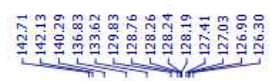
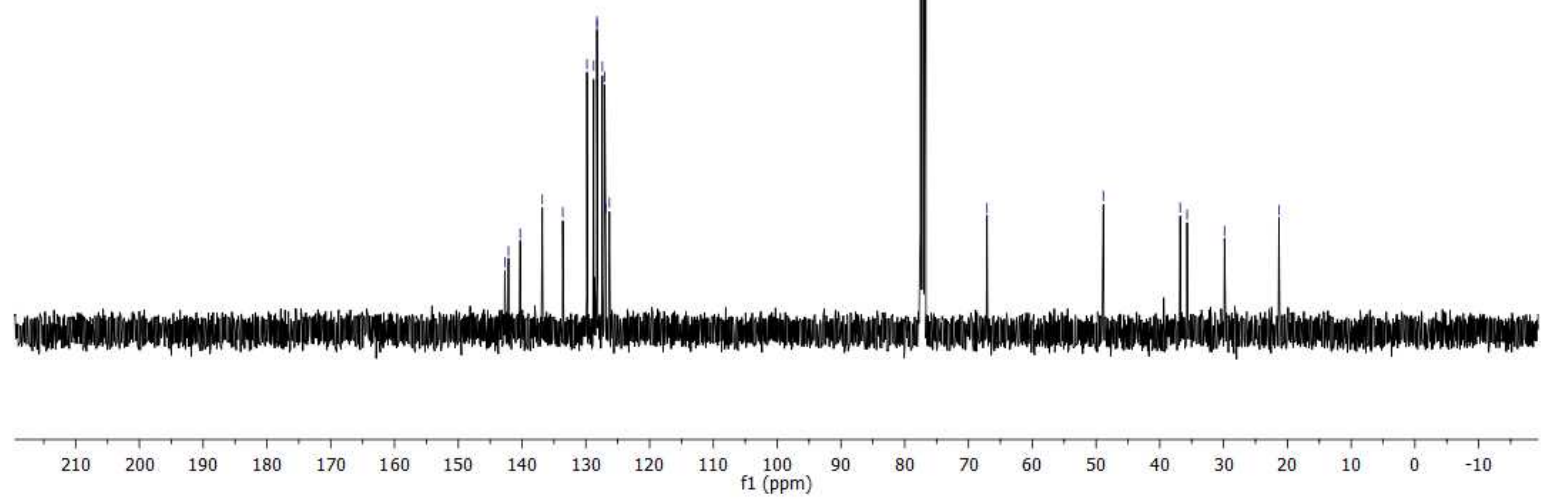

S67 


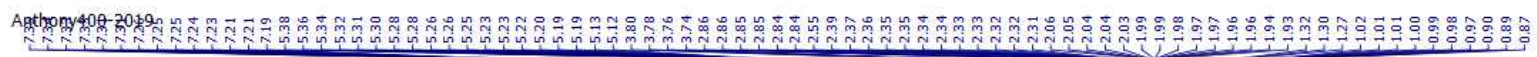
$\mathrm{C}_{7} \mathrm{H}_{15}$

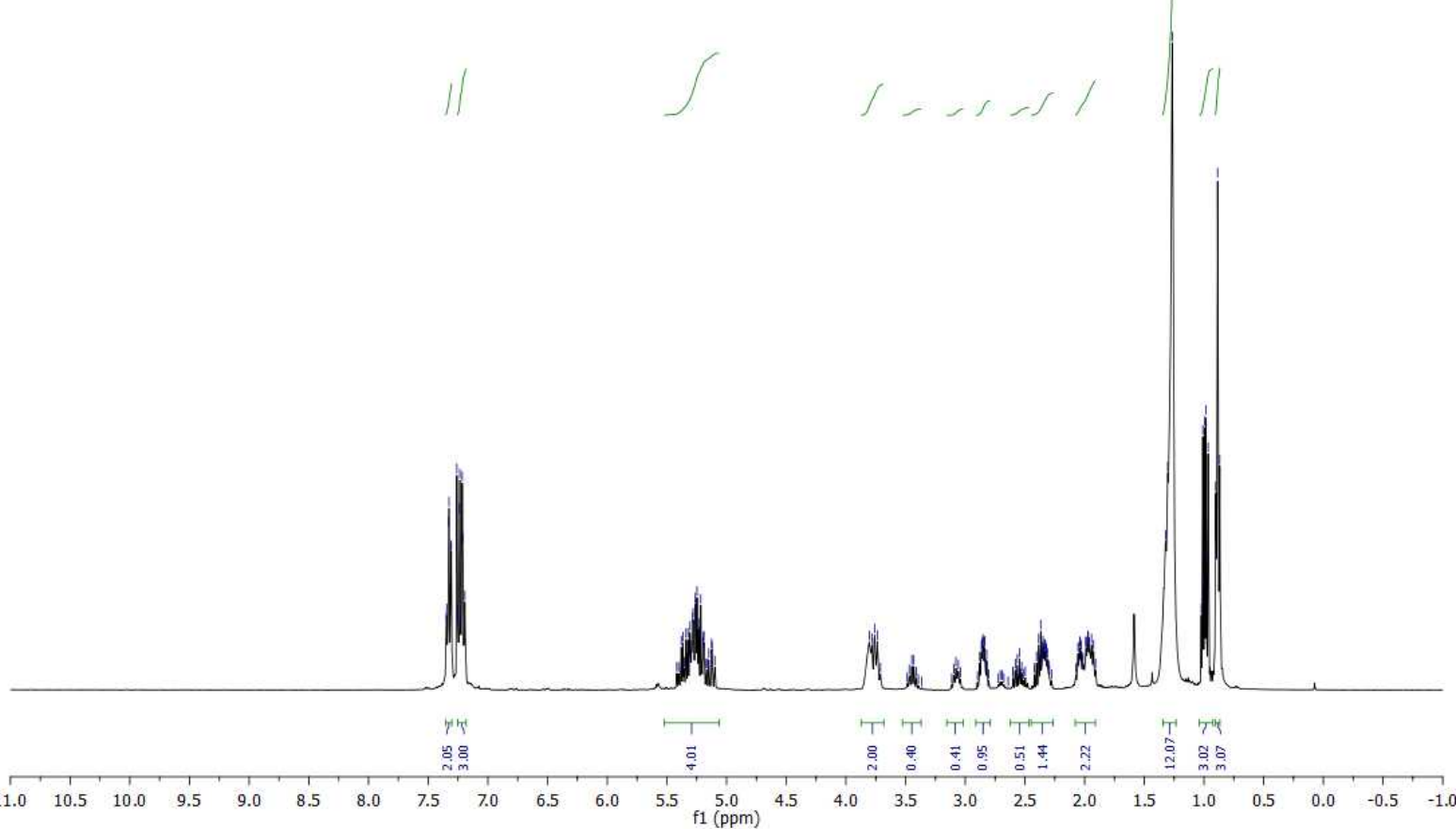

Anthony400-2019

$\mathrm{C}_{7} \mathrm{H}_{15}$
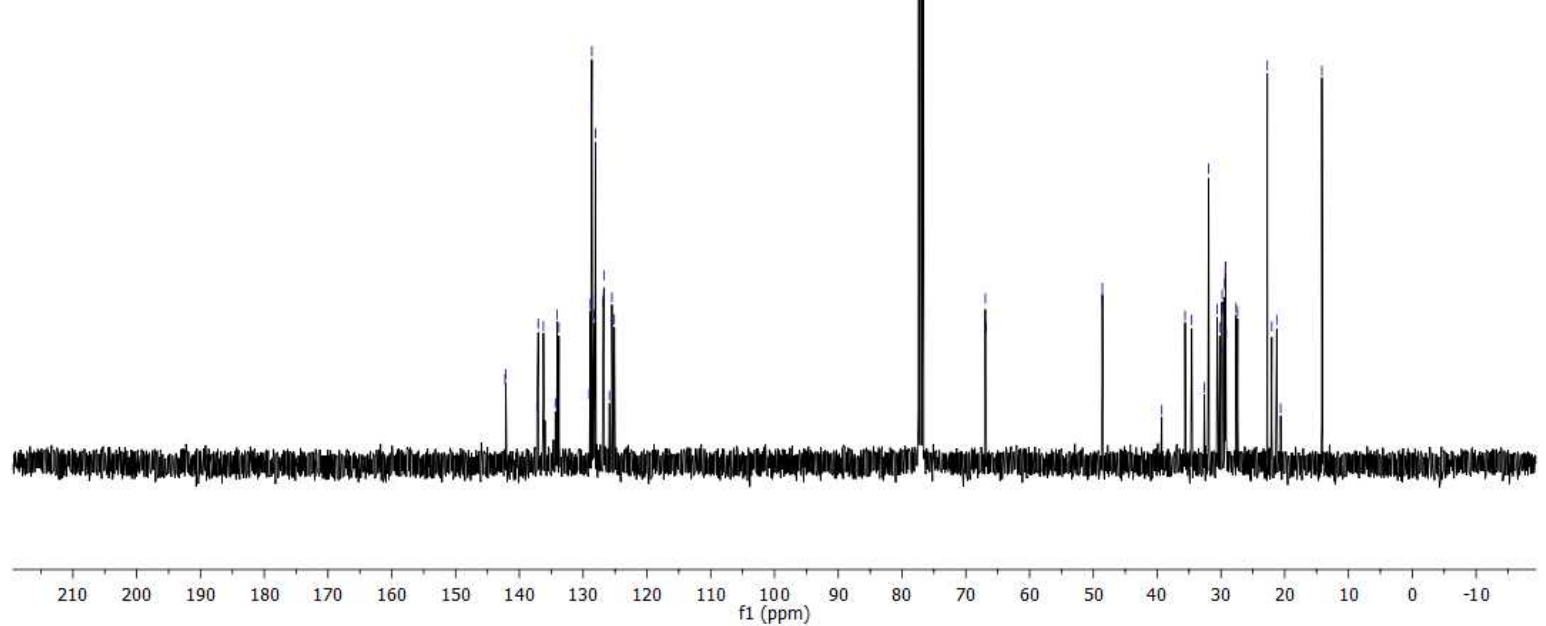


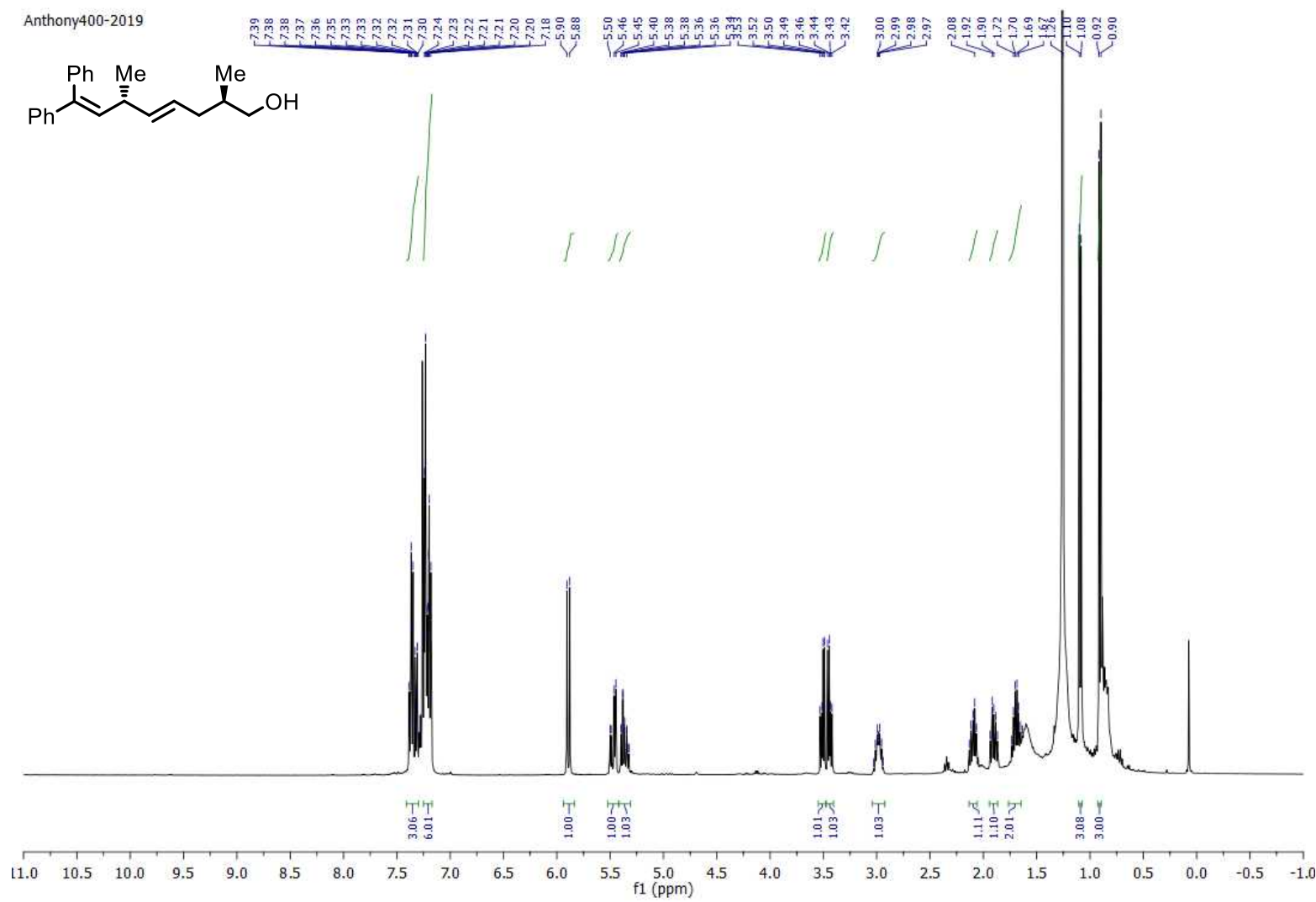

Anthony400-2019

事

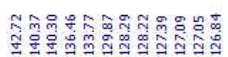

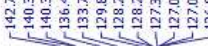

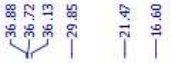
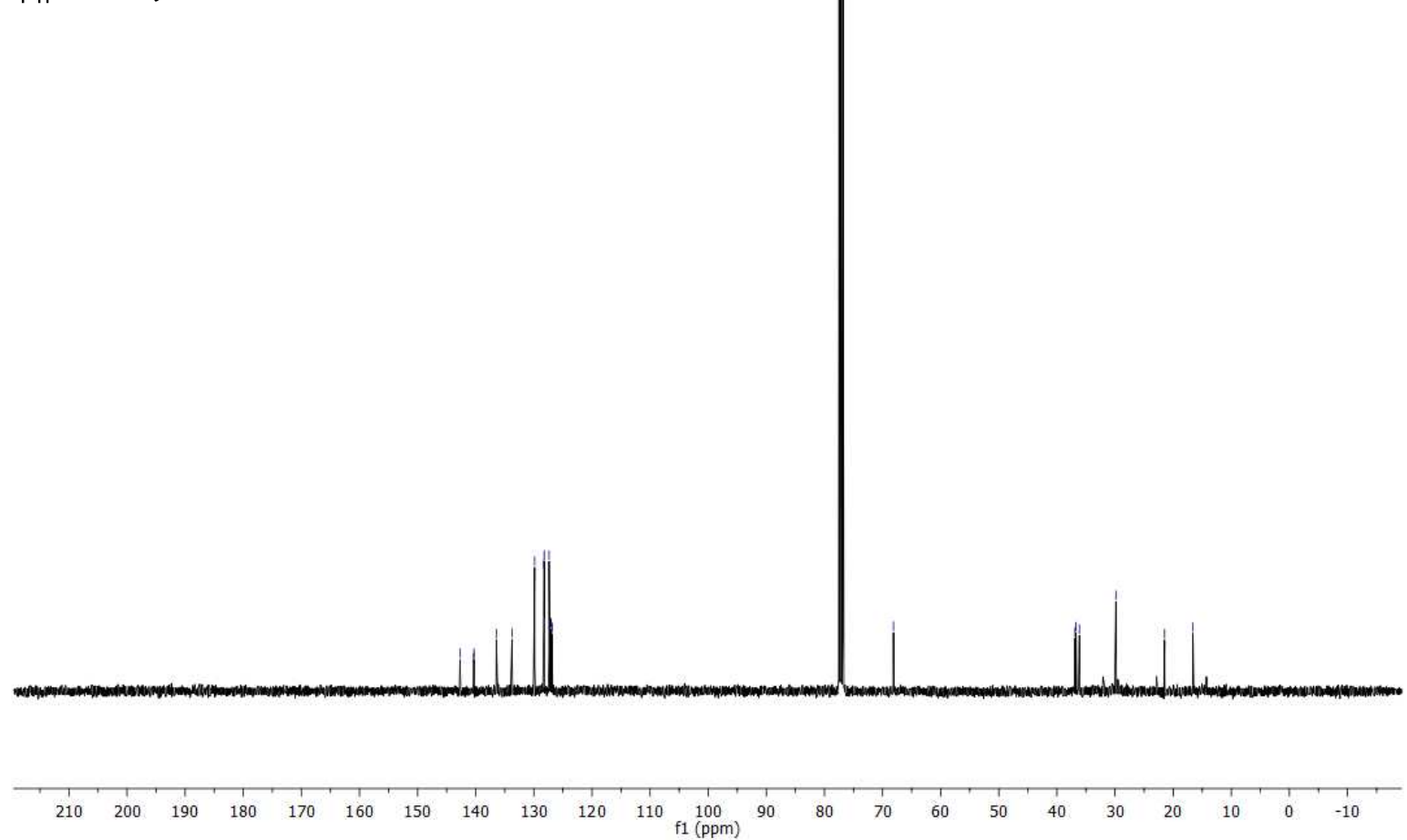

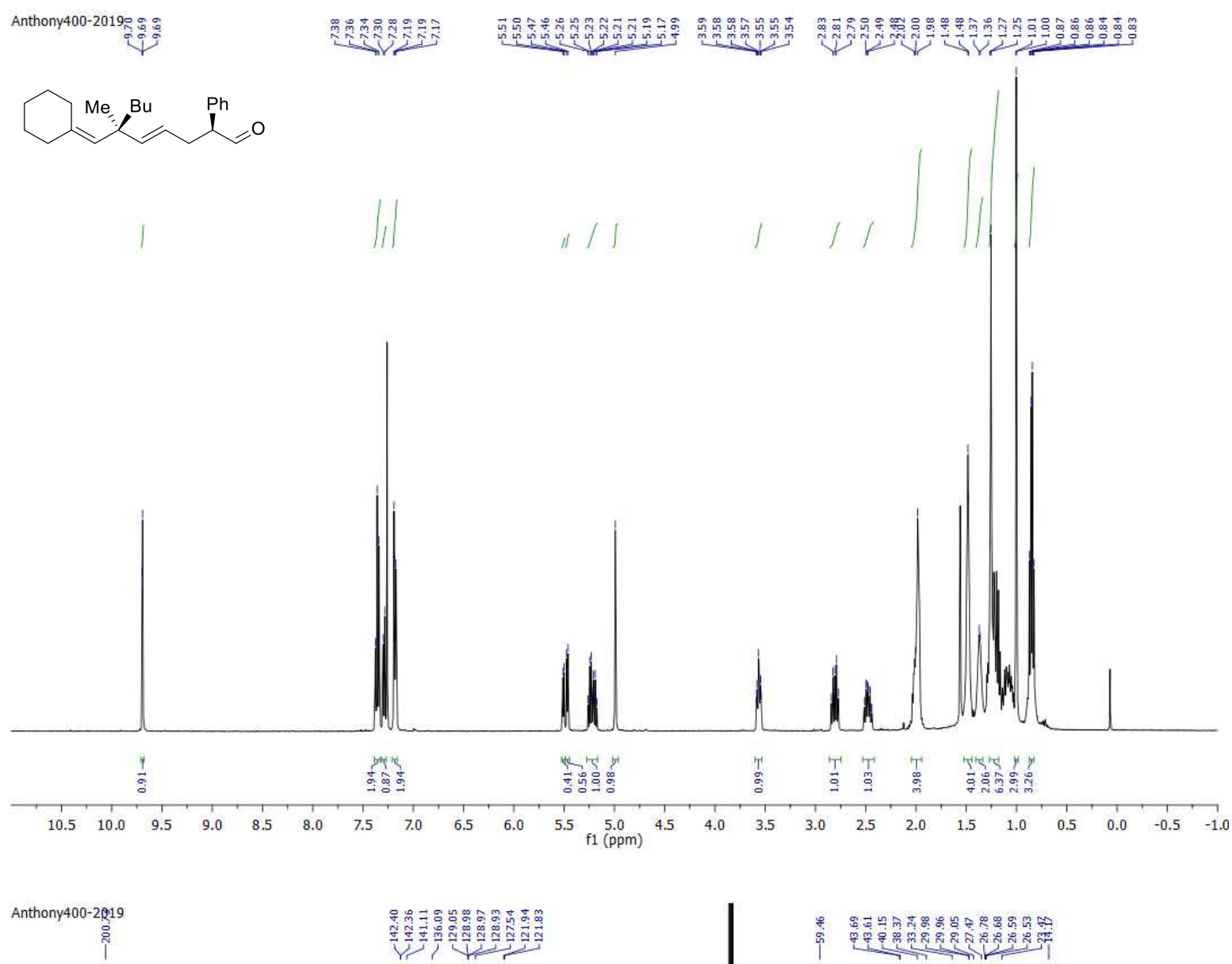

$\mathrm{O}^{\mathrm{Me}} \mathrm{B}^{\mathrm{Bu}}$
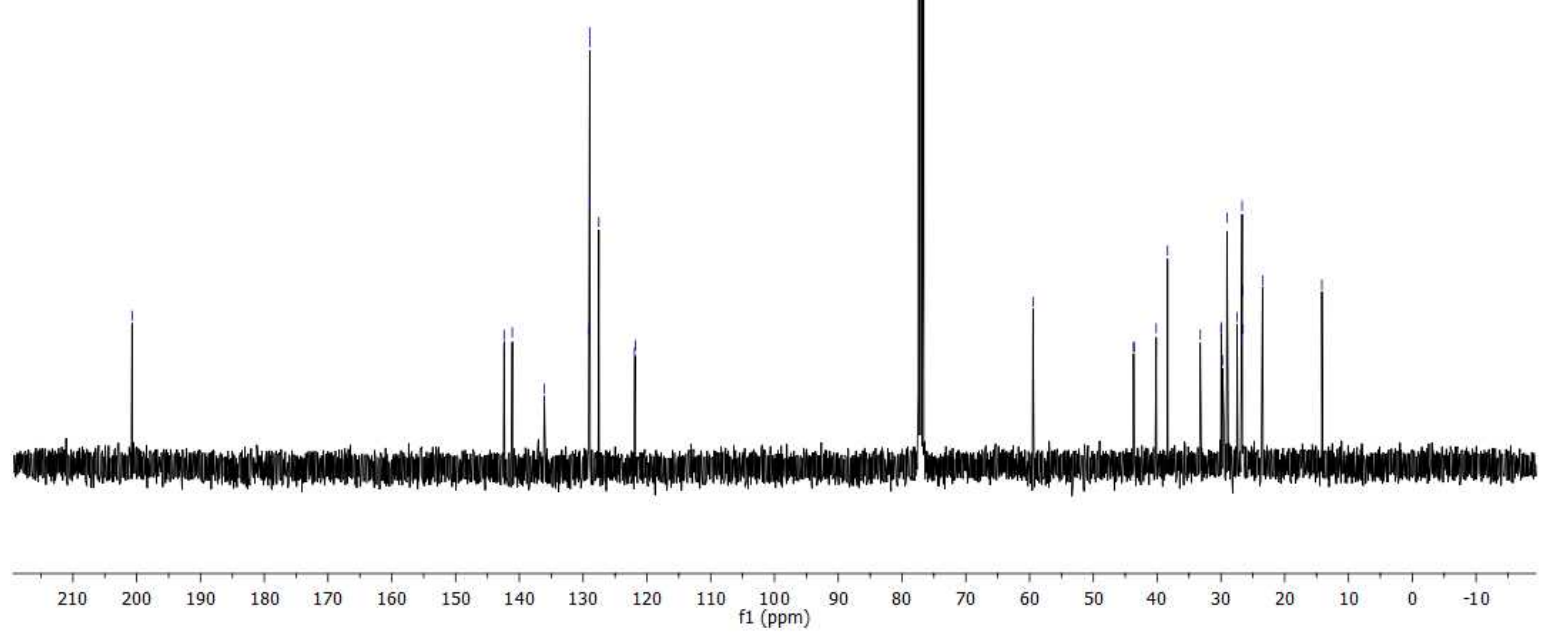


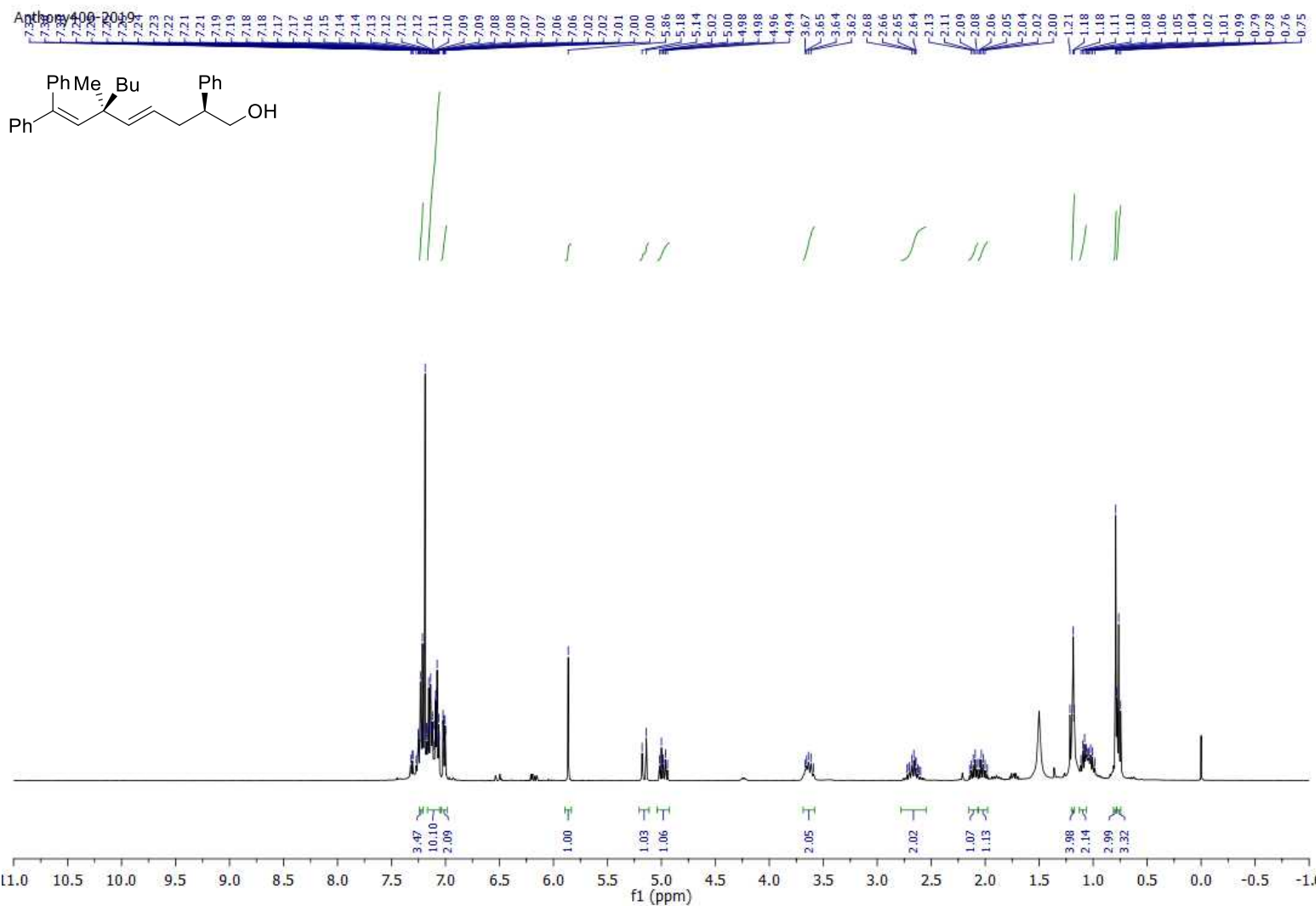

Anthony400-2019

PhMe
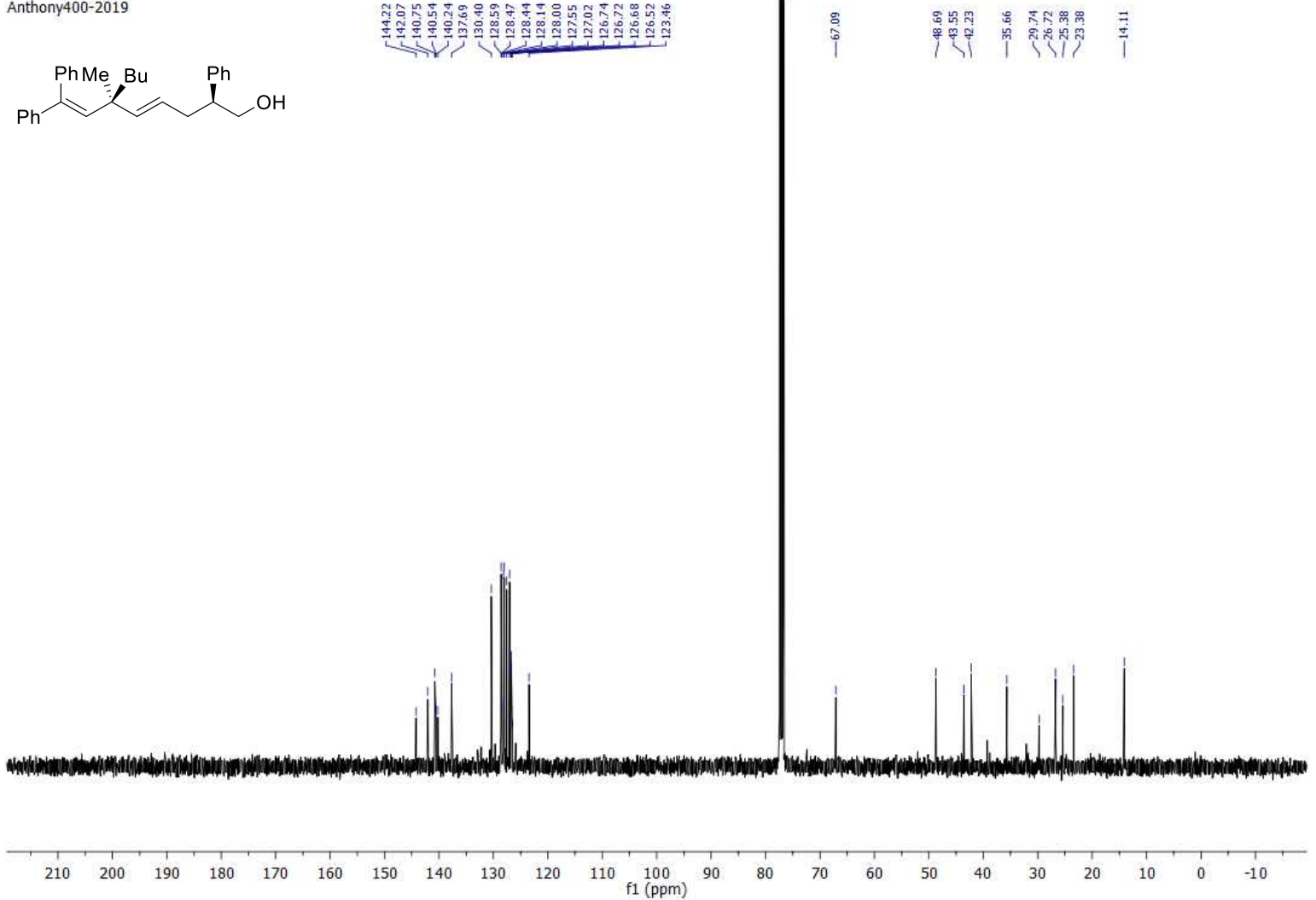


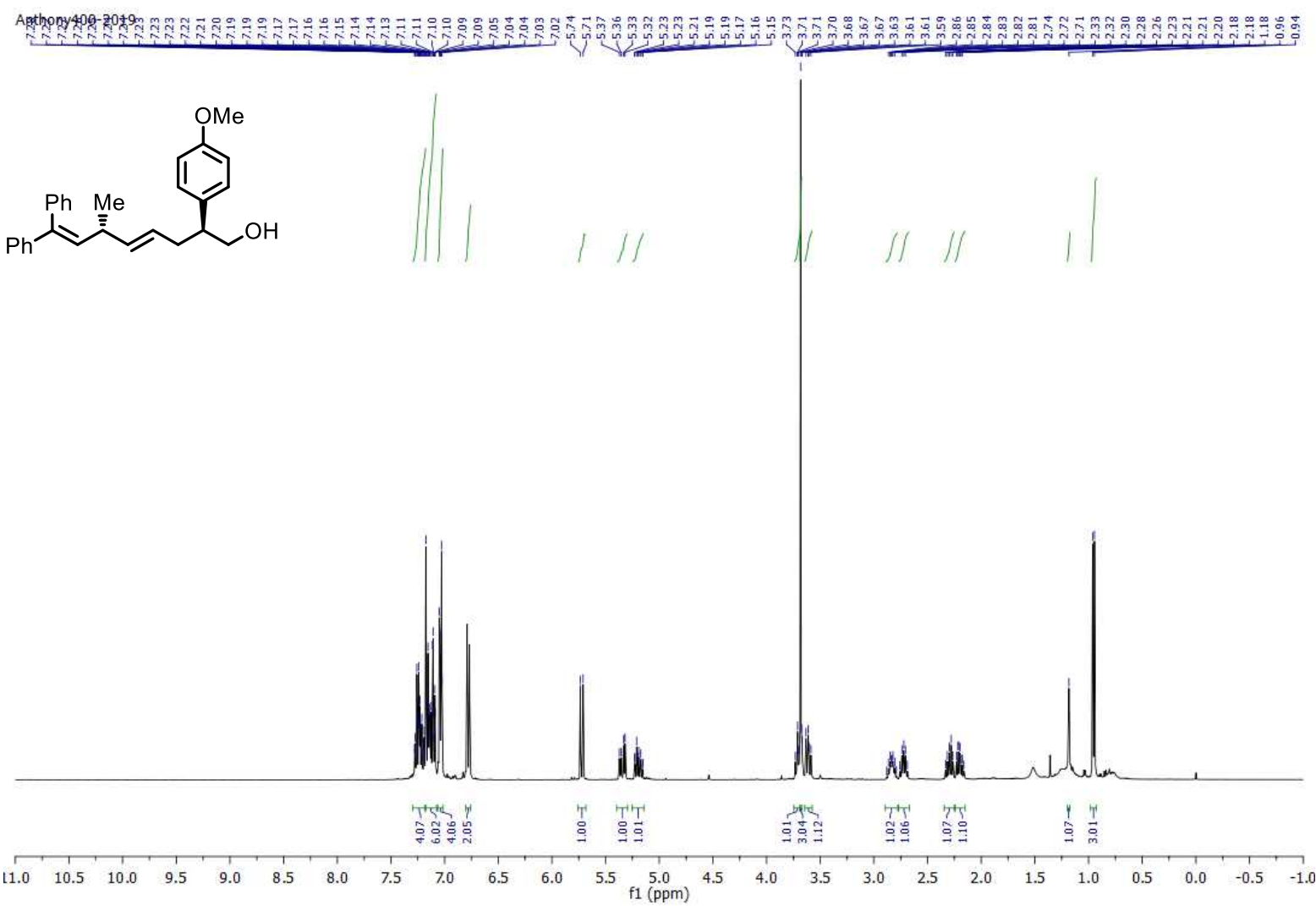

Anthony400-2019

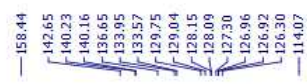

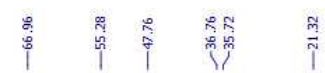
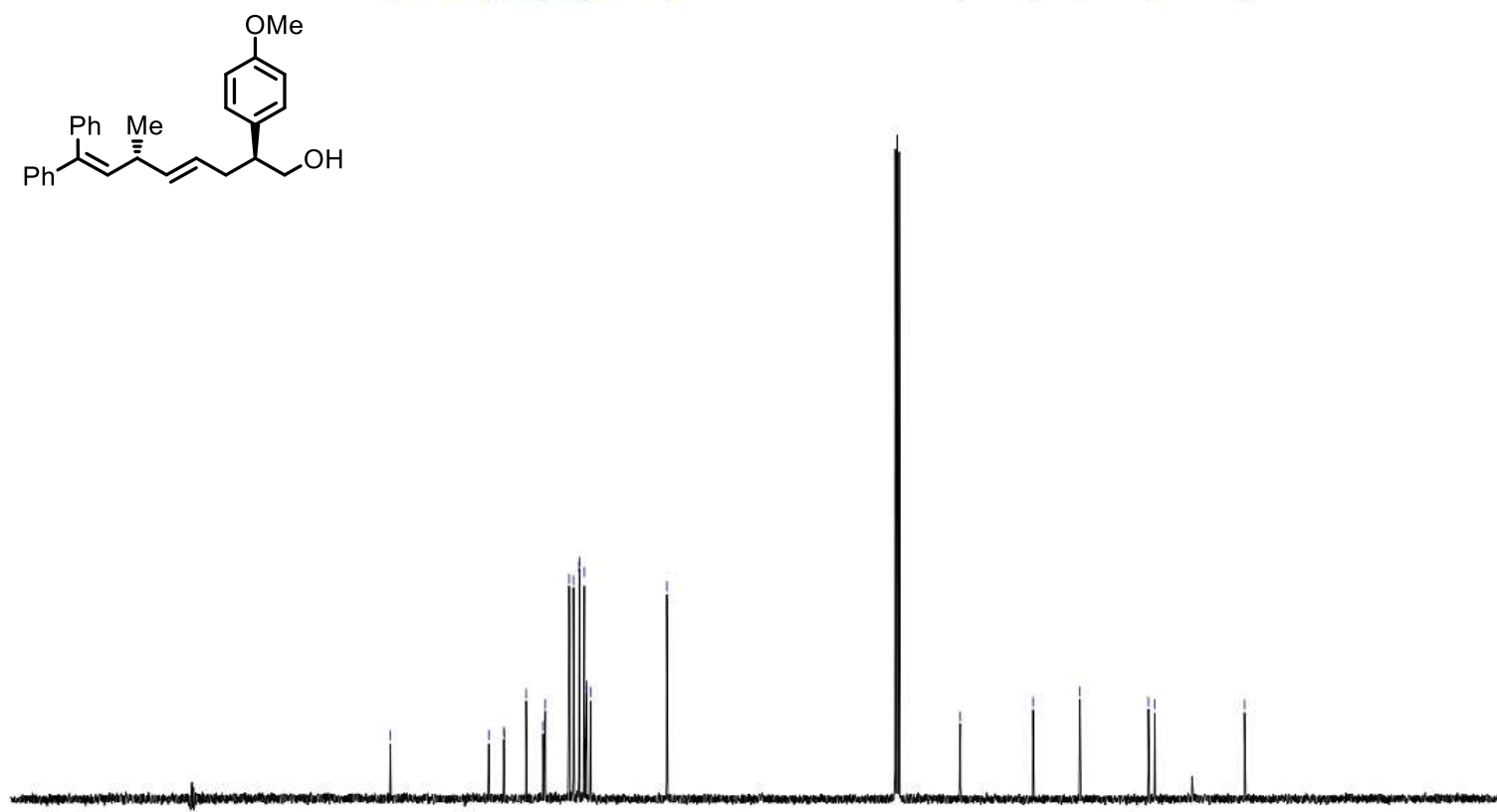

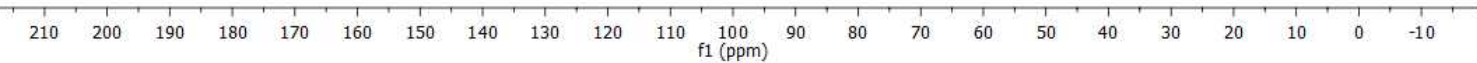




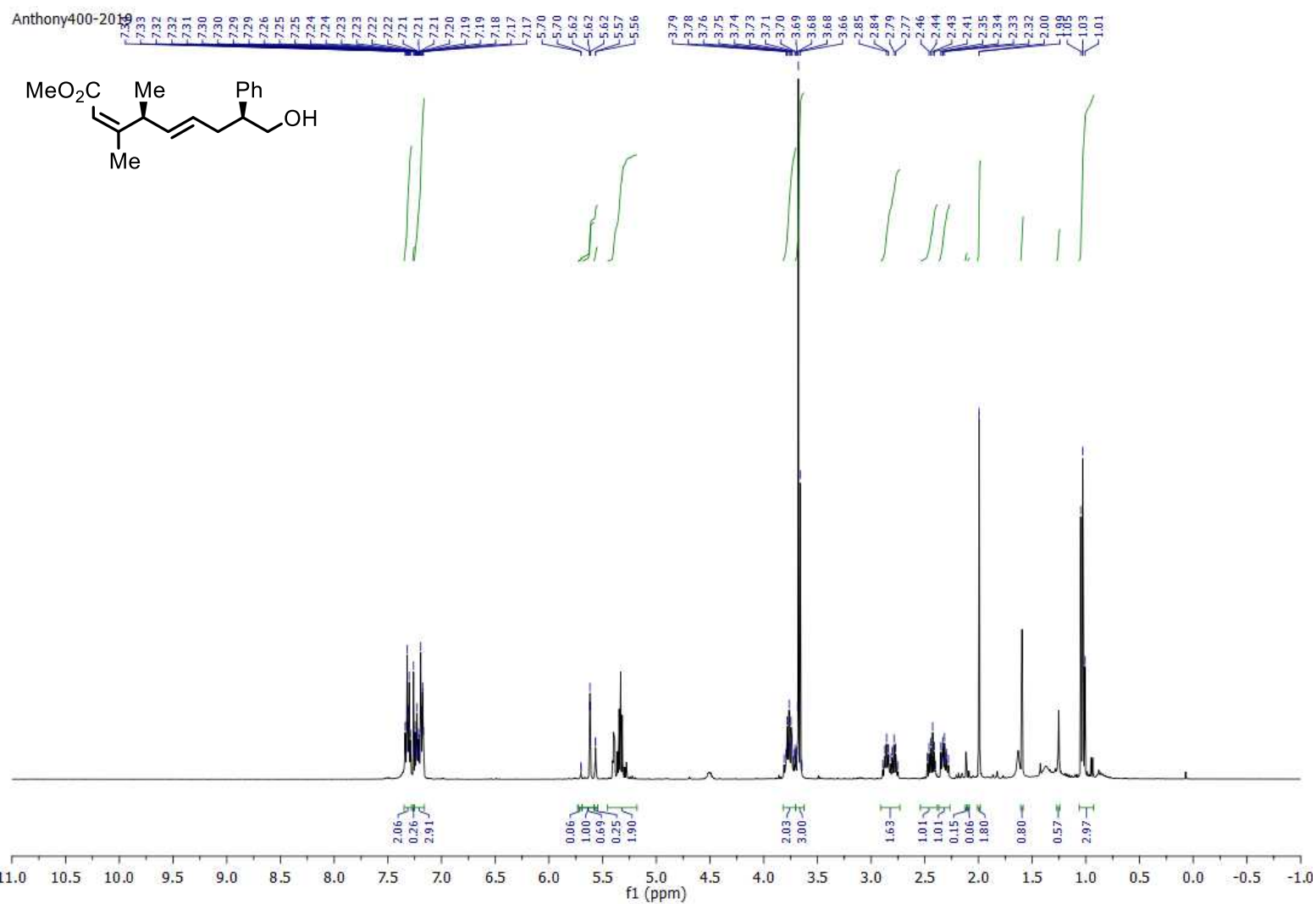

Anthony400-2019
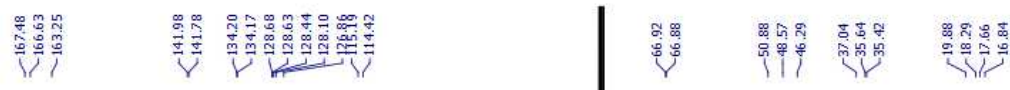

$\mathrm{MeO}_{2}$

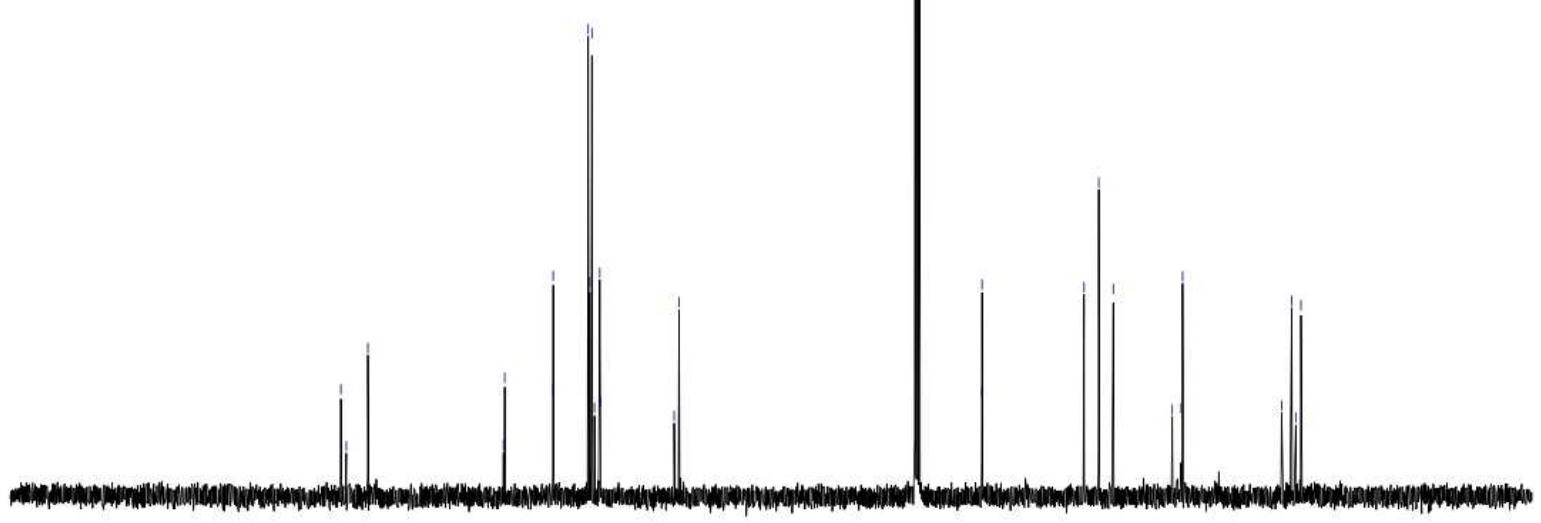

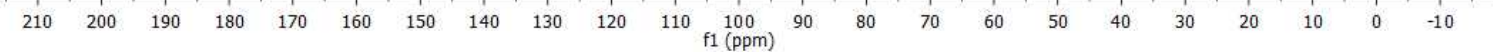




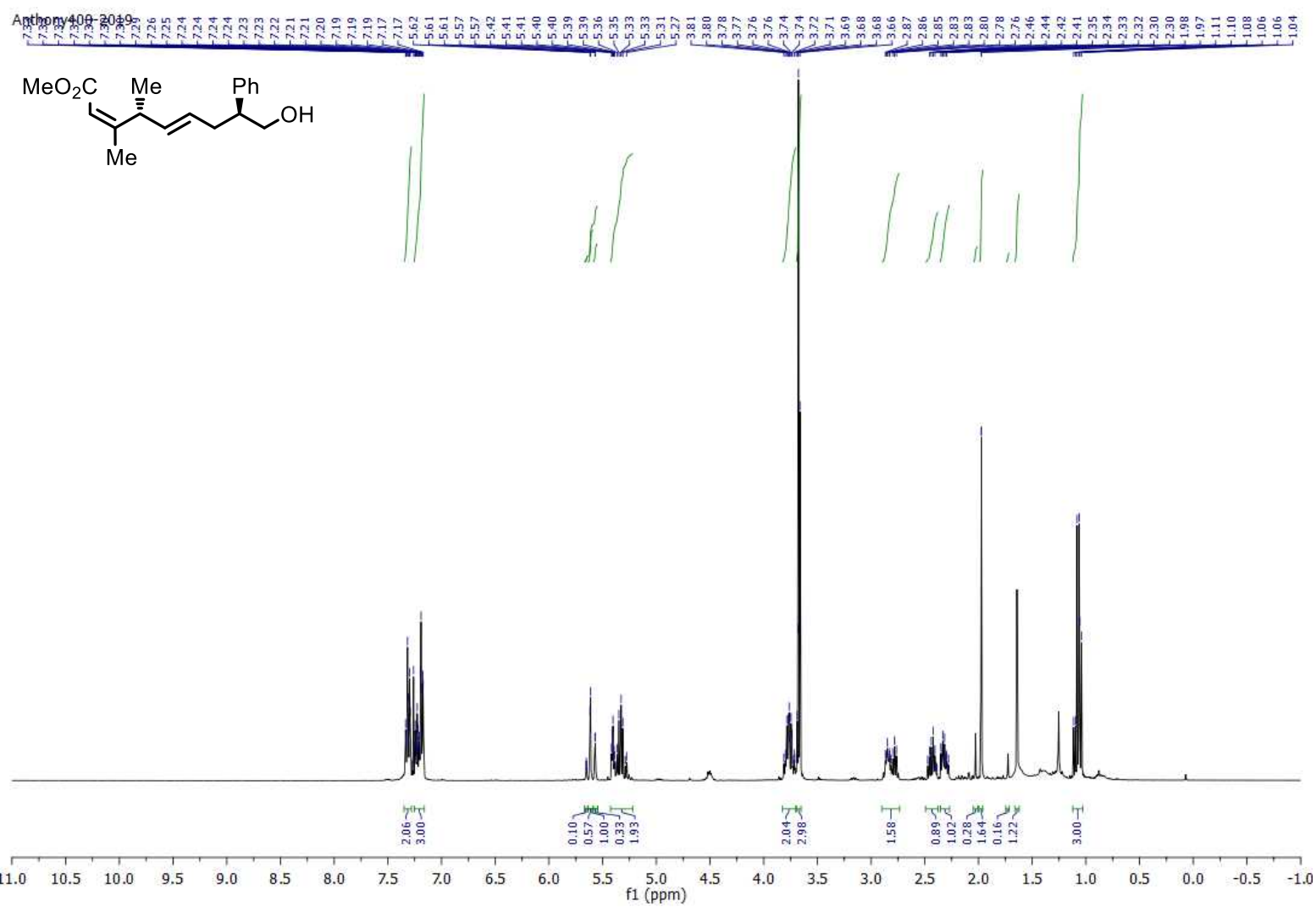

Anthony400-2019

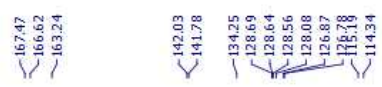

$\mathrm{MeO}_{\mathrm{Me}}^{\mathrm{Me}}$
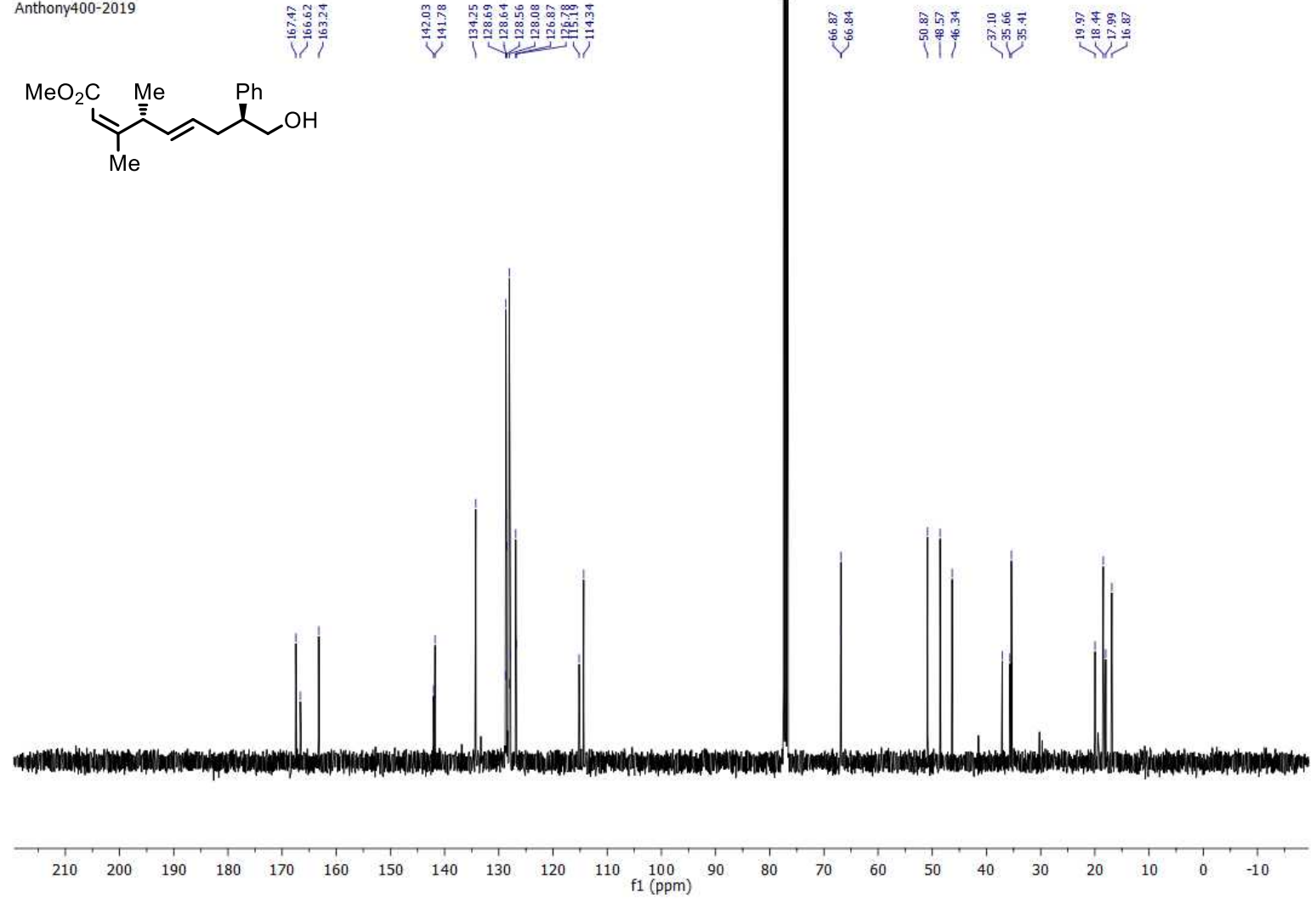


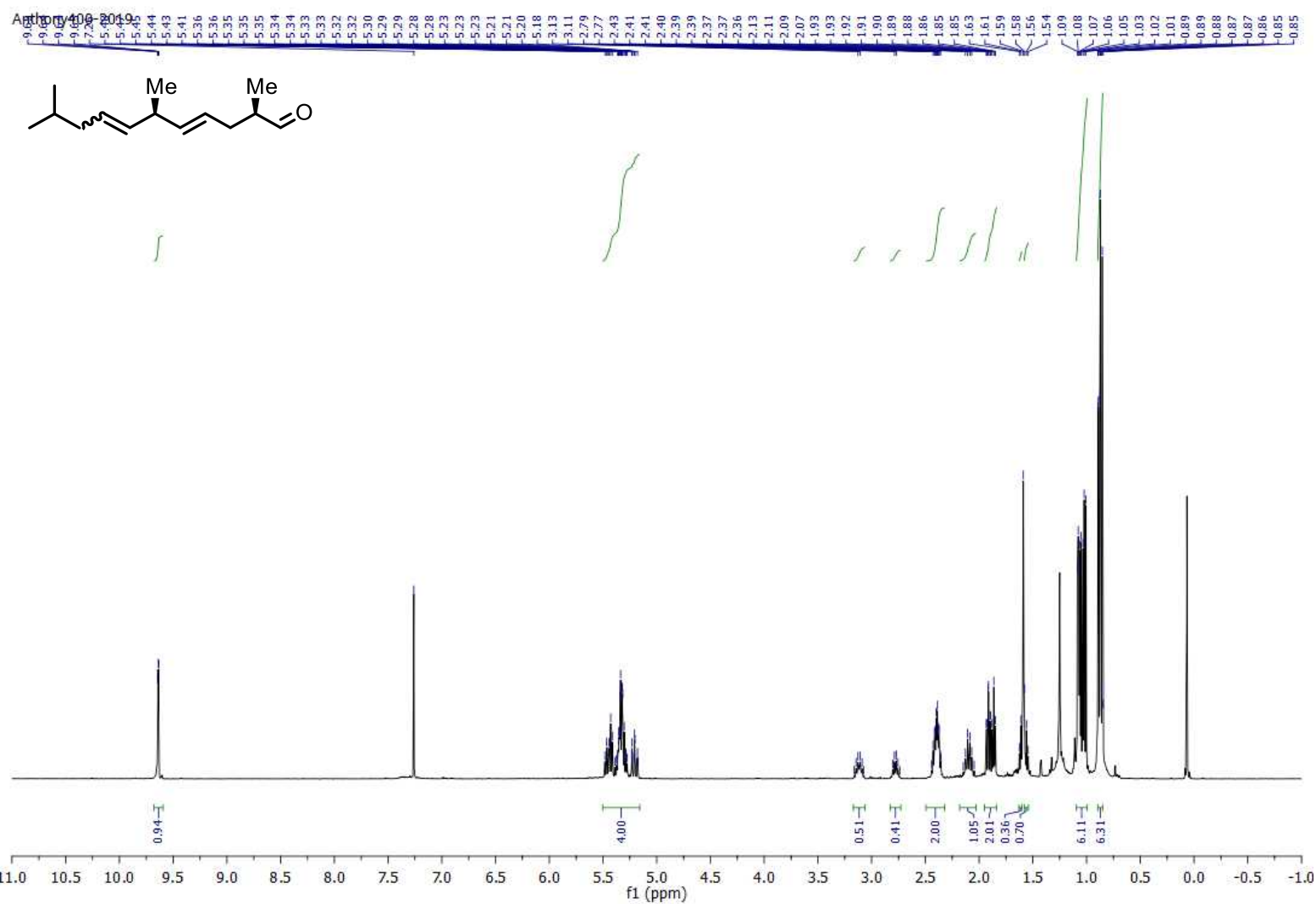

Anthony40g-2019

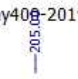

人 Me $\mathcal{M e}^{\text {Me }}$
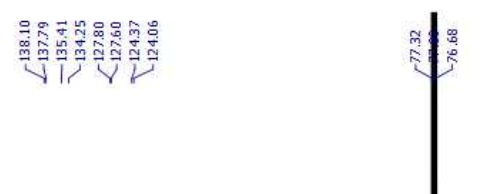

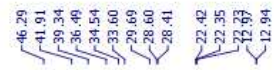

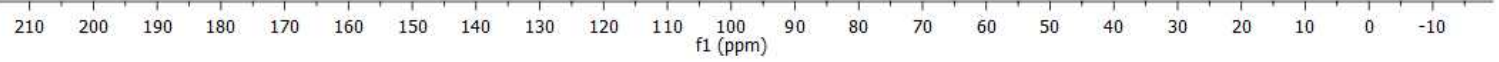



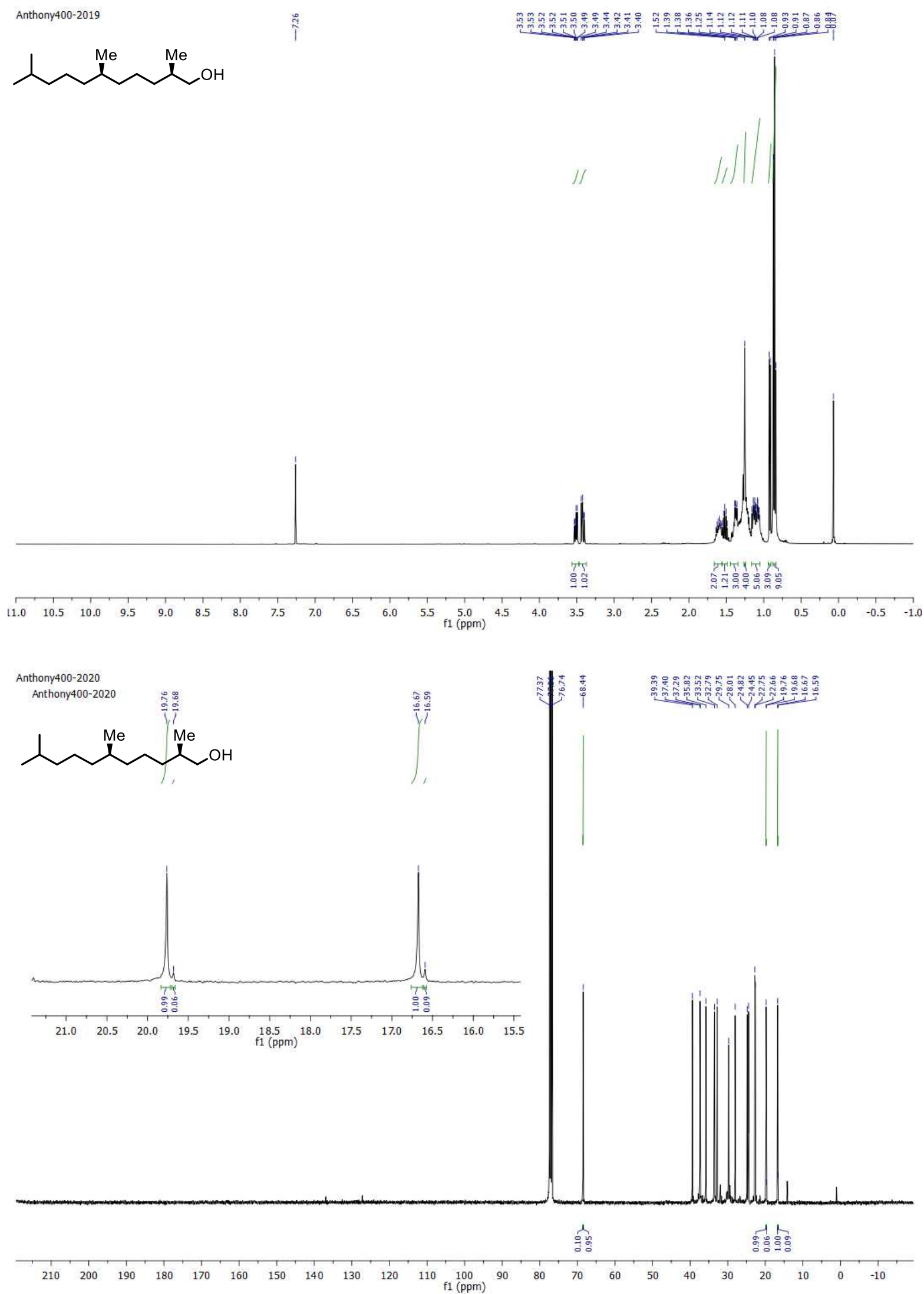